\title{
Sensitivity of ice sheet surface velocity and elevation to variations in basal friction and topography in the full Stokes and shallow-shelf approximation frameworks using adjoint equations
}

\author{
Gong Cheng ${ }^{1}$, Nina Kirchner ${ }^{2,3}$, and Per Lötstedt ${ }^{1}$ \\ ${ }^{1}$ Department of Information Technology, Uppsala University, P.O. Box 337, 75105 Uppsala, Sweden \\ ${ }^{2}$ Department of Physical Geography, Stockholm University, 10691 Stockholm, Sweden \\ ${ }^{3}$ Bolin Centre for Climate Research, Stockholm University, 10691 Stockholm, Sweden
}

Correspondence: Gong Cheng (cheng.gong@uci.edu)

Received: 16 April 2020 - Discussion started: 13 May 2020

Revised: 15 December 2020 - Accepted: 15 December 2020 - Published: 15 February 2021

\begin{abstract}
Predictions of future mass loss from ice sheets are afflicted with uncertainty, caused, among others, by insufficient understanding of spatiotemporally variable processes at the inaccessible base of ice sheets for which few direct observations exist and of which basal friction is a prime example. Here, we present a general numerical framework for studying the relationship between bed and surface properties of ice sheets and glaciers. Specifically, we use an inverse modeling approach and the associated time-dependent adjoint equations, derived in the framework of a full Stokes model and a shallow-shelf/shelfy-stream approximation model, respectively, to determine the sensitivity of grounded ice sheet surface velocities and elevation to time-dependent perturbations in basal friction and basal topography. Analytical and numerical examples are presented showing the importance of including the time-dependent kinematic free surface equation for the elevation and its adjoint, in particular for observations of the elevation. A closed form of the analytical solutions to the adjoint equations is given for a two-dimensional vertical ice in steady state under the shallow-shelf approximation. There is a delay in time between a seasonal perturbation at the ice base and the observation of the change in elevation. A perturbation at the base in the topography has a direct effect in space at the surface above the perturbation, and a perturbation in the friction is propagated directly to the surface in time.
\end{abstract}

\section{Introduction}

Over the last decades, ice sheets and glaciers have experienced mass loss due to global warming, both in the polar regions and also outside of Greenland and Antarctica (Farinotti et al., 2015; Mouginot et al., 2019; Pörtner et al., 2019; Rignot et al., 2019). The most common benchmark date for which future ice sheet and glacier mass loss and associated global mean sea level rise is predicted is the year $2100 \mathrm{CE}$, but recently, even the year $2300 \mathrm{CE}$ and beyond are considered (Pörtner et al., 2019; Steffen et al., 2018). Global mean sea level rise is predicted to continue well beyond $2100 \mathrm{CE}$ in the warming scenarios typically referred to as RCPs (representative concentration pathways; see van Vuuren et al., 2011), but rates and ranges are afflicted with uncertainty, caused by, among others, insufficient understanding of spatiotemporally variable processes at the inaccessible base of ice sheets and glaciers (Pörtner et al., 2019; Ritz et al., 2015). These include the geothermal heat regime, subglacial and base-proximal englacial hydrology, and particularly the sliding of the ice sheet and glaciers across their base, for which only few direct observations exist (Fisher et al., 2015; Key and Siegfried, 2017; Maier et al., 2019; Pattyn and Morlighem, 2020).

In computational models of ice dynamics, the description of sliding processes, including their parametrization, plays a central role and can be treated in two fundamentally different ways, viz. using a so-called forward approach on the one hand or an inverse approach on the other hand. In a forward approach, an equation referred to as a sliding law is derived 
from a conceptual friction model and provides a boundary condition to the equations describing the dynamics of ice flow (in glaciology often referred to as the full Stokes (FS) model), which, once solved, render, e.g., ice velocities part of the solution. Studies of frictional models and resulting sliding laws for glacier and ice sheet flow emerged in the 1950s see, e.g., Fowler (2011), Iken (1981), Lliboutry (1968), Nye (1969), Schoof (2005), and Weertman (1957) - have subsequently been implemented into numerical models of ice sheet and glacier behavior - e.g., in Brondex et al. (2017), Brondex et al. (2019), Gladstone et al. (2017), Tsai et al. (2015), Wilkens et al. (2015), and Yu et al. (2018) - and continue to be discussed (Zoet and Iverson, 2020), occasionally controversially (Minchew et al., 2019; Stearn and van der Veen, 2018).

Because few or no observational data are available to constrain the parameters in such sliding laws (Minchew et al., 2016; Sergienko and Hindmarsh, 2013), actual values of the former, and their variation over time (Jay-Allemand et al., 2011; Schoof, 2010; Sole et al., 2011; Vallot et al., 2017), often remain elusive. Yet, they can be obtained computationally by solving an inverse problem provided that observations of, e.g., ice velocities at the ice surface and elevation of the ice surface are available (Gillet-Chaulet et al., 2016; Isaac et al., 2015). Note that the same approach, here described for the case of the sliding law, can be used to determine other "inaccessibles", such as optimal initial conditions for ice sheet modeling (Perego et al., 2014), the sensitivity of melt rates beneath ice shelves in response to ocean circulation (Heimbach and Losch, 2012), the geothermal heat flux at the ice base (Zhu et al., 2016), or to estimate basal topography beneath an ice sheet (Monnier and des Boscs, 2017; van Pelt et al., 2013). The latter is not only difficult to separate from the determination of the sliding properties (KyrkeSmith et al., 2018; Thorsteinsson et al., 2003), but also has limitations related to the spatial resolution of surface data and/or measurement errors; see Gudmundsson $(2003,2008)$ and Gudmundsson and Raymond (2008).

Adopting an inverse approach, the strategy is to minimize an objective function describing the deviation of observed target quantities (such as the ice velocity) from their counterparts as predicted following a forward approach when a selected parameter in the forward model (such as the friction parameter in the sliding law) is varied. The gradient of the objective function is computed by solving the so-called adjoint equations to the forward equations, where the latter often are slightly simplified, such as by assuming a constant ice thickness or a constant viscosity (MacAyeal, 1993; Petra et al., 2012). However, when inferring friction parameter(s) in a sliding law using an inverse approach, recent work (Goldberg et al., 2015; Jay-Allemand et al., 2011) has shown that it is not sufficient to consider the time-independent (steadystate) adjoint to the momentum balance in the FS model. Rather, it is necessary to include the time-dependent advection equation for the ice surface elevation in the inversion.
Likewise, but perhaps more intuitively understandable, the choice of the underlying glaciological model (FS model vs., e.g., shallow-shelf/stream approximation (SSA) model; see Sect. 2) has an impact on the values of the friction parameters obtained from the solution of the corresponding inverse problem (Gudmundsson, 2008; Schannwell et al., 2019). A related problem is to find the relation between perturbations of the friction parameters and the topography at the base and the resulting perturbations of the ice velocity and the elevation at the surface. The parameter sensitivity of the solutions is studied in Gudmundsson (2008) and Gudmundsson and Raymond (2008) by linearizing the governing equations. They show that basal perturbations of short wavelength are damped when they reach the surface, but the effect of long wavelengths can be observed at the top.

Here, we present an analysis of the sensitivity of the velocity field and the elevation of the surface of a dynamic, grounded ice sheet (modeled by both FS and SSA, briefly described in Sect. 2) to perturbations in the sliding parameters contained in Weertman's law (Weertman, 1957) and the topography at the ice base. The perturbations in a velocity component or the ice surface elevation at a certain location and time are determined by the solutions to the forward equations and the associated adjoint equations of a grounded ice sheet. A certain type of perturbation at the base may cause a very small perturbation at the top of the ice. Such a basal perturbation will be difficult to infer from surface observations in an inverse problem. High-frequency perturbations in space and time are examples when little is propagated to the surface. This is also the conclusion in Gudmundsson and Raymond (2008), derived from an analysis differing from the one presented here. The adjoint problem that is solved here to determine this sensitivity (Sect. 3) goes beyond similar earlier works by MacAyeal (1993) and Petra et al. (2012) because it includes the time-dependent advection equation for the kinematic free surface. The key concepts and steps introduced in Sect. 3 are supplemented by detailed derivations, collected in Appendix A. The same adjoint equations are applicable in the inverse problem to compute the gradient of the objective function and to quantify the uncertainty in the surface velocity and elevation due to uncertainties at the ice base. Examples of uncertainties are measurement errors in the basal topography and unknown variations in the parameters in the friction model. Analytical solutions in two dimensions of the stationary adjoint equations subjected to simplifying assumptions are presented, from which the dependence of the parameters, on, e.g., friction coefficients and bedrock topography, becomes obvious. The time-dependent adjoint equations are solved numerically, and the sensitivity to perturbations varying in time is investigated and illustrated.

The sensitivity of the surface velocity and elevation to perturbations in the friction and topography is quantified in extensive numerical computations in a companion paper (Cheng and Lötstedt, 2020). The adjoint equations derived and studied analytically in this paper are solved numerically 
for the FS and SSA models in Cheng and Lötstedt (2020) and compared to direct calculations of the surface perturbations with the forward equations. Discrete transfer functions are computed and analyzed as in Gudmundsson (2008) for the relation between surface perturbations and basal perturbations. While Gudmundsson's analysis is based on Fourier analysis, the analysis in Cheng and Lötstedt (2020) relies on analytical solutions of the SSA equations. The analysis in this paper and the numerical experiments in Cheng and Lötstedt (2020) confirm the conclusion in Gudmundsson (2008) that the perturbations with a long wavelength and low frequency will propagate to the surface while those of a short wavelength and high frequency are damped.

\section{Ice models}

In this section, the equations emerging from adopting a forward approach to describing ice dynamics are presented, together with relevant boundary conditions, for the FS (4) and SSA model (9), respectively. These, and the notation and terminology introduced here, provide the framework in which the adjoint equations are discussed in Sect. 3.

The flow of large bodies of ice is described with the help of the conservation laws of mass, momentum, and energy (Greve and Blatter, 2009), which together pose a system of nonlinear partial differential equations (PDEs) commonly referred to as the FS equations in glaciological applications. In the FS equations, nonlinearity is introduced through the viscosity in Glen's flow law, a constitutive relation between strain rates and stresses (Glen, 1955). Continental sized ice masses (ice sheets and, if applicable, their floating extensions known as ice shelves) are shallow in the sense that their vertical extension $V$ is orders of magnitudes smaller than their horizontal extension $L$, such that the aspect ratio $V / L$ is on the order $10^{-2}$ to $10^{-3}$. The aspect ratio is used to introduce simplifications to the FS equations, resulting, e.g., in the shallow-ice (SIA) (Hutter, 1983), shallowshelf (Morland, 1987), and shelfy-stream (MacAyeal, 1989) approximations, parts of which can be coupled to FS using ISCAL (Ahlkrona et al., 2016), a method applicable to any FS framework and implemented in Elmer/Ice. They are all characterized by substantially reduced computational costs for numerical simulation, compared to using the FS model. A common simplification, also adopted in our analysis in the following, is the assumption of isothermal conditions, which implies that the balance of energy need not be considered.

The upper surface of the ice mass and also the ice-ocean interface constitute a moving boundary and satisfy an advection equation describing the evolution of its elevation and location (in response to mass gain, mass loss, or/and mass advection). For ice masses resting on bedrock or sediments, sliding needs to be parameterized at the interface. The interface between floating ice shelves and sea water in the ice shelf cavities is usually regarded as frictionless.

\subsection{Full Stokes model}

We adopt standard notation and denote vectors in bold italics and matrices in three-dimensional space in bold, and we denote derivatives with respect to the spatial coordinates and time by subscripts $x, y, z$, and $t$. The horizontal plane is spanned by the $x$ and $y$ coordinates, and $z$ is the coordinate in the vertical direction (see Fig. 1a). Specifically, we denote by $u_{1}, u_{2}$, and $u_{3}$ the velocity components of $\boldsymbol{u}=\left(u_{1}, u_{2}, u_{3}\right)^{\mathrm{T}}$ in the $x, y$, and $z$ direction, where $\boldsymbol{x}=(x, y, z)^{\mathrm{T}}$ is the position vector and $\mathrm{T}$ denotes the transpose. Further, the elevation of the upper ice surface is denoted by $h(x, y, t)$, the elevation of the bedrock and the location of the base of the ice are $b(x, y)$ and $z_{b}(x, y, t)$, and the ice thickness is $H=h-z_{b}$. Upstream of the grounding line, $\gamma_{\mathrm{GL}}, z_{b}=b$, and downstream of $\gamma_{\mathrm{GL}}$ we have $z_{b}>b$ (see Fig. 1). In two dimensions, $\gamma_{\mathrm{GL}}$ consists of one point with $x$ coordinate $x_{\mathrm{GL}}$.

The boundary $\Gamma$ enclosing the domain $\Omega$ occupied by the ice has different parts (see Fig. 1b). The lower boundaries of $\Omega$ are denoted by $\Gamma_{\mathrm{b}}$ (where the ice is grounded at bedrock) and $\Gamma_{\mathrm{w}}$ (where the ice has lifted from the bedrock and is floating on the ocean). These two regions are separated by the grounding line $\gamma_{\mathrm{GL}}$, defined by $\left(x_{\mathrm{GL}}(y), y\right)$ based on the assumption that ice flow is mainly along the $x$ axis. The upper boundary is denoted by $\Gamma_{\mathrm{S}}$ (ice surface) at $h(x, y, t)$ in Fig. 1a. The footprint (or projection) of $\Omega$ in the horizontal plane is denoted by $\omega$ and its boundary is $\gamma$.

The vertical lateral boundary (in the $x-z$ plane, Fig. 1b) has an upstream part denoted by $\Gamma_{\mathrm{u}}$ in black and a downstream part denoted by $\Gamma_{\mathrm{d}}$ in blue, where $\Gamma=\Gamma_{\mathrm{u}} \cup \Gamma_{\mathrm{d}}$. Obviously, if $\boldsymbol{x} \in \Gamma_{\mathrm{u}}$, then $(x, y) \in \gamma_{\mathrm{u}}$, or if $\boldsymbol{x} \in \Gamma_{\mathrm{d}}$, then $(x, y) \in$ $\gamma_{\mathrm{d}}$, where $\gamma=\gamma_{\mathrm{u}} \cup \gamma_{\mathrm{d}}$. Letting $\boldsymbol{n}$ be the outward-pointing normal on $\Gamma$ (or $\gamma$ in two dimensions $(x, y)$ ), the nature of ice flow renders the conditions $\boldsymbol{n} \cdot \boldsymbol{u} \leq 0$ at $\Gamma_{\mathrm{u}}$ and $\boldsymbol{n} \cdot \boldsymbol{u}>0$ at $\Gamma_{\mathrm{d}}$. For a two-dimensional flow line geometry (Fig. 1a), this corresponds to $\boldsymbol{x}=(x, z)^{\mathrm{T}}, \omega=[0, L], \gamma_{\mathrm{u}}=0$, and $\gamma_{\mathrm{d}}=L$, where $L$ is the horizontal length of the domain. In summary, the domains are defined as

$$
\begin{aligned}
\Omega & =\left\{\boldsymbol{x} \mid(x, y) \in \omega, z_{b}(x, y, t) \leq z \leq h(x, y, t)\right\}, \\
\Gamma_{\mathrm{s}} & =\{\boldsymbol{x} \mid(x, y) \in \omega, z=h(x, y, t)\}, \\
\Gamma_{\mathrm{b}} & =\left\{\boldsymbol{x} \mid(x, y) \in \omega, z=z_{b}(x, y, t)\right. \\
& \left.=b(x, y), x<x_{\mathrm{GL}}(y)\right\}, \\
\Gamma_{\mathrm{w}} & =\left\{\boldsymbol{x} \mid(x, y) \in \omega, z=z_{b}(x, y, t), x>x_{\mathrm{GL}}(y)\right\}, \\
\Gamma_{\mathrm{u}} & =\left\{\boldsymbol{x} \mid(x, y) \in \gamma_{\mathrm{u}}, z_{b}(x, y, t) \leq z \leq h(x, y, t)\right\}, \\
\Gamma_{\mathrm{d}} & =\left\{\boldsymbol{x} \mid(x, y) \in \gamma_{\mathrm{d}}, z_{b}(x, y, t) \leq z \leq h(x, y, t)\right\} .
\end{aligned}
$$

Before the forward FS equations for the evolution of the ice surface $\Gamma_{\mathrm{s}}$ and the ice velocity in $\Omega$ can be given, further notation needs to be introduced: ice density is denoted by $\rho$, accumulation and/or ablation rate on $\Gamma_{\mathrm{s}}$ by $a_{\mathrm{s}}$, and gravitational acceleration by $g$. The values of these physical parameters are given in Table 1 . On $\Gamma_{\mathrm{s}}, \boldsymbol{h}=\left(h_{x}, h_{y},-1\right)^{\mathrm{T}}$ describes the spatial gradient of the ice surface (in two vertical 


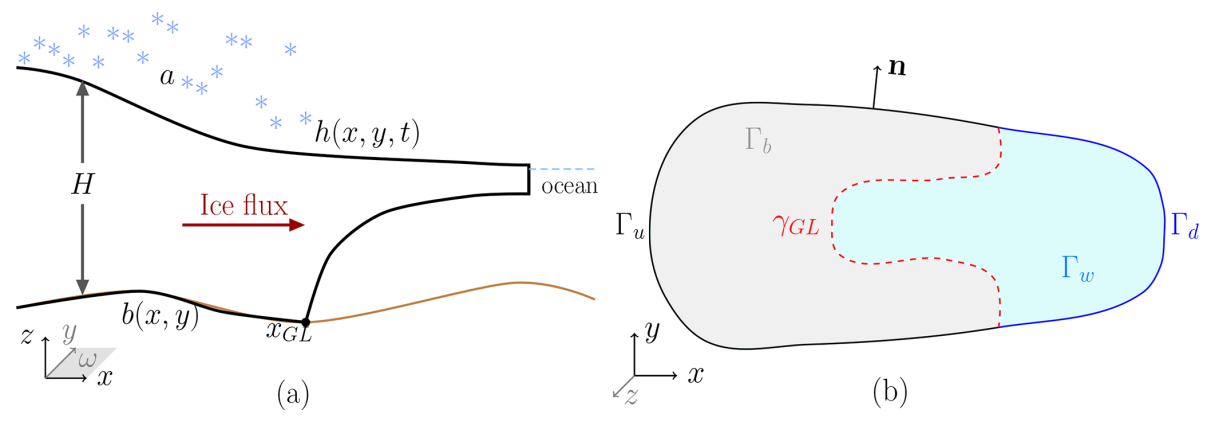

Figure 1. A schematic view of an ice sheet in the (a) $x-z$ plane and (b) $x-y$ plane.

dimensions $\left.\boldsymbol{h}=\left(h_{x},-1\right)^{\mathrm{T}}\right)$. The strain rate $\mathbf{D}$ and the viscosity $\eta$ are given by

$$
\mathbf{D}(\boldsymbol{u})=\frac{1}{2}\left(\nabla \boldsymbol{u}+\nabla \boldsymbol{u}^{\mathrm{T}}\right), \eta(\boldsymbol{u})=\frac{1}{2} A^{-\frac{1}{n}}\left(\operatorname{tr}^{2}(\boldsymbol{u})\right)^{\frac{1-n}{2 n}},
$$

where $\operatorname{tr} \mathbf{D}^{2}$ is the trace of $\mathbf{D}^{2}$. The rate factor $A$ in (2) depends on the temperature, and Glen's flow law determines $n>0$, here taken to be $n=3$. The stress tensor is

$\boldsymbol{\sigma}(\boldsymbol{u}, p)=2 \eta \mathbf{D}(\boldsymbol{u})-\mathbf{I} p$

where $p$ is the isotropic pressure and $\mathbf{I}$ is the identity matrix.

Turning to the ice base, the basal stress on $\Gamma_{b}$ is related to the basal velocity using an empirical friction law $\mathbf{T} \boldsymbol{\sigma} \boldsymbol{n}=-\beta \mathbf{T} \boldsymbol{u}$, where a projection (Petra et al., 2012) on the tangential plane of $\Gamma_{\mathrm{b}}$ is denoted by $\mathbf{T}=\mathbf{I}-\boldsymbol{n} \otimes \boldsymbol{n}$, where the Kronecker outer product between two vectors $\boldsymbol{a}$ and $\boldsymbol{c}$ or two matrices $\mathbf{A}$ and $\mathbf{C}$ is defined by

$(\boldsymbol{a} \otimes \boldsymbol{c})_{i j}=a_{i} c_{j},(\mathbf{A} \otimes \mathbf{C})_{i j k l}=A_{i j} C_{k l}$.

The friction coefficient has a general form $\beta(\boldsymbol{u}, \boldsymbol{x}, t)=$ $C(\boldsymbol{x}, t) f(\boldsymbol{u})$, where $C(\boldsymbol{x}, t)$ is independent of the velocity $\boldsymbol{u}$ and $f(\boldsymbol{u})$ represents some linear or nonlinear function of $\boldsymbol{u}$. For instance, $f(\boldsymbol{u})=\|\boldsymbol{u}\|^{m-1}$ with the norm $\|\boldsymbol{u}\|=(\boldsymbol{u} \cdot \boldsymbol{u})^{\frac{1}{2}}$ introduces a Weertman-type friction law (Weertman, 1957) on $\omega$ with a Weertman friction coefficient $C(\boldsymbol{x}, t) \geq 0$ and an exponent parameter $m>0$. Common choices of $m$ are $\frac{1}{3}$ and 1.

With these prerequisites at hand, the forward FS equations and the advection equation for the ice sheet's elevation and velocity for incompressible ice flow are

$$
\begin{aligned}
& h_{t}+\boldsymbol{h} \cdot \boldsymbol{u}=a_{\mathrm{s}}, \quad \text { on } \Gamma_{\mathrm{s}}, \quad t \geq 0, \\
& h(\boldsymbol{x}, 0)=h_{0}(\boldsymbol{x}), \boldsymbol{x} \in \omega, \quad h(\boldsymbol{x}, t)=h_{\gamma}(\boldsymbol{x}, t), \boldsymbol{x} \in \gamma_{\mathrm{u}}, \\
& -\nabla \cdot \boldsymbol{\sigma}(\boldsymbol{u}, p)=-\nabla \cdot(2 \eta(\boldsymbol{u}) \mathbf{D}(\boldsymbol{u}))+\nabla p=\rho \boldsymbol{g}, \\
& \quad \nabla \cdot \boldsymbol{u}=0, \text { in } \Omega(t), \\
& \boldsymbol{\sigma} \boldsymbol{n}=\mathbf{0}, \text { on } \Gamma_{\mathrm{s}}, \\
& \mathbf{T} \boldsymbol{\sigma} \boldsymbol{n}=-C f(\mathbf{T} \boldsymbol{u}) \mathbf{T} \boldsymbol{u}, \boldsymbol{n} \cdot \boldsymbol{u}=0, \text { on } \Gamma_{\mathrm{b}},
\end{aligned}
$$

where $h_{0}(\boldsymbol{x})>b(x, y)$ is the initial surface elevation and $h_{\gamma}(\boldsymbol{x}, t)$ is a given height on the inflow boundary. In the floating ice, the equations and the boundary conditions are as above plus an equation for the free surface $z_{b}$ with $z_{b} \geq b$ and a boundary condition on the wetted ice $\Gamma_{\mathrm{w}}$ :

$$
\begin{aligned}
& z_{b t}+u z_{b x}+v z_{b y}-w=a_{\mathrm{b}}, \text { on } \Gamma_{\mathrm{w}}, \quad t \geq 0, \\
& \boldsymbol{\sigma} \boldsymbol{n}=-p_{\mathrm{w}} \boldsymbol{n}, \text { on } \Gamma_{\mathrm{w}},
\end{aligned}
$$

where $a_{\mathrm{b}}$ is the basal mass balance and $p_{\mathrm{w}}$ is the water pressure at $\Gamma_{\mathrm{w}}$. With the sea level at $z=0$ and the water density $\rho_{\mathrm{w}}, p_{\mathrm{w}}=-\rho_{\mathrm{w}} g z_{b}$.

The solution at the grounding line satisfies a nonlinear complementarity problem. Let $d$ be the distance between the ice base and the bedrock such that

$d(\boldsymbol{x}, t)=z_{b}(\boldsymbol{x}, t)-b(\boldsymbol{x})$.

On $\Gamma_{\mathrm{w}}$, we have $d(\boldsymbol{x}, t)>0$ and $\boldsymbol{n} \cdot \boldsymbol{\sigma} \boldsymbol{n}+p_{\mathrm{w}}=0$ and on $\Gamma_{\mathrm{b}}$, we have $d(\boldsymbol{x}, t)=0$ and $\boldsymbol{n} \cdot \boldsymbol{\sigma} \boldsymbol{n}+p_{\mathrm{w}}<0$. The complementarity relation at the ice base $\Gamma_{\mathrm{b}} \cup \Gamma_{\mathrm{w}}$ can be summarized as

$d(\boldsymbol{x}, t) \geq 0, \quad \boldsymbol{n} \cdot \boldsymbol{\sigma} \boldsymbol{n}+p_{\mathrm{W}} \leq 0, \quad d(\boldsymbol{x}, t)\left(\boldsymbol{n} \cdot \boldsymbol{\sigma} \boldsymbol{n}+p_{\mathrm{w}}\right)=0$.

There are additional constraints on the solution. For example, the thickness of the ice is non-negative $H \geq 0$, there is a lower bound on the velocity in Weertmann's friction law, and there is an upper bound on the viscosity $\eta$. These conditions have to be handled in a numerical solution of the equations but are not discussed further here.

The boundary conditions for the velocity on $\Gamma_{\mathrm{u}}$ and $\Gamma_{\mathrm{d}}$ are of Dirichlet type such that

$\left.\boldsymbol{u}\right|_{\Gamma_{\mathrm{u}}}=\boldsymbol{u}_{\mathrm{u}},\left.\quad \boldsymbol{u}\right|_{\Gamma_{\mathrm{d}}}=\boldsymbol{u}_{\mathrm{d}}$,

where $\boldsymbol{u}_{\mathrm{u}}$ and $\boldsymbol{u}_{\mathrm{d}}$ are known. These are general settings of the inflow and outflow boundaries which keep the formulation of the adjoint equations as simple as in Petra et al. (2014). Should $\Gamma_{\mathrm{u}}$ be at the ice divide, the horizontal velocity is set to $\left.\boldsymbol{u}\right|_{\Gamma_{\mathrm{u}}}=\mathbf{0}$. The ice velocity at the calving front is defined as $\boldsymbol{u}_{\mathrm{d}}$ to simplify the analysis. The vertical component of $\boldsymbol{\sigma} \boldsymbol{n}$ vanishes on $\Gamma_{\mathrm{u}}$.

\subsection{Shallow-shelf approximation}

The three-dimensional FS problem (4) in $\Omega$ can be reduced to a two-dimensional, horizontal problem with $\boldsymbol{x}=(x, y) \in \omega$ 
by adopting the SSA, in which only $\boldsymbol{u}=\left(u_{1}, u_{2}\right)^{\mathrm{T}}$ is considered. This is because the basal shear stress is negligibly small at the base of the floating part of the ice mass, viz. the ice shelf, rendering the horizontal velocity components almost constant in the $z$ direction (MacAyeal, 1989). The SSA is often also used in regions of fast flow over lubricated bedrock (MacAyeal, 1989).

The simplifications associated with adopting the SSA imply that the viscosity (see 2 for the FS model) is now given by

$$
\begin{aligned}
& \eta(\boldsymbol{u})= \\
& \frac{1}{2} A^{-\frac{1}{n}}\left(u_{1 x}^{2}+u_{2 y}^{2}+\frac{1}{4}\left(u_{1 y}+u_{2 x}\right)^{2}+u_{1 x} u_{2 y}\right)^{\frac{1-n}{2 n}} \\
& =\frac{1}{2} A^{-\frac{1}{n}}\left(\frac{1}{2} \mathbf{B}: \mathbf{D}\right)^{\frac{1-n}{2 n}},
\end{aligned}
$$

where $\mathbf{B}(\boldsymbol{u})=\mathbf{D}(\boldsymbol{u})+\nabla \cdot \boldsymbol{u} \mathbf{I}$, with $\nabla \cdot \boldsymbol{u}=\operatorname{tr} \mathbf{D}(\boldsymbol{u})$. This $\eta$ differs from (2) because $\mathbf{B} \neq \mathbf{D}$ due to the cryostatic approximation of $p$ in the SSA. In (7), the Frobenius inner product between two matrices $\mathbf{A}$ and $\mathbf{C}$ is used, defined by $\mathbf{A}: \mathbf{C}=\sum_{i j} \mathbf{A}_{i j} \mathbf{C}_{i j}$. The vertically integrated stress tensor $\boldsymbol{S}(\boldsymbol{u})$ (cf. 3 for the FS model) is given by

$\boldsymbol{\varsigma}(\boldsymbol{u})=2 H \eta \mathbf{B}(\boldsymbol{u})$,

where $H$ is the ice thickness (see Fig. 1). The friction law in the SSA model is defined as in the FS case. Note that basal velocity is replaced by the horizontal velocity. This is possible because vertical variations in the horizontal velocity are neglected in SSA. Then, Weertman's law is $\beta(\boldsymbol{u}, \boldsymbol{x}, t)=C(\boldsymbol{x}, t) f(\boldsymbol{u})=C(\boldsymbol{x}, t)\|\boldsymbol{u}\|^{m-1}$ with a friction coefficient $C(\boldsymbol{x}, t) \geq 0$, just as in the FS model. In summary, the forward equations describing the evolution of the ice surface and ice velocities based on an SSA model (in which $\boldsymbol{u}$ is not divergence-free) read

$$
\begin{aligned}
& H_{t}+\nabla \cdot(\boldsymbol{u} H)=a, \quad t \geq 0, \boldsymbol{x} \in \omega, \\
& h(\boldsymbol{x}, 0)=h_{0}(\boldsymbol{x}), \boldsymbol{x} \in \omega, \quad h(\boldsymbol{x}, t)=h_{\gamma}(\boldsymbol{x}, t), \boldsymbol{x} \in \gamma_{\mathrm{u}}, \\
& \nabla \cdot \boldsymbol{\varsigma}-C f(\boldsymbol{u}) \boldsymbol{u}=\rho g H \nabla h, \boldsymbol{x} \in \omega, \\
& \boldsymbol{n} \cdot \boldsymbol{u}(\boldsymbol{x}, t)=u_{\mathrm{u}}(\boldsymbol{x}, t), \boldsymbol{x} \in \gamma_{\mathrm{u}}, \boldsymbol{n} \cdot \boldsymbol{u}(\boldsymbol{x}, t)=u_{\mathrm{d}}(\boldsymbol{x}, t), \\
& \quad \boldsymbol{x} \in \gamma_{\mathrm{d}}, \\
& \boldsymbol{t} \cdot \boldsymbol{\varsigma} \boldsymbol{n}=-C_{\gamma} f_{\gamma}(\boldsymbol{t} \cdot \boldsymbol{u}) \boldsymbol{t} \cdot \boldsymbol{u}, \boldsymbol{x} \in \gamma_{g}, \\
& \boldsymbol{t} \cdot \boldsymbol{\varsigma} \boldsymbol{n}=0, \boldsymbol{x} \in \gamma_{\mathrm{w}} .
\end{aligned}
$$

Above, $t$ is the tangential vector on $\gamma=\gamma_{\mathrm{u}} \cup \gamma_{\mathrm{d}}$ such that $\boldsymbol{n} \cdot \boldsymbol{t}=0$ and $a=a_{\mathrm{s}}-a_{\mathrm{b}}$. The inflow and outflow normal velocities $u_{\mathrm{u}} \leq 0$ and $u_{\mathrm{d}}>0$ are specified on $\gamma_{\mathrm{u}}$ and $\gamma_{\mathrm{d}}$. The lateral side of the ice $\gamma$ is split into $\gamma_{g}$ and $\gamma_{\mathrm{w}}$ with $\gamma=\gamma_{g} \cup \gamma_{\mathrm{w}}$. There is friction in the tangential direction on $\gamma_{g}$ which depends on the tangential velocity $\boldsymbol{t} \cdot \boldsymbol{u}$ with the friction coefficient $C_{\gamma}$ and friction function $f_{\gamma}$. There is no friction on the wet boundary $\gamma_{\mathrm{w}}$. When the equations are solved for the grounded part, $H_{t}=h_{t}$ with a time-independent topography.
A flotation criterion determines the position of the grounding line (see, e.g., Seroussi et al. (2014)). The thickness $H$ is compared to a flotation height $H_{\mathrm{f}}$ given by

$H_{\mathrm{f}}=-\rho_{\mathrm{w}} z_{b} / \rho$.

If $H>H_{\mathrm{f}}$ then the ice is grounded and $C>0$ in (9). If $H<H_{\mathrm{f}}$ then it is floating with $C=0$. The grounding line is defined by $H=H_{\mathrm{f}}$.

For ice sheets that develop an ice shelf, the latter is assumed to be at hydrostatic equilibrium. In such a case, a calving front boundary condition (Schoof, 2007; van der Veen, 1996) is applied at $\gamma_{\mathrm{d}}$, in the form of the depth-integrated stress balance ( $\rho_{\mathrm{w}}$ is the density of seawater):

$\boldsymbol{\zeta}(\boldsymbol{u}) \cdot \boldsymbol{n}=\frac{1}{2} \rho g H^{2}\left(1-\frac{\rho}{\rho_{\mathrm{w}}}\right) \boldsymbol{n}, \boldsymbol{x} \in \gamma_{\mathrm{d}}$.

\subsubsection{The flow line model of SSA}

In this section, the SSA equations are presented for the case of an idealized, two-dimensional vertical sheet in the $x$ $z$ plane (see Fig. 1). The forward SSA equations are derived from (9) by letting $H$ and $u_{1}$ be independent of $y$ and setting $u_{2}=0$. Since there is no lateral force, $C_{\gamma}=0$. The position of the grounding line is denoted by $x_{\mathrm{GL}}$, and $\Gamma_{\mathrm{b}}=\left[0, x_{\mathrm{GL}}\right], \Gamma_{\mathrm{w}}=\left(x_{\mathrm{GL}}, L\right]$. Basal friction $C$ is positive and constant where the ice sheet is grounded on bedrock, while $C=0$ at the floating ice shelf's lower boundary. To simplify notation, we let $u=u_{1}$. The forward equations thus become

$H_{t}+(u H)_{x}=a, 0 \leq t \leq T, 0 \leq x \leq L$,

$h(x, 0)=h_{0}(x), h(0, t)=h_{L}(t)$,

$\left(H \eta u_{x}\right)_{x}-C f(u) u-\rho g H h_{x}=0$,

$u(0, t)=u_{\mathrm{u}}(t), u(L, t)=u_{\mathrm{d}}(t)$,

where $u_{\mathrm{u}}$ is the speed of the ice flux at $x=0$ and $u_{\mathrm{d}}$ is the speed at the calving front at $x=L$. If $x=0$ is at the ice divide, then $u_{\mathrm{u}}=0$. By the stress balance (10), the calving front satisfies

$u_{x}(L, t)=A\left[\frac{\rho g H(L, t)}{4}\left(1-\frac{\rho}{\rho_{\mathrm{w}}}\right)\right]^{n}$.

Assuming that $u>0$ and $u_{x}>0$, the viscosity becomes $\eta=$ $2 A^{-\frac{1}{n}} u_{x}^{\frac{1-n}{n}}$, and the friction term with a Weertman law turns into $C f(u) u=C u^{m}$.

\subsubsection{The two-dimensional forward steady-state solution}

We now discuss the steady-state solutions to the system (11). Except for letting all time derivatives vanish, even the longitudinal stress can be ignored in the steady-state solution (see 
Table 1. The model parameters.

\begin{tabular}{|c|c|}
\hline Parameter & Quantity \\
\hline$a=0.3 \mathrm{~m} \mathrm{yr}^{-1}$ & Surface mass balance \\
\hline$A=1.38 \times 10^{-24} \mathrm{~s}^{-1} \mathrm{~Pa}^{-3}$ & Rate factor of Glen's flow law \\
\hline$C=7.624 \times 10^{6} \mathrm{Pam}^{-1 / 3} \mathrm{~s}^{1 / 3}$ & Basal friction coefficient \\
\hline$g=9.8 \mathrm{~m} \mathrm{~s}^{-2}$ & Acceleration of gravity \\
\hline$m=1 / 3$ & Friction law exponent \\
\hline$n=3$ & Flow-law exponent \\
\hline$\rho=900 \mathrm{~kg} \mathrm{~m}^{-3}$ & Ice density \\
\hline
\end{tabular}

Schoof (2007)). With a sliding law in the form $f(u)=u^{m-1}$ and the thickness given at $x_{\mathrm{GL}},(11)$ thus reduces to

$$
\begin{aligned}
& (u H)_{x}=a, 0 \leq x \leq x_{\mathrm{GL}}, \\
& H\left(x_{\mathrm{GL}}\right)=H_{\mathrm{GL}}, \\
& -C u^{m}-\rho g H h_{x}=0, \\
& u(0)=0 .
\end{aligned}
$$

The solution to the forward equation (12) is derived for the case when $a$ and $C$ are constant (for details, see D3 and D4 in Appendix D):

$$
\begin{aligned}
& H(x)=\left(H_{\mathrm{GL}}^{m+2}+\frac{m+2}{m+1} \frac{C a^{m}}{\rho g}\left(x_{\mathrm{GL}}^{m+1}-x^{m+1}\right)\right)^{\frac{1}{m+2}}, \\
& 0 \leq x \leq x_{\mathrm{GL}} \\
& H(x)=H_{\mathrm{GL}}, x_{\mathrm{GL}}<x<L, \\
& u(x)=\frac{a x}{H}, 0 \leq x \leq x_{\mathrm{GL}}, u(x)=\frac{a x}{H_{\mathrm{GL}}}, \\
& x_{\mathrm{GL}}<x<L .
\end{aligned}
$$

The solution is normalized with the ice thickness $H_{\mathrm{GL}}=$ $H\left(x_{\mathrm{GL}}\right)$ at the grounding line. Both $x_{\mathrm{GL}}$ and $H_{\mathrm{GL}}$ are assumed to be known in the formula. Similar equations to those in (12) are derived in Nye (1959) using the properties of large ice sheets. A formula for $H(x)$ resembling (13) and involving $H(0)$ is the solution of the equations. By including the longitudinal stress in the ice, an approximate, more complicated expression for $H(x)$ is obtained in Weertman (1961).

Figure 2 displays solutions from (13) obtained with data from the Marine Ice Sheet Model Intercomparison Project (MISMIP) (Pattyn et al., 2012) test case EXP 1 chosen in Cheng and Lötstedt (2020). The modeling parameters in (13) are given in Table 1 . The ice sheet flows from $x=0$ to $L=$ $1.6 \times 10^{6} \mathrm{~m}$ on a single slope bed defined by $b(x)=720-$ $\frac{778.5 x}{7.5 \times 10^{5}}$ and lifts from it at the grounding line position $x_{\mathrm{GL}}=$ $1.035 \times 10^{6} \mathrm{~m}$. As $x$ approaches $x_{\mathrm{GL}}, H$ decreases to become $H_{\mathrm{GL}}$ at $x_{\mathrm{GL}}$ in Fig. $2 \mathrm{~b}$.

The larger the friction coefficient $C$ and accumulation rate $a$ are, the steeper the decrease in $H$ is in (13). The numerator in $u$ increases and the denominator decreases when $x \rightarrow x_{\mathrm{GL}}$, resulting in a rapid increase in $u$. The MISMIP example is such that the SSA solution is close to the FS solution. Numerical experiments in Cheng and Lötstedt (2020) show that an accurate solution compared to the FS and SSA solutions is obtained with $u$ and $H$ in (13) solving (12).

Finally, it is noted that an alternative solution to (11) valid for the floating ice shelf, $x>x_{\mathrm{GL}}$, but under the restraining assumption of $H(x)$ being linear in $x$, is found in Greve and Blatter (2009).

\section{Adjoint equations}

In this section, the adjoint equations are discussed, as emerging in a FS framework (Sect. 3.1) and in a SSA framework (Sect. 3.2), respectively. We derive the adjoint equations for the grounded part of the ice sheet. There the friction coefficient can be perturbed with $\delta C \neq 0$, and a perturbation $\delta b$ of the topography has a direct influence on the flow of ice. The adjoint equations follow from the Lagrangian based on the forward equations after partial integration. Lengthy derivations have been moved to Appendix A. A numerical example based on the MISMIP (Pattyn et al., 2012) used also in Cheng and Lötstedt (2020) illustrates the findings presented.

On the ice surface $\Gamma_{\mathrm{s}}$ and over the time interval $[0, T]$, we consider the functional $\mathcal{F}$ :

$$
\mathcal{F}=\int_{0}^{T} \int_{\Gamma_{\mathrm{S}}} F(\boldsymbol{u}, h) \mathrm{d} \boldsymbol{x} \mathrm{d} t .
$$

We wish to determine its sensitivity to perturbations in both the friction coefficient $C(x, t)$ at the base of the ice and the topography of the base itself $b(\boldsymbol{x})$, which is a smooth function in $\boldsymbol{x}$. We distinguish two cases: $\boldsymbol{u}$ and $h$ satisfy either the FS equation (4) or the SSA equation (9). Given $\mathcal{F}$, the forward solution $(\boldsymbol{u}, p, h)$ to (4) or $(\boldsymbol{u}, h)$ to (9), and the adjoint solution $(\boldsymbol{v}, q, \psi)$ or $(\boldsymbol{v}, \psi)$ to the adjoint FS and adjoint SSA equations (both derived in the following and in Appendix A), we introduce a Lagrangian $\mathcal{L}(\boldsymbol{u}, p, h ; \boldsymbol{v}, q, \psi ; b, C)$. The Lagrangian for the FS equations is

$$
\begin{aligned}
& \mathcal{L}(\boldsymbol{u}, p, h ; \boldsymbol{v}, q, \psi ; C)= \\
& \quad \int_{0}^{T} \int_{\Gamma_{\mathrm{S}}} F(\boldsymbol{u}, h)+\psi\left(h_{t}+\boldsymbol{h} \cdot \boldsymbol{u}-a\right) \mathrm{d} \boldsymbol{x} \mathrm{d} t \\
& +\int_{0}^{T} \int_{\omega} \int_{b}^{h}-\boldsymbol{v} \cdot(\nabla \cdot \boldsymbol{\sigma}(\boldsymbol{u}, p))-\rho \boldsymbol{g} \cdot \boldsymbol{v} \\
& -q \nabla \cdot \boldsymbol{u} \mathrm{d} \boldsymbol{x} \mathrm{d} t,
\end{aligned}
$$

with the Lagrange multipliers $v, q$, and $\psi$ corresponding to the forward equations for $\boldsymbol{u}, p$, and $h$. The effect of perturbations $\delta C$ and $\delta b$ in $C$ and $b$ on $\mathcal{F}$ is given by the perturbation $\delta \mathcal{L}$, viz.

$$
\begin{gathered}
\delta \mathcal{F}=\delta \mathcal{L}=\mathcal{L}(\boldsymbol{u}+\delta \boldsymbol{u}, p+\delta p, h+\delta h ; \boldsymbol{v}+\delta \boldsymbol{v}, q+\delta q, \\
\psi+\delta \psi ; b+\delta b, C+\delta C)-\mathcal{L}(\boldsymbol{u}, p, h ; \boldsymbol{v}, q, \psi ; b, C) .
\end{gathered}
$$




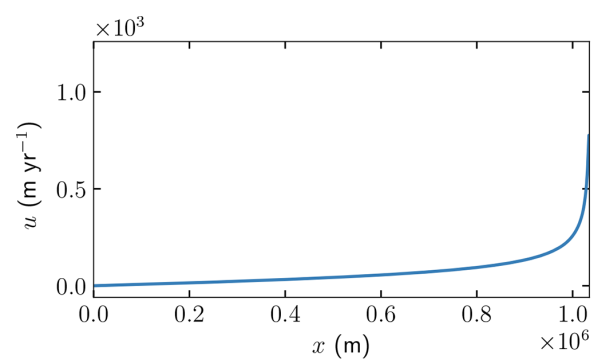

(a)

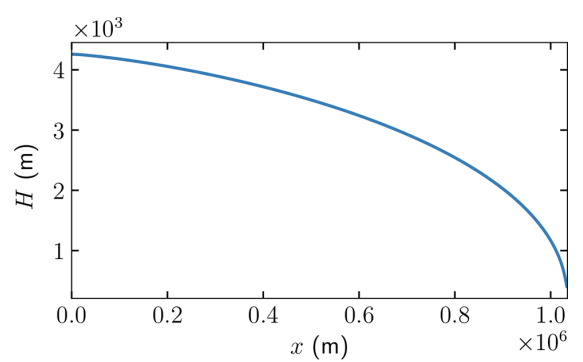

(b)

Figure 2. The analytical solutions $u(x)$ and $H(x)$ in (13) for a grounded ice in [0, $\left.x_{\mathrm{GL}}\right]$.

Examples of $F(\boldsymbol{u}, h)$ in (14) are $F=\left\|\boldsymbol{u}-\boldsymbol{u}_{\mathrm{obs}}\right\|^{2}$, and $F=\left|h-h_{\text {obs }}\right|^{2}$ in an inverse problem, in which the task is to find $b$ and $C$ such that they match observations $\boldsymbol{u}_{\mathrm{obs}}$ and $h_{\mathrm{obs}}$ at the surface $\Gamma_{\mathrm{s}}$ (see also Gillet-Chaulet et al., 2016; Isaac et al., 2015; Morlighem et al., 2013; and Petra et al., 2012). Another example is $F(\boldsymbol{u}, h)=\frac{1}{T} u_{1}(\boldsymbol{x}, t) \delta\left(\boldsymbol{x}-\boldsymbol{x}_{*}\right)$ with the Dirac delta $\delta$ at $\boldsymbol{x}_{*}$ to measure the time-averaged horizontal velocity $u_{1}$ at $\boldsymbol{x}_{*}$ on the ice surface $\Gamma_{\mathrm{s}}$ with

$\mathcal{F}=\int_{0}^{T} \int_{\Gamma_{\mathrm{S}}} F(\boldsymbol{u}, h) \mathrm{d} \boldsymbol{x} \mathrm{d} t=\frac{1}{T} \int_{0}^{T} u_{1}\left(\boldsymbol{x}_{*}, t\right) \mathrm{d} t$,

where $T$ is the duration of the observation at $\Gamma_{\mathrm{s}}$. If the horizontal velocity is observed at $\left(x_{*}, t_{*}\right)$ then $F(\boldsymbol{u}, h)=$ $u_{1}(\boldsymbol{x}, t) \delta\left(\boldsymbol{x}-\boldsymbol{x}_{*}\right) \delta\left(t-t_{*}\right)$ and

$\mathcal{F}=\int_{0}^{T} \int_{\Gamma_{\mathrm{S}}} F(\boldsymbol{u}, h) \mathrm{d} \boldsymbol{x} \mathrm{d} t=u_{1}\left(\boldsymbol{x}_{*}, t_{*}\right)$.

The sensitivity in $\mathcal{F}$ and $u_{1}$ in (17) or (18) to perturbations in $C$ and $b$ is then given by (16) with the forward and adjoint solutions.

The same forward and adjoint equations are solved both for the inverse problem and the sensitivity problem but with different forcing function $F$ in (14). If we are interested in how $u_{1}$ changes at the surface when $b$ and $C$ are changed at the base by given $\delta b$ and $\delta C$, then $F$ is as in (18). The forward and adjoint equations are then solved once. In the inverse problem with velocity observations, $\mathcal{F}$ is the objective function in a minimization procedure and $F=\left\|\boldsymbol{u}-\boldsymbol{u}_{\mathrm{obs}}\right\|^{2}$. The change $\delta \mathcal{F}$ in $\mathcal{F}$ is of interest when $C$ and $b$ are changed during the solution procedure. In order to minimize $\mathcal{F}, \delta C$ and $\delta b$ are chosen such that $\delta \mathcal{F}<0$ and $\mathcal{F}$ decreases with $C+\delta C$ and $b+\delta b$ and $\boldsymbol{u}$ is closer to $\boldsymbol{u}_{\text {obs. }}$. Precisely how $\delta C$ and $\delta b$ are chosen depends on the optimization algorithm. This procedure is repeated iteratively, and $b$ and $C$ are updated by $b+\delta b$ and $C+\delta C$ until $\delta \mathcal{F} \rightarrow 0$ and $\mathcal{F}$ has reached a minimum. The forward and adjoint equations have to be solved in each iteration in the inverse problem.

\subsection{Adjoint equations based on the FS model}

In this section, we introduce the adjoint equations and the perturbation of the Lagrangian function. The detailed derivations of (19) and (22) below are given in Appendix A, starting from the weak form of the FS equation (4) on $\Omega$ and by using integration by parts, and applying boundary conditions as in Martin and Monnier (2014) and Petra et al. (2012).

The definition of the Lagrangian $\mathcal{L}$ for the FS equations is given in (15) and (A15) in Appendix A. To determine $(\boldsymbol{v}, q, \psi)$ in $(15)$, the following adjoint problem is solved:

$$
\begin{aligned}
& \psi_{t}+\nabla \cdot(\boldsymbol{u} \psi)-\boldsymbol{h} \cdot \boldsymbol{u}_{z} \psi=F_{h}+F_{\boldsymbol{u}} \cdot \boldsymbol{u}_{z}, \text { on } \Gamma_{\mathrm{s}}, \\
& \quad 0 \leq t \leq T, \\
& \psi(\boldsymbol{x}, T)=0, \psi(\boldsymbol{x}, t)=0, \text { on } \Gamma_{\mathrm{d}}, \\
& -\nabla \cdot \tilde{\boldsymbol{\sigma}}(\boldsymbol{v}, q)=-\nabla \cdot(2 \tilde{\boldsymbol{\eta}}(\boldsymbol{u}) \star \mathbf{D}(\boldsymbol{v}))+\nabla q=\mathbf{0}, \\
& \quad \nabla \cdot \boldsymbol{v}=0, \text { in } \Omega(t), \\
& \tilde{\boldsymbol{\sigma}}(\boldsymbol{v}, q) \boldsymbol{n}=-\left(F_{\boldsymbol{u}}+\psi \boldsymbol{h}\right), \text { on } \Gamma_{\mathrm{s}}, \\
& \mathbf{T} \tilde{\boldsymbol{\sigma}}(\boldsymbol{v}, q) \boldsymbol{n}=-C f(\mathbf{T} \boldsymbol{u})\left(\mathbf{I}+\mathbf{F}_{b}(\mathbf{T} \boldsymbol{u})\right) \mathbf{T} \boldsymbol{v}, \text { on } \Gamma_{\mathrm{b}}, \\
& \boldsymbol{n} \cdot \boldsymbol{v}=0, \text { on } \Gamma_{\mathrm{b}},
\end{aligned}
$$

where the derivatives of $F$ with respect to $\boldsymbol{u}$ and $h$ are

$$
F_{\boldsymbol{u}}=\left(\frac{\partial F}{\partial u_{1}}, \frac{\partial F}{\partial u_{2}}, \frac{\partial F}{\partial u_{3}}\right)^{\mathrm{T}}, \quad F_{h}=\frac{\partial F}{\partial h} .
$$

Note that (19) consists of equations for the adjoint elevation $\psi$, the adjoint velocity $\boldsymbol{v}$, and the adjoint pressure $q$. The equations are the same as when the derivatives are computed in the inverse problem except for the terms depending on $F$, which is the misfit between the numerical solution and the observation in the inverse problem. Compared to the steadystate adjoint equation for the FS equations discussed in Petra et al. (2012), an advection equation is added in (19) for the Lagrange multiplier $\psi(\boldsymbol{x}, t)$ on $\Gamma_{\mathrm{s}}$ with a right-hand side depending on the observation function $F$ and one term depending on $\psi$ in the boundary condition on $\Gamma_{\mathrm{s}}$. The adjoint elevation equation for $\psi$ can be solved independently of the adjoint stress equation since it is independent of $\boldsymbol{v}$. If $h$ is observed and $F_{h} \neq 0$ and $F_{\boldsymbol{u}}=\mathbf{0}$, then the adjoint elevation equation must be solved together with the adjoint stress equation. Otherwise, the term $\psi \boldsymbol{h}$ is ignored in the right-hand side 
of the boundary condition of the adjoint stress equation and the solution is $\boldsymbol{v}=\mathbf{0}$ with $\delta \mathcal{F}=0$ in (22); see below.

The adjoint viscosity and adjoint stress are

$$
\begin{aligned}
\tilde{\boldsymbol{\eta}}(\boldsymbol{u}) & =\eta(\boldsymbol{u})\left(\mathcal{I}+\frac{1-n}{n \mathbf{D}(\boldsymbol{u}): \mathbf{D}(\boldsymbol{u})} \mathbf{D}(\boldsymbol{u}) \otimes \mathbf{D}(\boldsymbol{u})\right), \\
\tilde{\boldsymbol{\sigma}}(\boldsymbol{v}, q) & =2 \tilde{\boldsymbol{\eta}}(\boldsymbol{u}) \star \mathbf{D}(\boldsymbol{v})-q \mathbf{I} ;
\end{aligned}
$$

cf. also Petra et al. (2012). For the rank four-tensor $\mathcal{I}$, $\mathcal{I}_{i j k l}=1$ only when $i=j=k=l$; otherwise $\mathcal{I}_{i j k l}=0$. The $\star$ operation in (20) between a rank four-tensor $\mathcal{A}$ and a rank two-tensor (viz. a matrix) $\mathbf{C}$ is defined by $(\mathcal{A} \star \mathbf{C})_{i j}=$ $\sum_{k l} \mathcal{A}_{i j k l} C_{k l}$. In general, $\mathbf{F}_{b}(\mathbf{T} \boldsymbol{u})$ in (19) is a linearization of the friction law $f(\mathbf{T} \boldsymbol{u})$ in (4) with respect to the variable $\mathbf{T} \boldsymbol{u}$. For instance, with a Weertman-type friction law, $f(\mathbf{T} \boldsymbol{u})=\|\mathbf{T} \boldsymbol{u}\|^{m-1}$,

$\mathbf{F}_{b}(\mathbf{T} \boldsymbol{u})=\frac{m-1}{\mathbf{T} \boldsymbol{u} \cdot \mathbf{T} \boldsymbol{u}}(\mathbf{T} \boldsymbol{u}) \otimes(\mathbf{T} \boldsymbol{u})$.

The perturbation of the Lagrangian function with respect to a perturbation $\delta C$ in the slip coefficient $C(\boldsymbol{x}, t)$ involves the tangential components of the forward and adjoint velocities, $\mathbf{T} \boldsymbol{u}$ and $\mathbf{T} \boldsymbol{v}$ at the ice base $\Gamma_{\mathrm{b}}$, and is given by

$\delta \mathcal{F}=\delta \mathcal{L}=\int_{0}^{T} \int_{\Gamma_{\mathrm{b}}} f(\mathbf{T} \boldsymbol{u}) \mathbf{T} \boldsymbol{u} \cdot \mathbf{T} \boldsymbol{v} \delta C \mathrm{~d} \boldsymbol{x} \mathrm{d} t$.

For this formula to be accurate, $\delta C$ has to be small. Otherwise, nonlinear effects may be of importance.

\subsubsection{Time-dependent perturbations}

Let us now investigate the effect of time-dependent perturbations in the friction parameter on modeled ice velocities and ice surface elevation. Suppose that the velocity component $u_{1 *}=u_{1}\left(\boldsymbol{x}_{*}, t_{*}\right)$ is observed at $\left(\boldsymbol{x}_{*}, t_{*}\right)$ at the ice surface as in (18) and that $t_{*}<T$, then

$u_{1 *}=\mathcal{F}=\int_{0}^{T} \int_{\Gamma_{\mathrm{s}}} F(\boldsymbol{u}) \mathrm{d} \boldsymbol{x} \mathrm{d} t$,

with $\quad F(\boldsymbol{u})=u_{1} \delta\left(\boldsymbol{x}-\boldsymbol{x}_{*}\right) \delta\left(t-t_{*}\right), F_{u_{1}}=\delta\left(\boldsymbol{x}-\boldsymbol{x}_{*}\right) \delta(t-$ $\left.t_{*}\right), F_{u_{2}}=F_{u_{3}}=0$, and $F_{h}=0$. Above, we have introduced the simplifying notation that a variable with subscript $*$ is a shorthand for it being evaluated at $\left(\boldsymbol{x}_{*}, t_{*}\right)$ or, if it is time independent, at $\boldsymbol{x}_{*}$. Here we have chosen to consider the perturbation at a certain point in space and time $\left(x_{*}, t_{*}\right)$, which is sufficient because other types of sensitivity over a certain period of time and space as in (17) are the linear combination of point-wise sensitivities.

The procedure to determine the sensitivity is as follows. First, the forward equation (4) is solved for $\boldsymbol{u}(\boldsymbol{x}, t)$ and $h(\boldsymbol{x}, t)$ from $t=0$ to $t=T$. Then, the adjoint equation (19) is solved backward in time (from $t=T$ to $t=0$ ) with $\psi(\boldsymbol{x}, T)=0$ as the corresponding final condition. Obviously, the solution for $t_{*}<t \leq T$ is $\psi(\boldsymbol{x}, t)=0$ and $\boldsymbol{v}(\boldsymbol{x}, t)=\mathbf{0}$. Letting $\boldsymbol{e}^{i}$ denote the unit vector with 1 in the $i$ th component, the boundary condition in (19) becomes $\tilde{\boldsymbol{\sigma}}(\boldsymbol{v}, q) \boldsymbol{n}=$ $-\boldsymbol{e}^{1} \delta\left(\boldsymbol{x}-\boldsymbol{x}_{*}\right) \delta\left(t-t_{*}\right)-\psi \boldsymbol{h}$ at $t=t_{*}$. For $t<t_{*}, \tilde{\boldsymbol{\sigma}}(\boldsymbol{v}, q) \boldsymbol{n}=$ $-\psi \boldsymbol{h}$. Since $\psi$ is small for $t<t_{*}$ (see Sect. 3.1.4), the dominant part of the solution is $\boldsymbol{v}(\boldsymbol{x}, t)=\boldsymbol{v}_{0}(\boldsymbol{x}) \delta\left(t-t_{*}\right)$ for some $\boldsymbol{v}_{0}$.

We start by investigating the response of ice velocities to perturbations in friction at the base: when the slip coefficient at the ice base is changed by $\delta C$, then the change in $u_{1 *}$ at $\Gamma_{\mathrm{s}}$ is, according to (22), given by

$$
\begin{aligned}
\delta u_{1 *} & =\delta \mathcal{L}=\int_{0}^{T} \int_{\Gamma_{\mathrm{b}}} f(\mathbf{T} \boldsymbol{u}) \mathbf{T} \boldsymbol{u} \cdot \mathbf{T} \boldsymbol{v} \delta C \mathrm{~d} \boldsymbol{x} \mathrm{d} t \\
& \approx \int_{\Gamma_{\mathrm{b}}} f(\mathbf{T} \boldsymbol{u}) \mathbf{T} \boldsymbol{u} \cdot \mathbf{T} \boldsymbol{v}_{0} \delta C\left(\boldsymbol{x}, t_{*}\right) \mathrm{d} \boldsymbol{x} .
\end{aligned}
$$

This implies that the perturbation $\delta u_{1 *}$ mainly depends on $\delta C$ at time $t_{*}$ and that contributions from previous $\delta C(\boldsymbol{x}, t), t<$ $t_{*}$, are small. If we observe the horizontal velocity, then it responds instantaneously in time to the change in basal friction.

Further, to investigate the response of the ice surface elevation, $h_{*}$ at $\Gamma_{\mathrm{s}}$, to perturbations in basal friction, one considers

$F(h)=h(x, t) \delta\left(\boldsymbol{x}-\boldsymbol{x}_{*}\right) \delta\left(t-t_{*}\right), F_{h}=\delta\left(\boldsymbol{x}-\boldsymbol{x}_{*}\right) \delta\left(t-t_{*}\right)$, $F_{\boldsymbol{u}}=\mathbf{0}$.

The solution of the adjoint equation (19) with $\tilde{\boldsymbol{\sigma}}(\boldsymbol{v}, q) \boldsymbol{n}=$ $-\psi \boldsymbol{h}$ at $\Gamma_{\mathrm{s}}$ for $\boldsymbol{v}(\boldsymbol{x}, t)$ is non-zero since $\psi(\boldsymbol{x}, t) \neq 0$ for $t<$ $t_{*}$.

In applied scenarios, friction at the base of an ice sheet is expected to exhibit seasonal variations. These can be expressed by $\delta C(\boldsymbol{x}, t)=\delta C_{0}(\boldsymbol{x}) \cos (2 \pi t / \tau)$, viz. a timedependent perturbation added to a stationary time average $C(\boldsymbol{x})$, with $0<\delta C_{0} \leq C$. If, for illustrational purposes, $\tau=$ 1 (1 year, from January to December), then Northern Hemisphere cold and warm seasons can in a simplified manner be associated with $n \tau, n=0,1,2, \ldots$ (winter) and $(n+1 / 2) \tau$, $n=0,1,2, \ldots$ (summer).

Assume further that $f(\mathbf{T} \boldsymbol{u}) \mathbf{T} \boldsymbol{u} \cdot \mathbf{T} \boldsymbol{v}$ is approximately constant in time. This is the case if $\boldsymbol{u}$ varies slowly in time. Then $\psi \approx$ constant and $v \approx$ constant for $t<t_{*}$. The change in ice surface elevation, $\delta h$, due to time-dependent variations in basal friction varies as

$$
\begin{aligned}
\delta h_{*} & =\delta \mathcal{L}=\int_{0}^{T} \int_{\Gamma_{\mathrm{b}}} f(\mathbf{T} \boldsymbol{u}) \mathbf{T} \boldsymbol{u} \cdot \mathbf{T} \boldsymbol{v} \delta C(\boldsymbol{x}, t) \mathrm{d} \boldsymbol{x} \mathrm{d} t \\
& \approx \mathcal{J} \int_{0}^{t_{*}} \cos (2 \pi t / \tau) \mathrm{d} t=\mathcal{J} \frac{\tau}{2 \pi} \sin \left(2 \pi t_{*} / \tau\right),
\end{aligned}
$$

where the integral $\mathcal{J}$ is

$$
\mathcal{J}=\int_{\Gamma_{\mathrm{b}}} f(\mathbf{T} \boldsymbol{u}) \mathbf{T} \boldsymbol{u} \cdot \mathbf{T} \boldsymbol{v} \delta C_{0} \mathrm{~d} \boldsymbol{x} .
$$


Obviously, from the properties of the cosine function, the friction perturbation $\delta C$ is large at $t_{*}=0, \tau / 2, \tau, \ldots$, and vanishes at $t_{*}=\tau / 4,3 \tau / 4, \ldots$. Yet, (25) shows that $\delta h_{*}=0$ at $t_{*}=0, \tau, \ldots$ (during maximal friction in the winter) and at $t_{*}=\tau / 2,3 \tau / 2, \ldots$ (during minimal friction in the summer), while $\delta h_{*} \neq 0$ when $\delta C=0$ at $t_{*}=\tau / 4,3 \tau / 4, \ldots$ in the spring and the fall. The response in $h$ by changing $C$ is delayed in phase by $\pi / 2$ or in time by $\tau / 4=0.25$ years. This is in contrast to the observation of $u_{1}$ in (24) where a perturbation in $C$ is directly visible.

Particularly in an inverse problem where the phase shift between $\delta C$ and $\delta h$ in (25) is not accounted for, if $h_{*}$ is measured in the summer with $\delta h\left(\boldsymbol{x}, t_{*}\right)=0$, then the wrong conclusion would be drawn such that there is no change in $C$.

In another example, suppose that there is an interval with a step change of $C$ with $\delta C(\boldsymbol{x}, t)=\delta C_{0}(\boldsymbol{x}) s(t)$, where $s(t)=$ 1 in the time interval $\left[t_{0}, t_{1}\right]$ and 0 otherwise. Then with $\mathcal{J}$ in (26), $\delta h_{*}$ in (25) is

$\delta h_{*} \approx \mathcal{J} \int_{0}^{t_{*}} s(t) \mathrm{d} t= \begin{cases}0, & t_{*} \leq t_{0}, \\ \mathcal{J}\left(t_{*}-t_{0}\right), & t_{0}<t_{*}<t_{1}, \\ \mathcal{J}\left(t_{1}-t_{0}\right), & t_{*}>t_{1} .\end{cases}$

The effect of the basal perturbation successively increases in the elevation when $t_{*}>t_{0}$ and stays at a higher level for $t_{*}>t_{1}$ when $\delta C$ has vanished.

\subsubsection{Example with seasonal variation}

To illustrate the phase delay in an oscillatory perturbation, a two-dimensional numerical example is shown in Fig. 3, where the timescale and friction coefficient are chosen as follows: $\tau=1$ year, $\delta C(x, t)=0.01 C \cos (2 \pi t)$ in $x \in$ $[0.9,1.0] \times 10^{6} \mathrm{~m}$. We reuse the MISMIP (Pattyn et al., 2012) test case EXP 1 as in Sect. 2.2.2. The parameters of the setup are the same as in Fig. 2 and are given in Table 1. The variables $u_{1}$ and $h$ are observed at $x \in[0.85,1.02] \times 10^{6} \mathrm{~m}$. The steady-state solution of the forward equation with the GL located at $x_{\mathrm{GL}}=1.035 \times 10^{6} \mathrm{~m}$ is perturbed by $\delta \boldsymbol{u}_{1}$ and $\delta h$ when $C$ is perturbed by $\delta C$ as expressed in formulas $\delta u_{1}=u_{1}(C+\delta C)-u_{1}(C)$ and $\delta h=h(C+\delta C)-h(C)$. After perturbation, the GL position will oscillate in time. The ice sheet is simulated by FS with Elmer/Ice (Gagliardini et al., 2013) for 10 years using the method implemented there for the position of the grounding line.

Figure 3 shows that the perturbations $\delta u_{1}$ and $\delta h$ in the grounded part of the ice sheet, specifically at $x_{*}=$ $0.85,0.9,0.95,1.0$, and $1.02 \times 10^{6} \mathrm{~m}$ for which individual panels are shown, oscillate regularly with a period of 1 year. The perturbations are small outside the interval $[0.9,1.0] \times$ $10^{6}$. The initial condition at $t=0$ is the steady-state solution of the MISMIP problem and the FS solution with a variable $C$ is essentially that steady-state solution plus a small oscillatory perturbation, as in Fig. 3.

The weight $f(\mathbf{T} \boldsymbol{u}) \mathbf{T} \boldsymbol{u} \cdot \mathbf{T} \boldsymbol{v}_{0}$ in (24) is negative, and an increase in the friction, $\delta C>0$, leads to a decrease in the veloc- ity, and $\delta C<0$ increases the velocity in all panels of Fig. 3 . The velocities $\delta u_{1}$ and the surface elevations $\delta h$ are separated by a phase shift in time, $\Delta \phi=\pi / 2$, as predicted by (24) and (25).

The weight in (25) for $\delta C_{0}$ in the integral over $\boldsymbol{x}$ changes sign when the observation point is passing from $x_{*}=0.9 \times$ $10^{6}$ to $1.0 \times 10^{6}$, explaining why the shift changes sign in the red dashed lines shown in the two lower panels of Fig. 3.

\subsubsection{The sensitivity problem and the inverse problem}

From a theoretical point of view, it is interesting to note that there is a relation between the sensitivity problem where the effect of perturbed parameters in the forward model is estimated and the inverse problem used to infer "unobservable" parameters such as basal friction from observable data, e.g., ice velocity at the ice sheet surface. The same adjoint equation (19) are solved in both problems but with different driving functions defined by $F(\boldsymbol{u}, h)$ in (14).

Let $\left(\boldsymbol{v}^{i}, q^{i}, \psi^{i}\right), i=1, \ldots, d$, be the steady-state solution to (19) when $u_{i}$ is observed at $\overline{\boldsymbol{x}}$ and $F_{\boldsymbol{u}}=\boldsymbol{e}^{i} \delta(\boldsymbol{x}-\overline{\boldsymbol{x}})$. These are solutions to the sensitivity problem. We will show that the adjoint solution and the variation $\delta \mathcal{F}$ of the inverse problem can be expressed in $\left(\boldsymbol{v}^{i}, q^{i}, \psi^{i}\right)$. The perturbation $\delta C$ is chosen such that $\delta \mathcal{F}<0$ in each step in the iterative solution of the inverse problem. Then the objective function $\mathcal{F}$ decreases stepwise toward the minimum.

It is shown in Appendix B that

$\left(\int_{\omega} \sum_{i=1}^{d} w_{i}(\overline{\boldsymbol{x}}) \boldsymbol{v}^{i} \mathrm{~d} \overline{\boldsymbol{x}}, \int_{\omega} \sum_{i=1}^{d} w_{i}(\overline{\boldsymbol{x}}) q^{i} \mathrm{~d} \overline{\boldsymbol{x}}, \int_{\omega} \sum_{i=1}^{d} w_{i}(\overline{\boldsymbol{x}}) \psi^{i} \mathrm{~d} \overline{\boldsymbol{x}}\right)$ is a solution of (19) with arbitrary weights $w_{i}(\overline{\boldsymbol{x}}), i=$ $1, \ldots, d$, when

$F_{\boldsymbol{u}}=\int_{\omega} \sum_{i=1}^{d} w_{i}(\overline{\boldsymbol{x}}) \boldsymbol{e}^{i} \delta(\boldsymbol{x}-\overline{\boldsymbol{x}}) \mathrm{d} \overline{\boldsymbol{x}}=\sum_{i=1}^{d} w_{i}(\overline{\boldsymbol{x}}) \boldsymbol{e}^{i}$.

When $C$ is perturbed, the first variation of the functional in (22) is

$\delta \mathcal{F}=\int_{\Gamma_{\mathrm{b}}} f(\mathbf{T} \boldsymbol{u}) \mathbf{T} \boldsymbol{u} \cdot \mathbf{T}\left(\int_{\omega} \sum_{i=1}^{d} w_{i}(\overline{\boldsymbol{x}}) \boldsymbol{v}^{i} \mathrm{~d} \overline{\boldsymbol{x}}\right) \delta C \mathrm{~d} \boldsymbol{x}$.

In the inverse problem in Petra et al. (2012),

$\mathcal{F}=\frac{1}{2} \int_{\omega}\left\|\boldsymbol{u}(\boldsymbol{x})-\boldsymbol{u}_{\mathrm{obs}}(\boldsymbol{x})\right\|^{2} \mathrm{~d} \boldsymbol{x}, \quad F_{\boldsymbol{u}}=\boldsymbol{u}(\boldsymbol{x})-\boldsymbol{u}_{\mathrm{obs}}(\boldsymbol{x})$.

The weights in (27) for the inverse problem are $w_{i}(\boldsymbol{x})=$ $u_{i}(\boldsymbol{x})-u_{\mathrm{obs}, i}(\boldsymbol{x})$. Let $\tilde{\boldsymbol{v}}$ denote a weighted sum of the solutions of the sensitivity problem $\boldsymbol{v}^{i}$ over the whole domain $\omega$ :

$\tilde{\boldsymbol{v}}(\boldsymbol{x})=\int_{\omega} \sum_{i=1}^{d}\left(u_{i}(\overline{\boldsymbol{x}})-u_{\mathrm{obs}, i}(\overline{\boldsymbol{x}})\right) \boldsymbol{v}^{i} \mathrm{~d} \overline{\boldsymbol{x}}$. 

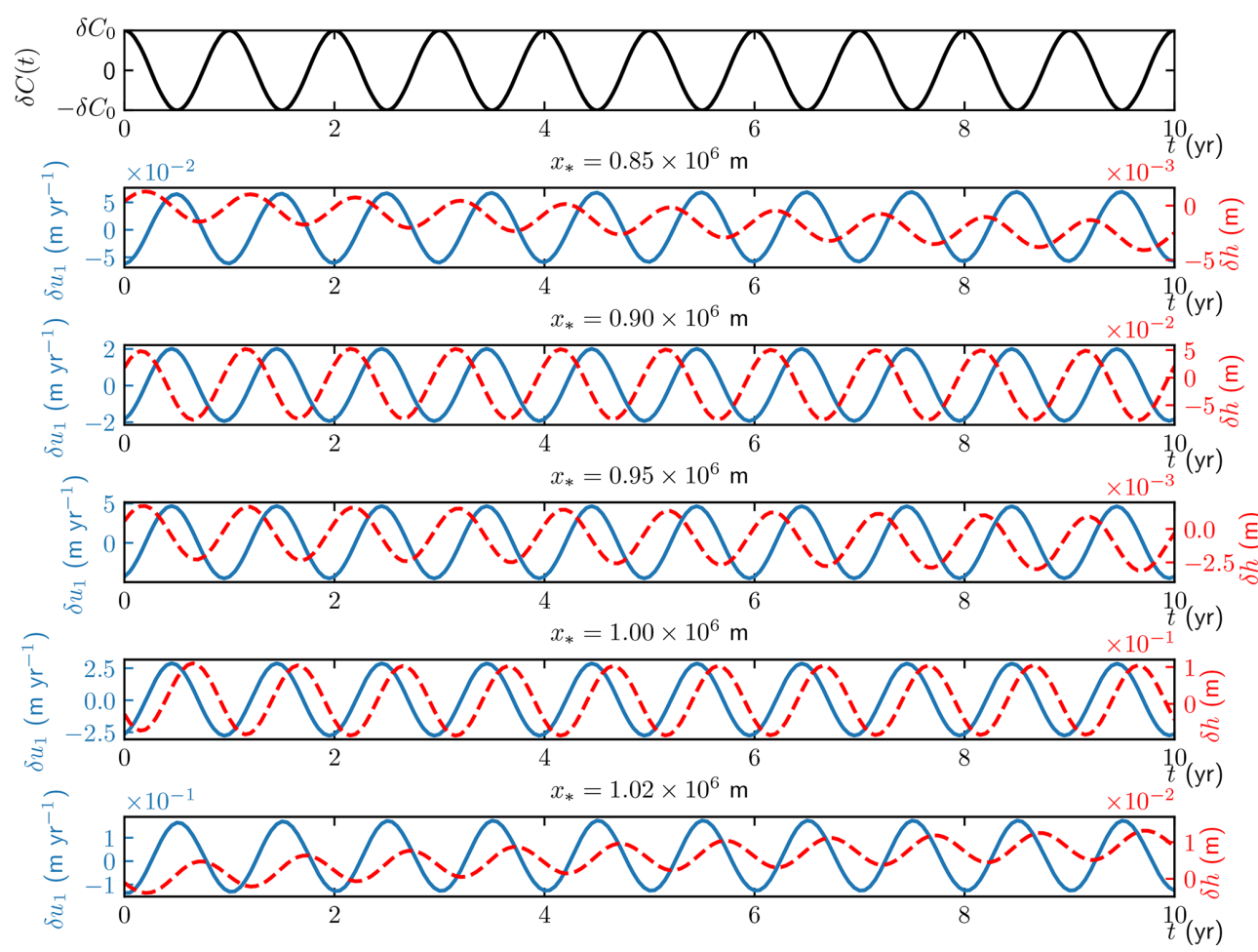

Figure 3. Observations at $x_{*}=0.85,0.9,0.95,1.0,1.02 \times 10^{6} \mathrm{~m}$ with FS in time $t \in[0,10]$ of $\delta u_{1}$ (solid blue) and $\delta h$ (dashed red) with perturbation $\delta C(t)=0.01 C \cos (2 \pi t)$ for $x \in[0.9,1.0] \times 10^{6} \mathrm{~m}$. Notice the different scales on the $y$ axes.

Then the effect of $\delta C$ on $\mathcal{F}$ in the inverse problem is by (28)

$\delta \mathcal{F}=\int_{\Gamma_{\mathrm{b}}} f(\mathbf{T} \boldsymbol{u}) \mathbf{T} \boldsymbol{u} \cdot \mathbf{T} \tilde{\boldsymbol{v}}(\boldsymbol{x}) \delta C \mathrm{~d} \boldsymbol{x}$.

The same construction of the solution is possible when $h_{\mathrm{obs}}$ is given. Then $d=1, F(h)=\frac{1}{2}\left(h-h_{\mathrm{obs}}\right)^{2}$, and $F_{h}=w=$ $h-h_{\text {obs. }}$.

We have investigated the relation between the sensitivity problem and the inverse problem. By solving $d$ sensitivity problems with $F_{\boldsymbol{u}}=\boldsymbol{e}^{i} \delta(\boldsymbol{x}-\overline{\boldsymbol{x}}), i=1, \ldots, d$, to obtain their adjoint solutions $\left(\boldsymbol{v}^{i}, q^{i}, \psi^{i}\right)$ and combine them with the weights $w_{i}$ from $F_{\boldsymbol{u}}$ in (29) for the inverse problem, the adjoint solution to the inverse problem is (30). This solution can then be inserted into (28) to evaluate the effect in $\mathcal{F}$ of a change in $C$ as in (31). In practice, if we are interested in solving the inverse problem and determine $\delta \mathcal{F}$ in (28) in order to iteratively compute the optimal solution with a gradient method, then we solve (19) directly with $F_{\boldsymbol{u}}=\boldsymbol{u}-\boldsymbol{u}_{\text {obs }}$ or $F_{h}=h-h_{\text {obs }}$ to obtain $\tilde{\boldsymbol{v}}$ without computing $d$ vectors $\boldsymbol{v}^{i}$. Taking $\delta C=-f(\mathbf{T} \boldsymbol{u}) \mathbf{T} \boldsymbol{u} \cdot \mathbf{T} \tilde{\boldsymbol{v}}$ in (31) guarantees that $\delta \mathcal{F}<0$.

\subsubsection{Steady-state solution to the adjoint elevation equation in two dimensions}

A further theoretical consideration shows that the solution $\psi$ to the adjoint elevation equation need not be computed to estimate perturbations in the velocity for a two-dimensional vertical ice sheet at a steady state. We show with the analytical solution in the FS model that the influence of $\psi$ is negligible. It is sufficient to solve the adjoint stress equation for $v$ to estimate the perturbation in the velocity.

The adjoint steady-state equation in a two-dimensional vertical ice in (19) is

$\left(u_{1} \psi\right)_{x}=F_{h}+\left(\boldsymbol{h} \psi+F_{\boldsymbol{u}}\right) \cdot \boldsymbol{u}_{z}, z=h, 0 \leq x \leq L$.

The velocity from the forward equation is $\boldsymbol{u}(x, z)=$ $\left(u_{1}, u_{3}\right)^{T}$, and the adjoint elevation $\psi$ satisfies the right boundary condition $\psi(L)=0$.

The analytical solution $\psi$ to (32) is derived in Appendix C. Let $g(x)=u_{1 z}(x)$ if $u_{1}$ is observed and let $g(x)=1$ if $h$ is observed. Then the adjoint solution is

$\psi(x)=\left\{\begin{array}{l}-\frac{g\left(x_{*}\right)}{u_{1}(x)} \exp \left(-\int_{x}^{x_{*}} \frac{\boldsymbol{h} \cdot \boldsymbol{u}_{z}(y)}{u_{1}(y)} \mathrm{d} y\right), \\ 0 \leq x \leq x_{*}, \\ 0, \quad x_{*}<x \leq L .\end{array}\right.$

So, this solution has a jump $-g\left(x_{*}\right) / u_{1}\left(x_{*}\right)$ at $x_{*}$.

With a small $\boldsymbol{h} \cdot \boldsymbol{u}_{z}(y) \approx 0$ in (33), an approximate solution is $\psi(x) \approx-g\left(x_{*}\right) / u_{1}(x)$. If $u_{1}$ is observed and $g(\boldsymbol{x})=$ $u_{1 z} \approx 0$, then $\psi(\boldsymbol{x}) \approx 0$ in (33) and $\psi \boldsymbol{h} \approx 0$ in (19). This is the case in the SSA of the FS model where $u_{1 z}(x)=0$ and in the SIA of the FS equations where $u_{1 z}(x, h)=0$ (Greve and Blatter, 2009; Hutter, 1983). When these approximations 
are accurate then $u_{1 z}$ will be small. Consequently, when $u_{1}$ is observed, the effect on $v$ in the adjoint stress equation of the solution $\psi$ of the adjoint advection equation in (19) is small. Solving only the adjoint stress equation for $\boldsymbol{v}$ as in Gillet-Chaulet et al. (2016), Isaac et al. (2015), and Petra et al. (2012) yields an adequate answer. Numerical solution in Cheng and Lötstedt (2020) of the adjoint FS equation (19) in two dimensions confirms that when $u_{1}$ is observed then $\psi(x)$ is negligible. The situation is different when $h$ is observed and $\psi \approx 1 / u_{1}\left(x_{*}\right)$ in (33).

\subsection{Adjoint equations based on SSA}

Starting from (9), a Lagrangian $\mathcal{L}$ of the SSA equations is defined, using the technique described and applied to the FS equations in Petra et al. (2012). The SSA Lagrangian in (A4) in Appendix A is similar to the FS Lagrangian in (15). By partial integration in $\mathcal{L}$ and evaluation at the forward solution $(\boldsymbol{u}, h)$, the adjoint SSA equations are obtained. Then, the effect of perturbed data at the ice base manifests itself at the ice surface as a perturbation $\delta \mathcal{L}$; for details, see Appendix A.

The adjoint SSA equations read

$$
\begin{aligned}
& \psi_{t}+\boldsymbol{u} \cdot \nabla \psi+2 \eta \mathbf{B}(\boldsymbol{u}): \mathbf{D}(\boldsymbol{v})-\rho g H \nabla \cdot \boldsymbol{v} \\
& \quad+\rho g \boldsymbol{v} \cdot \nabla b=F_{h}, \text { in } \omega, \quad 0 \leq t \leq T, \\
& \psi(\boldsymbol{x}, T)=0, \text { in } \omega, \quad \psi(\boldsymbol{x}, t)=0, \text { on } \gamma_{\mathrm{w}}, \\
& \nabla \cdot \tilde{\boldsymbol{\zeta}}(\boldsymbol{v})-C f(\boldsymbol{u})\left(\mathbf{I}+\mathbf{F}_{\omega}(\boldsymbol{u})\right) \boldsymbol{v}-H \nabla \psi \\
& \quad=-F_{\boldsymbol{u}}, \text { in } \omega, \\
& \boldsymbol{t} \cdot \tilde{\boldsymbol{\zeta}}(\boldsymbol{v}) \boldsymbol{n}=-C_{\gamma} f_{\gamma}(\boldsymbol{t} \cdot \boldsymbol{u})\left(1+F_{\gamma}(\boldsymbol{t} \cdot \boldsymbol{u})\right) \boldsymbol{t} \cdot \boldsymbol{v}, \\
& \quad \text { on } \gamma_{g}, \\
& \boldsymbol{t} \cdot \tilde{\boldsymbol{\zeta}}(\boldsymbol{v}) \boldsymbol{n}=0, \text { on } \gamma_{\mathrm{w}}, \\
& \boldsymbol{n} \cdot \boldsymbol{v}=0, \text { on } \gamma,
\end{aligned}
$$

where the adjoint viscosity $\tilde{\eta}$ and adjoint stress $\tilde{\boldsymbol{\zeta}}$ are (cf. 20 for the case of FS)

$$
\begin{aligned}
& \tilde{\boldsymbol{\eta}}(\boldsymbol{u})=\eta(\boldsymbol{u})\left(\mathcal{I}+\frac{1-n}{n \mathbf{B}(\boldsymbol{u}): \mathbf{D}(\boldsymbol{u})} \mathbf{B}(\boldsymbol{u}) \otimes \mathbf{D}(\boldsymbol{u})\right), \\
& \tilde{\boldsymbol{\zeta}}(\boldsymbol{v})=2 H \tilde{\boldsymbol{\eta}}(\boldsymbol{u}) \star \mathbf{B}(\boldsymbol{v}) .
\end{aligned}
$$

From (34) it is seen that the adjoint SSA equations have the same structure as the adjoint FS equation (19). There is one stress equation for the adjoint velocity $v$ and one equation for the Lagrange multiplier $\psi$ corresponding to the surface elevation equation in (9). However, the advection equation for $\psi$ in (34) depends on $\boldsymbol{v}$, implying a fully coupled system for $\boldsymbol{v}$ and $\psi$. Equations (34) are solved backward in time with a final condition on $\psi$ at $t=T$. As in (9), there is no time derivative in the stress equation. With a Weertman friction law, viz. $f(\boldsymbol{u})=\|\boldsymbol{u}\|^{m-1}$ and $f_{\gamma}(\boldsymbol{t} \cdot \boldsymbol{u})=|\boldsymbol{t} \cdot \boldsymbol{u}|^{m-1}$ (cf. also Appendix A1),

$\mathbf{F}_{\omega}(\boldsymbol{u})=\frac{m-1}{\boldsymbol{u} \cdot \boldsymbol{u}} \boldsymbol{u} \otimes \boldsymbol{u}, F_{\gamma}=m-1$.
If the friction coefficient $C$ at the ice base (both where it is grounded on bedrock $(C>0)$ and floating $(C=0))$ is changed by $\delta C$, if the bottom topography is changed by $\delta b$, and if the lateral friction coefficient $C_{\gamma}$ is changed by $\delta C_{\gamma}$, then it follows from Appendix A2 that the Lagrangian $\mathcal{L}$ is changed by (note that the weight in front of $\delta C$ in Eq. 36 is actually the same as in Eq. 22)

$$
\begin{aligned}
\delta \mathcal{L} & =\int_{0}^{T} \int_{\omega}(2 \eta \mathbf{B}(\boldsymbol{u}): \mathbf{D}(\boldsymbol{v})+\rho g \boldsymbol{v} \cdot \nabla h+\nabla \psi \cdot \boldsymbol{u}) \delta b \\
& -f(\boldsymbol{u}) \boldsymbol{u} \cdot \boldsymbol{v} \delta C \mathrm{~d} \boldsymbol{x} \mathrm{d} t-\int_{0}^{T} \int_{\gamma_{g}} f_{\gamma}(\boldsymbol{t} \cdot \boldsymbol{u}) \boldsymbol{t} \cdot \boldsymbol{u} \boldsymbol{t} \\
\cdot \boldsymbol{v} \delta C_{\gamma} \mathrm{d} s \mathrm{~d} t . &
\end{aligned}
$$

The same perturbations in $\delta C, \delta b$, and $\delta C_{\gamma}$ could be allowed for the FS equations in (22), but because the FS equations are more complicated than the SSA equations, the complexity of the derivation in the appendix and the expression for $\delta \mathcal{L}$ would increase considerably, which is why we refrain from considering them here.

Suppose that only $h$ is observed with $F_{h} \neq 0$ and $F_{\boldsymbol{u}}=0$ in (34). Then the adjoint elevation equation must be solved for $\psi \neq 0$ to have a $\boldsymbol{v} \neq \mathbf{0}$ in the adjoint stress equation and a perturbation in the Lagrangian in (36). The same result follows from the adjoint FS equations. If $F_{h} \neq 0$ and $F_{\boldsymbol{u}}=0$ in (19), then $\psi \neq 0$. Consequently, $\boldsymbol{v} \neq \mathbf{0}$ and a perturbation $\delta C$ will cause a perturbation $\delta \mathcal{L}$ in (22). The conclusion that the adjoint elevation equation must be solved if the surface elevation is observed is independent of the two ice models.

In a broader context, it is worth emphasizing that the adjoint equation derived in MacAyeal (1993) is identical to the stress equation in (34), if $H$ is constant, $\mathbf{F}_{\omega}=0$ (e.g., $m=1$ ), and $\tilde{\boldsymbol{\eta}}(\boldsymbol{u})=\eta(\boldsymbol{u})$.

\subsubsection{The two-dimensional adjoint solution}

The adjoint SSA equations in two vertical dimensions are derived from (34) in the same manner as (11), by letting $\psi$ and $v_{1}$ be independent of $y$ and setting $v_{2}=0$ and $C_{\gamma}=0$. To simplify the notation, we also let $v=v_{1}$. The adjoint equations for $v$ and $\psi$ follow either from simplifying (34) or from the Lagrangian and (11) and read as follows:

$$
\begin{aligned}
& \psi_{t}+u \psi_{x}+\left(\eta u_{x}-\rho g H\right) v_{x}+\rho g b_{x} v=F_{h}, \\
& 0 \leq t \leq T, 0 \leq x \leq L, \\
& \psi(x, T)=0, \psi(L, t)=0, \\
& \left(\frac{1}{n} \eta H v_{x}\right)_{x}-C m f(u) v-H \psi_{x}=-F_{\mathrm{u}}, \\
& v(0, t)=0, v(L, t)=0 .
\end{aligned}
$$

Note that the viscosity above is multiplied by a factor $1 / n$, $n>0$, which represents an extension of the adjoint SSA in 
MacAyeal (1993), where $n=1$ implicitly. The effect on the Lagrangian of perturbations $\delta b$ and $\delta C$ is obtained from (36):

$$
\begin{aligned}
\delta \mathcal{L} & =\int_{0}^{T} \int_{0}^{L}\left(\psi_{x} u+v_{x} \eta u_{x}+v \rho g h_{x}\right) \delta b \\
& -v f(u) u \delta C \mathrm{~d} x \mathrm{~d} t .
\end{aligned}
$$

The weights or sensitivity functions $w_{b}$ and $w_{C}$ multiplying $\delta b$ and $\delta C$ in the integral are defined by

$$
\begin{aligned}
& w_{b}(x, t)=\psi_{x} u+v_{x} \eta u_{x}+v \rho g h_{x}, \\
& w_{C}(x, t)=-v f(u) u .
\end{aligned}
$$

The steady-state solutions to the system (37) can be analyzed as in the forward equations in Sect. 2.2.2 after simplifications. The viscosity terms in (37) are often small and can hence be neglected, and we assume that the basal topography is characterized by a small spatial gradient $b_{x}$. The advantage resulting from these simplifications is that both the forward and adjoint equations can be solved analytically on a reduced computational domain where $x \in\left[0, x_{\mathrm{GL}}\right]$. The analytical approximations are less accurate close to the ice divide where some of the above assumptions are not valid. The adjoint equation (37) reduce to

$$
\begin{aligned}
& u \psi_{x}-\rho g H v_{x}=F_{h}, \quad 0 \leq x \leq x_{\mathrm{GL}}, \\
& \psi_{x}(0)=0, \psi\left(x_{\mathrm{GL}}\right)=0, \\
& -C m u^{m-1} v-H \psi_{x}=-F_{\mathrm{u}}, \\
& v(0)=0 .
\end{aligned}
$$

\subsubsection{The two-dimensional adjoint steady-state solution with velocity observation}

In this section, the analytical solution to the adjoint equation (40) is discussed. The derivation of the solution is detailed in Appendix E to Appendix F. It is here sufficient to recall that the solution given below is derived under the assumptions that $b_{x} \ll H_{x}$, and that $a$ and $C$ are constants.

For observations of $u$ at $x_{*}$,

$\mathcal{F}=\int_{0}^{L} u(x) \delta\left(x-x_{*}\right) \mathrm{d} x=u_{*}, F_{\mathbf{u}}=\delta\left(x-x_{*}\right), F_{h}=0$,

and the adjoint solutions are

$$
\begin{aligned}
& \psi(x)=\frac{C a^{m} x_{*}}{\rho g H_{*}^{m+3}}\left(x_{\mathrm{GL}}^{m}-x^{m}\right), x_{*}<x \leq x_{\mathrm{GL}}, \\
& \psi(x)=-\frac{1}{H_{*}}+\frac{C a^{m} x_{*}}{\rho g H_{*}^{m+3}}\left(x_{\mathrm{GL}}^{m}-x_{*}^{m}\right), 0 \leq x<x_{*}, \\
& v(x)=\frac{a x_{*}}{\rho g H_{*}^{m+3}} H^{m}, x_{*}<x \leq x_{\mathrm{GL}}, \\
& v(x)=0,0 \leq x<x_{*},
\end{aligned}
$$

where $\psi(x)$ and $v(x)$ have discontinuities at the observation point $x_{*}$. The perturbation of the Lagrangian (38) is with the Heaviside step function $\mathcal{H}(x)$ and the Dirac delta $\delta(x)$ (cf. Appendix F):

$$
\begin{aligned}
\delta u_{*}= & \delta \mathcal{L}=\int_{0}^{x_{\mathrm{GL}}} w_{b} \delta b+w_{C} \delta C \mathrm{~d} x \\
= & \int_{0}^{x_{\mathrm{GL}}}\left(\psi_{x} u+v_{x} \eta u_{x}+v \rho g h_{x}\right) \delta b-v u^{m} \delta C \mathrm{~d} x \\
= & \int_{x_{*}^{-}}^{x_{\mathrm{GL}}} \frac{a x_{*} H^{m}}{H_{*}^{m+3}}\left[(m+1) H_{x} \mathcal{H}\left(x-x_{*}\right)\right. \\
& \left.+H \delta\left(x-x_{*}\right)\right] \delta b-\frac{a x_{*}(a x)^{m}}{\rho g H_{*}^{m+3}} \delta C \mathrm{~d} x \\
= & \frac{u_{*} \delta b_{*}}{H_{*}}-\frac{u_{*}}{\rho g H_{*}^{m+2}} \int_{x_{*}}^{x_{\mathrm{GL}}} C(a x)^{m} \\
& \left((m+1) \frac{\delta b}{H}+\frac{\delta C}{C}\right) \mathrm{d} x,
\end{aligned}
$$

or, after scaling with $u_{*}$,

$$
\begin{gathered}
\frac{\delta u_{*}}{u_{*}}=\frac{\delta b_{*}}{H_{*}}-\frac{1}{\rho g H_{*}^{m+2}} \int_{x_{*}}^{x_{\mathrm{GL}}} C(a x)^{m} \\
\left((m+1) \frac{\delta b}{H}+\frac{\delta C}{C}\right) \mathrm{d} x .
\end{gathered}
$$

The relation in (43) between the relative perturbations $\delta b / H, \delta C / C$, and $\delta u / u$ can also be interpreted as a way to quantify the uncertainty in $u$. The uncertainty may be due to measurement errors in the topography $b$. For example, it is known that the true surface is in an interval $[b-\delta b, b+\delta b]$ around $b$ where, e.g., $\delta b=1 \mathrm{~m}$ or $\delta b$ has a normal distribution with zero mean and some variance. Such an uncertainty $\delta b$ in $b$ or similarly an uncertainty $\delta C$ in $C$ is propagated to an uncertainty $\delta u_{*}$ in $u$ at $x_{*}$ by (43); see Smith (2014). As an example, suppose that a $5 \%$ error in the surface velocity is acceptable, then the tolerable error in the topography could be $20-30 \mathrm{~m}$ with a $1000 \mathrm{~m}$ thick ice.

The perturbations $\delta u_{1 i}$ at discrete points $\boldsymbol{x}_{*, i}, i=1,2, \ldots$ due to perturbations $\delta C_{j}$ at discrete points $\boldsymbol{x}_{j}, j=1,2, \ldots$ are connected by a transfer matrix $\mathbf{W}_{C}$ in Cheng and Lötstedt (2020). The relation between $\delta u_{1 i}$ and $\delta C_{j}$ is for all $i$ and $j$ such that

$\delta u_{1 i}=\sum_{j} W_{C i j} \delta C_{j}$.

The elements $W_{C i j}$ of the transfer matrix correspond to quadrature coefficients in the discretization of the first integral in (42) with $\delta b=0$. The properties of $\mathbf{W}_{C}$ are examined 
numerically in Cheng and Lötstedt (2020). We conclude that certain perturbations of $C$ (not only highly oscillatory) are difficult to observe in $u_{1}$ at the surface. The same analysis is performed for the other combinations of $\delta b, \delta C$ and $\delta u_{1}, \delta h$.

Finally, let us comment on other approaches to investigate the sensitivity of surface data to changes in $b$ and $C$, e.g., using three linear models as in Gudmundsson (2008) and along a flow line at a steady state in Gudmundsson and Raymond (2008) with a linearized FS model with $n=1$ and $m=1$. In these papers, transfer functions for the perturbations from base to surface corresponding to our formulas (42) and (43) are derived by Fourier and Laplace analysis. The perturbations with long wavelength $\lambda$ and small wave number $k$ are propagated to the surface, but short wavelengths are effectively damped in Gudmundsson (2008). The transfer functions are utilized in Gudmundsson and Raymond (2008) to estimate how well basal data can be retrieved from surface data. Retrieval of basal slipperiness $C$ is possible for perturbations $\delta C$ of long wavelength and if the error in the basal topography $\delta b$ is small. Short wavelength perturbations $\delta b$ can be determined from surface data. The same conclusions as in Gudmundsson (2008) and Gudmundsson and Raymond (2008) can be drawn from our explicit expressions for the dependence of $\delta u_{*}$ and $\delta h_{*}$ on $\delta C$ and $\delta b$. For example, it follows from (45) that only $\delta C$ with a long wavelength is visible at the surface and that $\delta b$ also with a short wavelength affects $\delta u_{*}$ in (43). If $\delta b$ is small or zero in (43), then it is easier to determine the $\delta C$ that causes a certain $\delta u_{*}$.

The analytical adjoint solutions $\psi(x)$ and $v(x)$ in (41) of the MISMIP case in Fig. 2 with parameters in Table 1 at different $x_{*}$ positions are shown in Figs. 4a and 5a.

The weights $w_{b}$ and $w_{C}$ in (42) multiplying $\delta b$ and $\delta C$, defined in the same manner as in (38) and (39), are shown in Figs. 6a and 7a with the solutions $\psi$ and $v$ in Figs. $4 \mathrm{a}$ and 5a. The Dirac term is plotted as a vertical line at $x_{*}$ in Fig. 7a.

All perturbations in $C$ between $x_{*}$ and $x_{\mathrm{GL}}$ will result in a perturbation of the opposite sign in $u_{*}$ at the surface because $w_{C}<0$ in $\left(x_{*}, x_{\mathrm{GL}}\right)$ in Fig. 6a and (42). The same conclusion holds true for perturbations in $b$ because $w_{b}<0$ in $\left(x_{*}, x_{\mathrm{GL}}\right)$ in Fig. 7a, but an additional contribution is added from $\delta b$ at $x_{*}$ by the Dirac delta in $w_{b}$. A perturbation is less visible in $u$ the farther away from $x_{\mathrm{GL}}$ the observation point is since the amplitude of both $w_{C}$ and $w_{b}$ decays when $x_{*}$ decreases.

The following conclusions can be drawn from (42) and (43) and Figs. 6 and 7.

i The closer perturbations in basal friction are located to the grounding line, the larger perturbations of velocity will be observed at the surface. This is because the weight in front of $\delta C$ increases when $x_{*} \rightarrow x_{\mathrm{GL}}$, see Fig. 6, which in turn is an effect of the increasing velocity $u_{*}$ and the decreasing thickness $H_{*}$, as the grounding line is approached (see Fig. 2). Or, compactly expressed, $\delta C$ with support in $\left[x_{*}, x_{\mathrm{GL}}\right]$ will cause larger perturbations at the surface the closer $x_{*}$ is to $x_{\mathrm{GL}}$ and the closer $\delta C(x)$ is to $x_{\mathrm{GL}}$. The same conclusion is drawn in Cheng and Lötstedt (2020) with numerically computed SSA adjoint solutions.

ii Variations in the observed velocity $\delta u_{*}$ at the surface at observation point $x_{*}$ will include contributions from changes in the frictional parameter, $\delta C$, between $x_{*}$ and the grounding line $x_{\mathrm{GL}}$, as well as from changes in basal topography, $\delta b$, but it is impossible to disentangle their individual contributions to $\delta u_{*}$.

iii When the variation in ice thickness is small compared to the overall ice thickness, $H_{x} \ll H$, a small perturbation in basal topography $\delta b$ is directly visible in the surface velocity. This is because in such a case, $\delta u_{*} \approx$ $u_{*} \delta b_{*} / H_{*}$, and the main effect on $u_{*}$ from the perturbation $\delta b$ is localized at each $x_{*}$ (see Eq. 42 ).

iv For an unperturbed basal topography, two different perturbations of the friction coefficient will result in the same perturbation of the velocity. In other words, the perturbation $\delta C$ cannot be uniquely determined by one observation of $\delta u$. This follows if we let the perturbation of the friction coefficient be a constant $\delta C_{0} \neq 0$ in $\left[x_{0}, x_{1}\right] \in\left[x_{*}, x_{\mathrm{GL}}\right]$ and evaluate the integral in (42) to obtain

$$
\begin{aligned}
\delta u_{*} & =-\frac{u_{*}}{\rho g H_{*}^{m+2}} \int_{x_{0}}^{x_{1}}(a x)^{m} \delta C_{0} \mathrm{~d} x \\
& =-\frac{a^{m} u_{*}}{(m+1) \rho g H_{*}^{m+2}}\left(x_{1}^{m+1}-x_{0}^{m+1}\right) \delta C_{0} .
\end{aligned}
$$

The same $\delta u_{*}$ is observed with a constant perturbation in $\left[x_{2}, x_{3}\right] \in\left[x_{*}, x_{\mathrm{GL}}\right]$ with the amplitude $\delta C_{0}\left(x_{1}^{m+1}-\right.$ $\left.x_{0}^{m+1}\right) /\left(x_{3}^{m+1}-x_{2}^{m+1}\right)$.

$\mathrm{v}$ A rapidly varying friction coefficient at the base of the ice sheet will be difficult to identify by observing the velocity at the ice surface. In contrast, a smoothly varying friction coefficient at the base will be easily observable at the ice sheet surface. This is seen as follows: Perturb $C$ by $\delta C=\epsilon \cos \left(k x / x_{\mathrm{GL}}\right)$ in (42) for some wave number $k$, which determines the smoothness of the friction at the bedrock and amplitude $\epsilon$, and let $\delta b=0$ and $m=1$. The wavelength of the perturbation is $\lambda=2 \pi x_{\mathrm{GL}} / k$. When $k$ is small then the wavelength is long and the variation of $C+\delta C$ is smooth. When $k$ is large then the friction coefficient varies rapidly in $x$ with a short $\lambda$. 


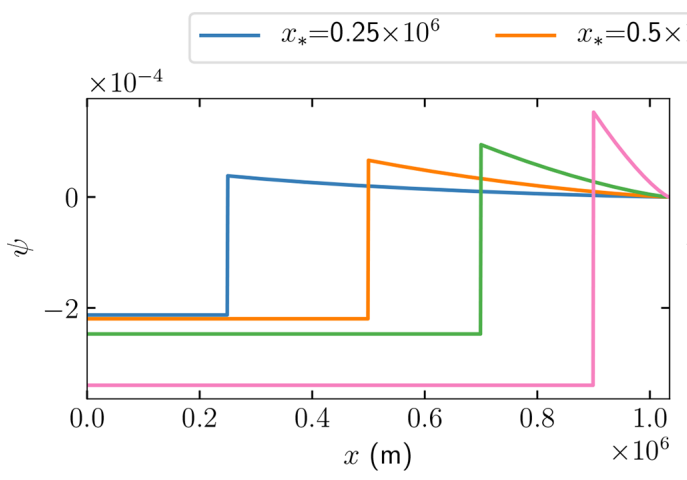

(a)

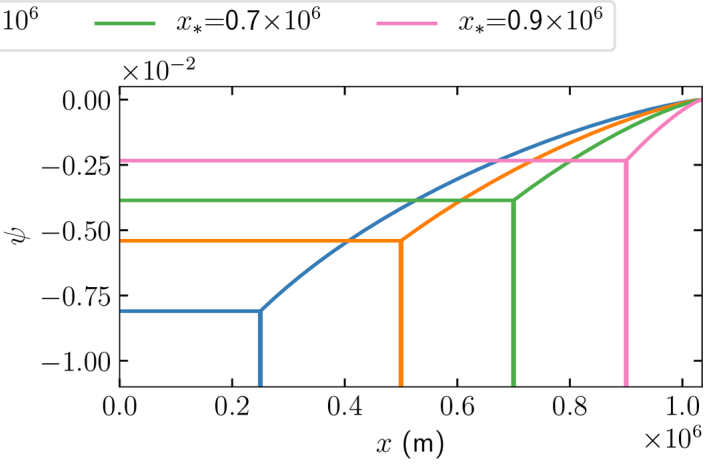

(b)

Figure 4. The analytical solutions of $\psi$ in (40) of the observations of (a) $u$ and (b) $h$ at different locations $x_{*}=0.25 \times 10^{6}, 0.5 \times 10^{6}, 0.7 \times 10^{6}$, and $0.9 \times 10^{6} \mathrm{~m}$.

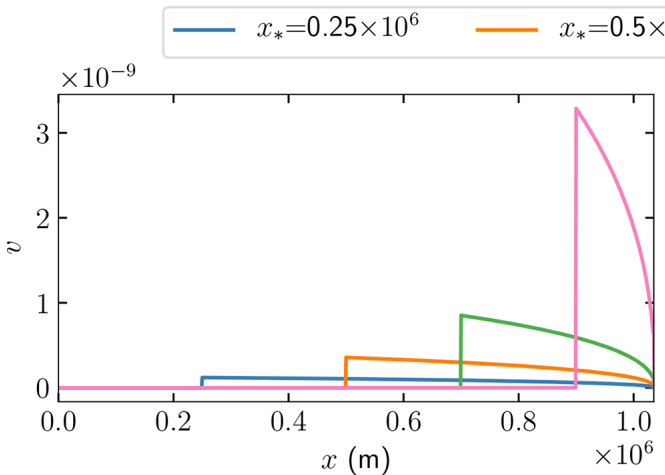

(a)

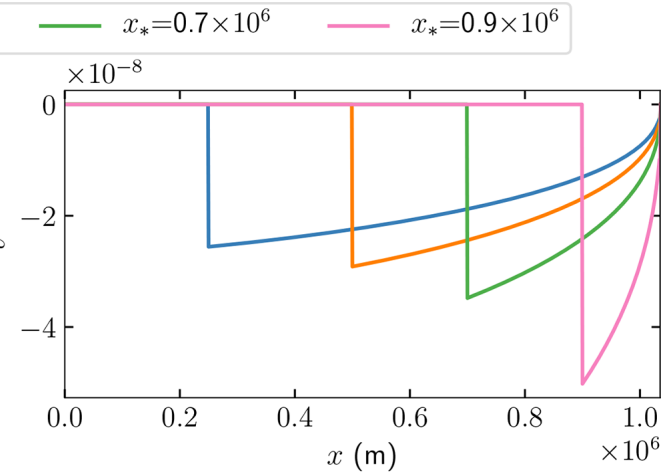

(b)

Figure 5. The analytical solutions of $v$ in (40) of the observations of (a) $u$ and (b) $h$ at different locations $x_{*}=0.25 \times 10^{6}, 0.5 \times 10^{6}, 0.7 \times 10^{6}$, and $0.9 \times 10^{6} \mathrm{~m}$.

The perturbation in the velocity is

$$
\begin{aligned}
\delta u_{*} & =-\int_{x_{*}}^{x_{\mathrm{GL}}} \epsilon \frac{a^{2} x_{*}}{\rho g H_{*}^{4}} x \cos \left(\frac{k x}{x_{\mathrm{GL}}}\right) \mathrm{d} x \\
& =-\epsilon \frac{a^{2} x_{*}}{\rho g H_{*}^{4}} \frac{x_{\mathrm{GL}}^{2}}{k}\left(\sin (k)-\frac{x_{*}}{x_{\mathrm{GL}}} \sin \left(\frac{k x_{*}}{x_{\mathrm{GL}}}\right)\right. \\
& \left.+\frac{1}{k}\left(\cos (k)-\cos \left(\frac{k x_{*}}{x_{\mathrm{GL}}}\right)\right)\right) .
\end{aligned}
$$

For a thin ice with a small $H_{*}$, a perturbation in $C$ is easier to observe at the surface than for a thick ice. When $k$ grows at the ice base, the amplitude of the perturbation at the ice surface decays as $1 / k$. Thus, the effect of high wave number perturbations of $C$ will be difficult to observe at the top of the ice, but smooth perturbations at the base will propagate to the surface. If $k$ is large and the surface velocity is of interest in a numerical simulation, then there is no reason to use a fine mesh at the base to resolve the fast variation in $C$ because it will not be visible at the top of the ice. How the damping de- pends on $\lambda$ in the FS equations is computed in Cheng and Lötstedt (2020).

vi A perturbation in the topography with long wavelength is easier to detect at the surface than a perturbation with short wavelength. If $\delta C=0$ and $b$ is perturbed by $\delta b=$ $\epsilon \cos \left(k x / x_{\mathrm{GL}}\right)$, then any perturbation at $x_{*}$ is propagated to the surface by $\frac{u_{*} \delta b_{*}}{H_{*}}$, which is the first term on the right-hand side of (42). The effect is larger if the ice is thin and moving fast. The integral term will behave in the same way as in (45), with mainly perturbations with small wave numbers and long wavelengths visible at the surface. 


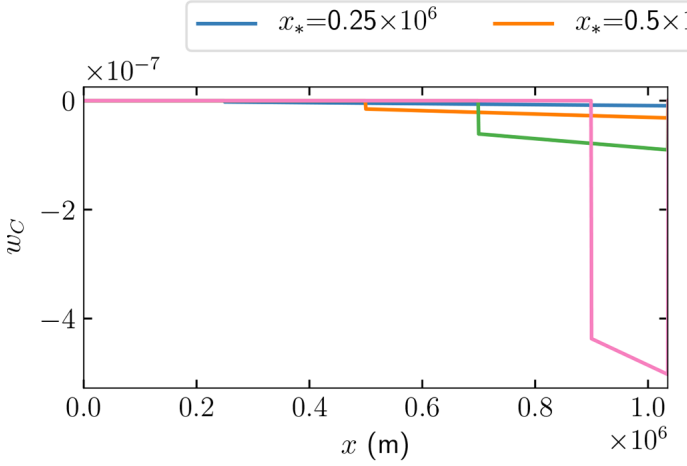

(a)

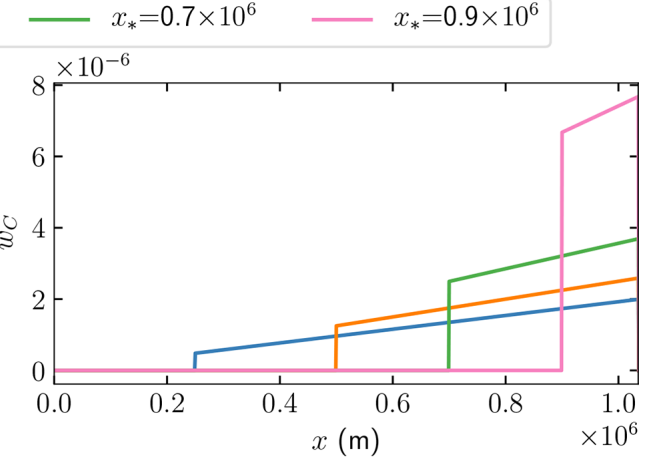

(b)

Figure 6. The analytical solution of the weights $w_{C}=-v u^{m}$ on $\delta C$ in (38) for (a) $u$ and (b) $h$ observed at $x_{*}=0.25 \times 10^{6}, 0.5 \times 10^{6}, 0.7 \times$ $10^{6}$, and $0.9 \times 10^{6} \mathrm{~m}$.

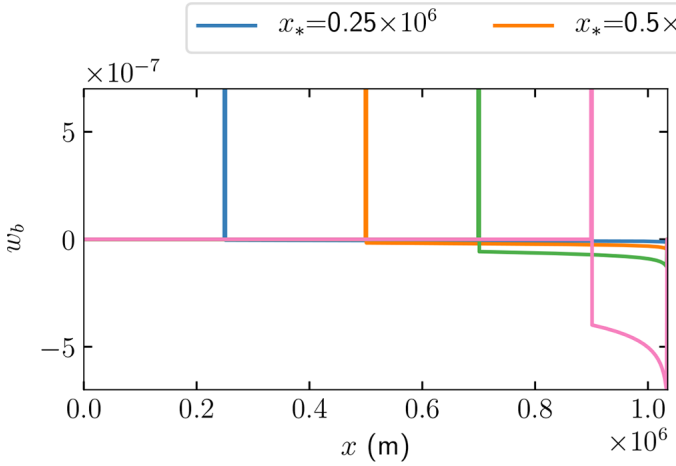

(a)

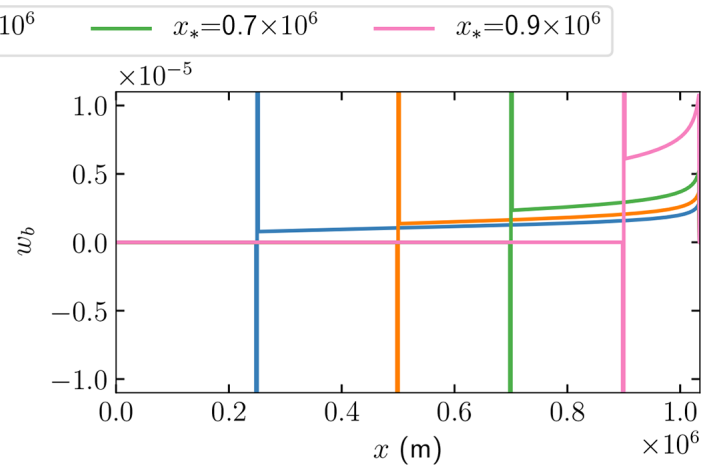

(b)

Figure 7. The analytical solution of weights $w_{b}=\psi_{x} u+v_{x} \eta u_{x}+v \rho g h_{x}$ on $\delta b$ in (38) for (a) $u$ and (b) $h$ observed at $x_{*}=0.25 \times 10^{6}, 0.5 \times$ $10^{6}, 0.7 \times 10^{6}$, and $0.9 \times 10^{6} \mathrm{~m}$.

\subsubsection{The two-dimensional adjoint steady-state solution with elevation observation}

In the case when $h$ is observed at $x_{*}$ and $F_{\mathrm{u}}=0$ and $F_{h}=$ $\delta\left(x-x_{*}\right)$, the expressions for $\psi$ and $v$ satisfying (40) are

$$
\begin{aligned}
\psi(x) & =-\frac{C a^{m-1}}{\rho g H_{*}^{m+1}}\left(x_{\mathrm{GL}}^{m}-x^{m}\right), x_{*}<x \leq x_{\mathrm{GL}}, \\
\psi(x) & =-\frac{C a^{m-1}}{\rho g H_{*}^{m+1}}\left(x_{\mathrm{GL}}^{m}-x_{*}^{m}\right), 0 \leq x<x_{*}, \\
v(x) & =-\frac{H^{m}}{\rho g H_{*}^{m+1}}, x_{*}<x \leq x_{\mathrm{GL}} \\
v(x) & =0,0 \leq x<x_{*} .
\end{aligned}
$$

The corresponding formulas when $u$ is observed are found in (41). There is a discontinuity at the observation point $x_{*}$ in $v(x)$ illustrated in Fig. 5b, but $\psi(x)$ is continuous in the solution of (40) and in Fig. 4b.

The second derivative term $\left(\frac{1}{n} \eta H v_{x}\right)_{x}$ is neglected in the simplified Eq. (40) but is of importance at $x_{*}$. A correction $\hat{\psi}$ of $\psi$ at $x_{*}$ in (46) is therefore introduced to satisfy $\left(\frac{1}{n} \eta H v_{x}\right)_{x}-H \hat{\psi}_{x}=0$. With $v_{x}\left(x_{*}\right)=-\delta\left(x-x_{*}\right) /\left(\rho g H_{*}\right)$, the correction is $\hat{\psi}(x)=-\delta\left(x-x_{*}\right) \eta_{*} /\left(n \rho g H_{*}\right)$. The solution $\psi$ is updated at each $x_{*}$ in Fig. $4 \mathrm{~b}$, with $\hat{\psi}$ as a vertical line representing the negative Dirac delta.

The perturbation in $h$ is as in (42) with $\psi$ and $v$ in (46) and the additional term $\hat{\psi}$ :

$$
\begin{aligned}
\frac{\delta h_{*}}{H_{*}}= & \int_{x_{*}^{-}}^{x_{\mathrm{GL}}}-\frac{u \eta_{*}}{n \rho g H_{*}^{2}} \delta_{x}\left(x-x_{*}\right) \delta b \mathrm{~d} x \\
& +\int_{x_{*}}^{x_{\mathrm{GL}}} \frac{C(a x)^{m}}{\rho g H_{*}^{m+2}}\left((m+1) \frac{\delta b}{H}+\frac{\delta C}{C}\right) \mathrm{d} x \\
= & \frac{a \eta_{*}}{n \rho g H_{*}^{2}}\left(x \frac{\delta b}{H}\right)_{x}\left(x_{*}\right)+\frac{1}{\rho g H_{*}^{m+2}} \int_{x_{*}}^{x_{\mathrm{GL}}} C(a x)^{m} \\
& \left((m+1) \frac{\delta b}{H}+\frac{\delta C}{C}\right) \mathrm{d} x,
\end{aligned}
$$

where $a(x \delta b / H)_{x}\left(x_{*}\right)=(u \delta b)_{x}\left(x_{*}\right)$ represents the $x$ derivative of $u \delta b$ evaluated at $x_{*}$. When $\delta b=0$ then $\delta u_{*}$ in 
(43) and $\delta h_{*}=\delta H_{*}$ in (47) satisfy $\delta u_{*} H_{*}=-\delta H_{*} u_{*}$ as in the integrated form of the advection equation in (12) and in (D1) in the Appendix.

As in (42), (47) is rewritten with the weights $w_{b}$ and $w_{C}$ in (39):

$$
\begin{aligned}
\delta h_{*} & =\int_{0}^{x_{\mathrm{GL}}}\left(\psi_{x} u+v_{x} \eta u_{x}+v \rho g h_{x}\right) \delta b-v u^{m} \delta C \mathrm{~d} x \\
& =\int_{0}^{x_{\mathrm{GL}}} w_{b} \delta b+w_{C} \delta C \mathrm{~d} x .
\end{aligned}
$$

These weights are shown in Figs. $6 \mathrm{~b}$ and $7 \mathrm{~b}$. The negative derivative of the Dirac delta is depicted in Fig. $7 \mathrm{~b}$ as a vertical line in the negative direction immediately followed by one in the positive direction.

The contribution from the integrals in (43) and (47) is identical except for the sign (compare $w_{C}$ in Fig. $6 \mathrm{a}$ and $\mathrm{b}$ and $w_{b}$ in Fig. 7a and b). The first term in (43) depends on $\delta b / H$, and the first term in (47) depends on the derivative of $a x \delta b / H=u \delta b$. The derivative of $u \delta b$ at $x_{*}$ directly affects the perturbation of $h$ at $x_{*}$. A perturbation of $b$ at the base is directly visible locally in $u$ at the surface, while the effect of $\delta C$ is non-local in the integral in (47). Because of the similarities between (43) and (47) and the left and right columns of Figs. 6 and 7, the conclusions (i), (ii), (iv), (v), and (vi) in Sect. 3.2.2 from (42) and (43) for $\delta u_{*}$ are valid also for $\delta h_{*}$ in (47).

\subsubsection{The two-dimensional time-dependent adjoint solution}

Finally, the time-dependent adjoint equation (37) is investigated. Equation (37) is solved numerically for the same MISMIP test case as Fig. 2 in Sect. 2.2.2 with the parameters in Table 1. As in Sect. 3.1.2, the friction coefficient $C$ has a seasonal variation (period $\tau 1$ year, where the beginning of the year is associated with winter) in the forward equation (11):

$C(x, t)=C_{0}(1+\kappa \cos (2 \pi t)), 0<\kappa<1$.

Apparently, $C$ has its highest value at $t=n, n=0,1,2, \ldots$, i.e., the winter, and its lowest value at $t=n+1 / 2$, i.e., the summer, as in Fig. 3. The amplitude of the variation in $C$ is set to $\kappa=0.5$, and the forward equation (11) is solved for 11 years. The GL is determined by the conditions in Sect. 2.2 and will move in time because of the variation in $C$. The topography $b$ is kept constant in time. Observations of $u$ and $h$ are made at $x_{*}=9 \times 10^{5} \mathrm{~m}$ for 0.1 year in the four seasons starting from the summer of the 10th year, e.g., in the summer $\left(t_{*}=9.5\right)$, the fall $\left(t_{*}=9.75\right)$, the winter $\left(t_{*}=10\right)$, and the spring $\left(t_{*}=10.25\right)$. The forward equation (11) is solved numerically from $t=0$ with the steady-state solution as initial data to the observation points $t=t_{*}$, and the adjoint equation (37) is solved from $t=t_{*}$ backward in time to $t=0$.
According to a convergence test, the time step is chosen to be 0.01 year and the spatial resolution is $10^{3} \mathrm{~m}$. A visual inspection of the computed solutions after halving the step sizes indicates that a sufficiently converged numerical solution has been reached.

Figure 8 shows the results for the adjoint weights $w_{C}(x, t)$ and $w_{b}(x, t)$ multiplying the perturbations $\delta C$ and $\delta b$, as defined in (38), for the observations of $u$ and $h$ at $x_{*}=9 \times 10^{5} \mathrm{~m}$ in all four seasons, where each column represents one season. The friction coefficient $C$ follows the seasonal variation in (49). Each row is one of the combinations of the weights $w_{C}$ and $w_{b}$ for the observations of $u$ and $h$. The time axis (or ordinate) in the figure follows the time direction in the forward problem (11). Most of the weights in space and time are negligible, implying that perturbations in those domains are not visible at $\left(x_{*}, t_{*}\right)$. Only $\delta C$ and $\delta b$ in a narrow interval around $x_{*}$ for $t$ in $\left[0, t_{*}\right]$ have an influence on $\delta u_{*}$ and $\delta h_{*}$. Therefore, we take a snapshot of the $x$ axis (or abscissa) with the width of $10^{5} \mathrm{~m}$ in space around $x_{*}$ in Fig. 8 . The weights oscillate in time because of the seasonal variation in the basal conditions in (49). A perturbation at the base is propagated to the $x_{*}$ position on the surface but with a possible delay in time. The earlier a perturbation in $C$ or $b$ takes place in the interval $\left[0, t_{*}\right)$, the smaller the effect of it is at $t_{*}$. After 5 years a perturbation can hardly be detected at the surface.

The temporal variations of the adjoint weights at $x_{*}$ in Fig. 8 are shown in Fig. 9 for the four seasons with four different colors. As expected, the weights vanish when $t>t_{*}$. In Fig. 9a and b, the perturbations $\delta C_{*}$ and $\delta b_{*}$ have a direct effect on $\delta u_{*}$ at $t_{*}$, where both $w_{C}\left(x_{*}, t_{*}\right)$ and $w_{b}\left(x_{*}, t_{*}\right)$ are negative. The same direct effect of $\delta C$ is found for $\delta u_{1 *}$ solving the FS equation (24) in Sect. 3.1.1. A change in $\delta C_{*}$ at the base is observed immediately as a change in $u$ at the surface. The effect of $\delta C$ on $\delta u_{*}$ for $t<t_{*}$ is weak in Fig. 9a; i.e., the memory of old perturbations is short. The largest effect of $\delta C$ on $\delta u_{*}$ and $\delta h_{*}$ appears with $t_{*}$ in the summer when $C$ is small in (49) (the blue lines in Fig. 9a and b).

However, when $h$ is observed, the effects of $\delta C_{*}$ and $\delta b_{*}$ are not visible directly because $w_{C *} \approx 0$ and $w_{b *} \approx 0$ in Fig. $9 \mathrm{c}$ and d. An intuitive explanation is that there is an immediate effect on the velocity, but there is a delay in $h$ since it is integrated in time from the velocity field. Additionally, the effects of $\delta C$ and $\delta b$ are difficult to separate, since the weight $w_{b}\left(x_{*}, t\right)$ has a shape similar to $w_{C}\left(x_{*}, t\right)$. The largest effect on $\delta h_{*}$ is from $\delta C$ in the summer due to the peaks in $w_{C}$ in Fig. 9c. For the same $\delta C$, the largest $\delta h_{*}$ is observed in the fall (orange), and then the second largest $\delta h_{*}$ is in the winter (green), followed by the spring observation (red). If $\delta h_{*}$ is observed in the fall and the time dependency is ignored, then the wrong conclusion is drawn that $\delta C$ in the fall has the strongest effect (but it is the summer perturbation). There is a delay in time between the perturbation and the observation of the effect in the surface elevation. The same shift in time is what we found in Sect. 3.1.1, (25), and Fig. 3 for the FS equations. 

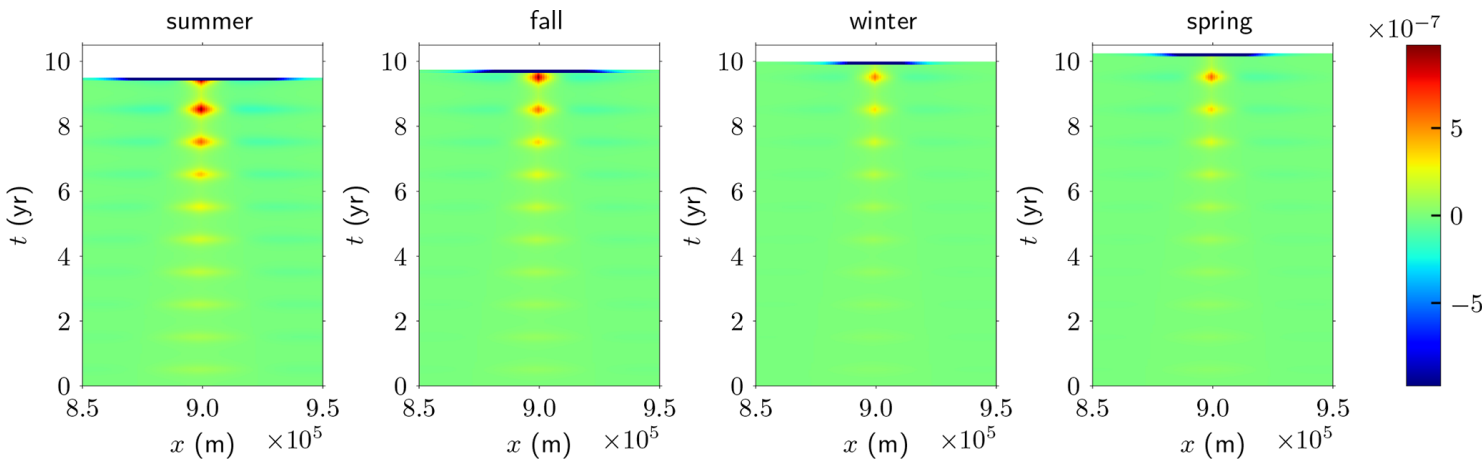

(a)
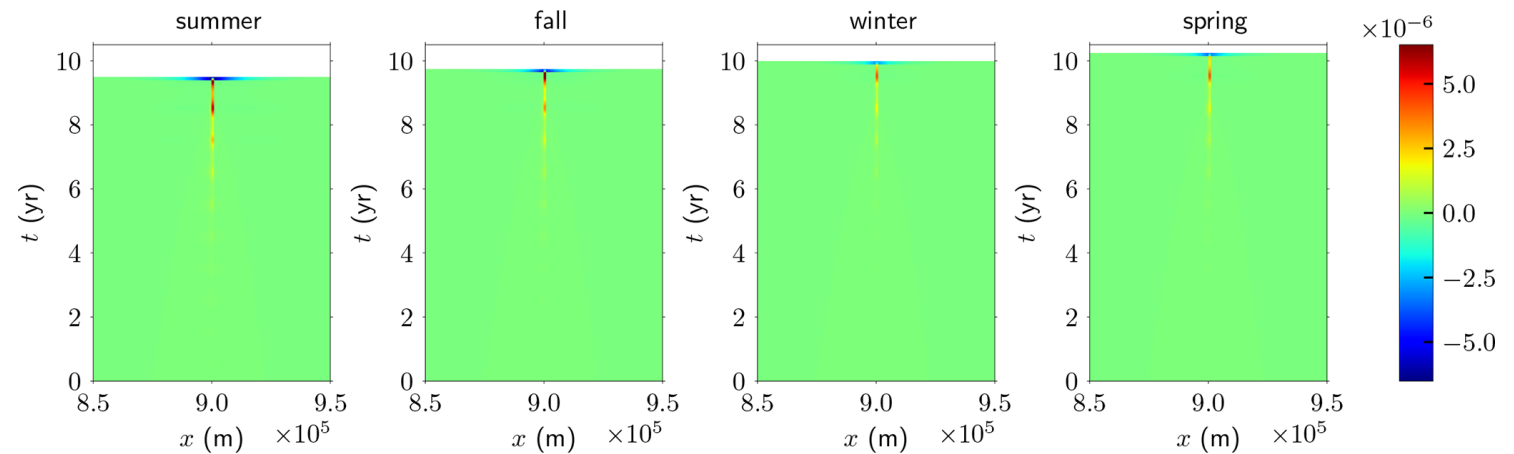

(b)
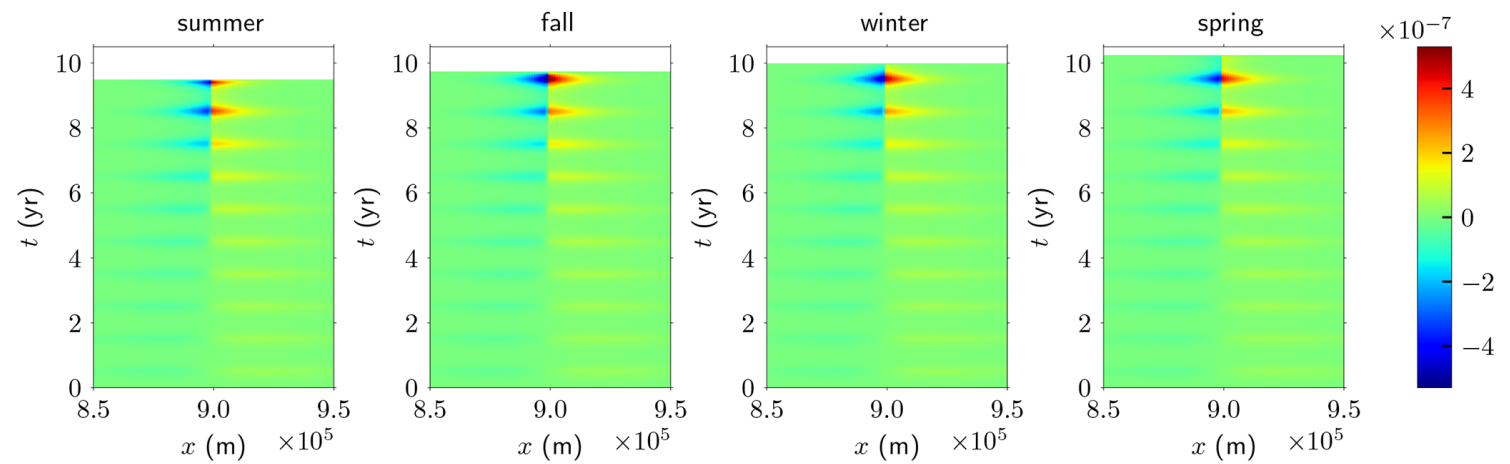

(c)
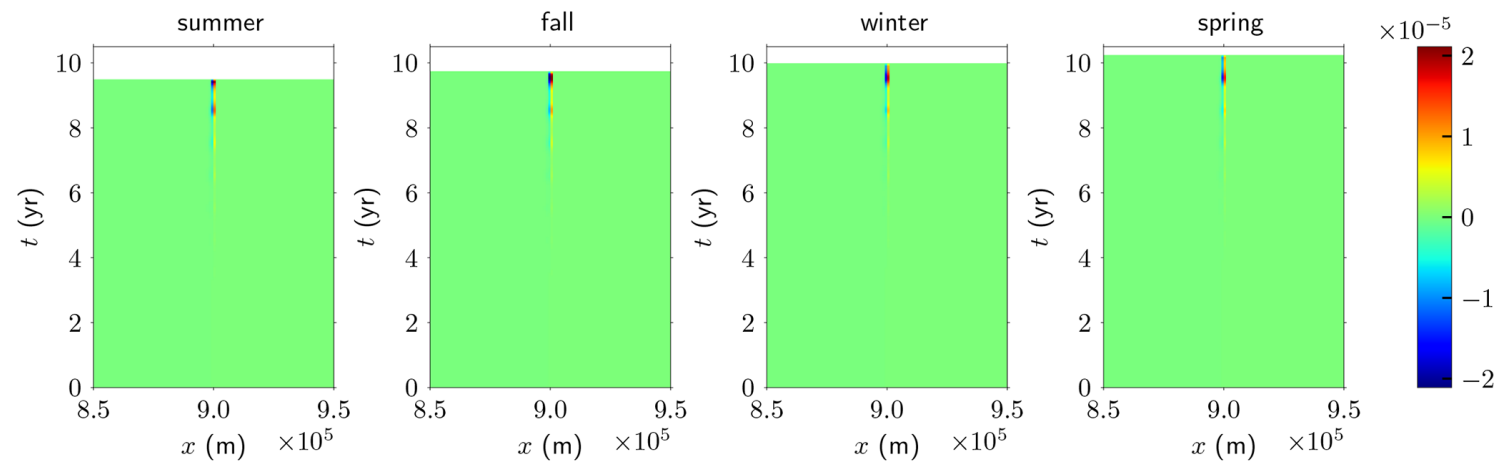

(d)

Figure 8. The adjoint weights for the observations at $x_{*}=9 \times 10^{5} \mathrm{~m}$ of the four seasons. (a) $w_{C}$ for the observation of $u$. (b) $w_{b}$ for the observation of $u$. (c) $w_{C}$ for the observation of $h$. (d) $w_{b}$ for the observation of $h$. 


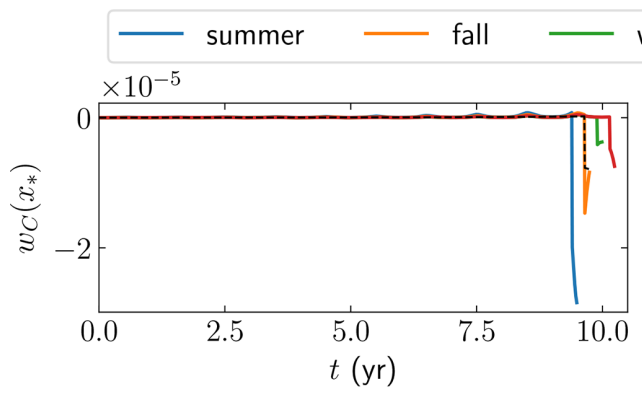

(a)

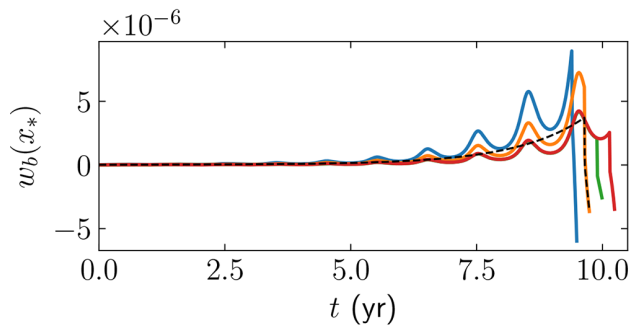

(b)

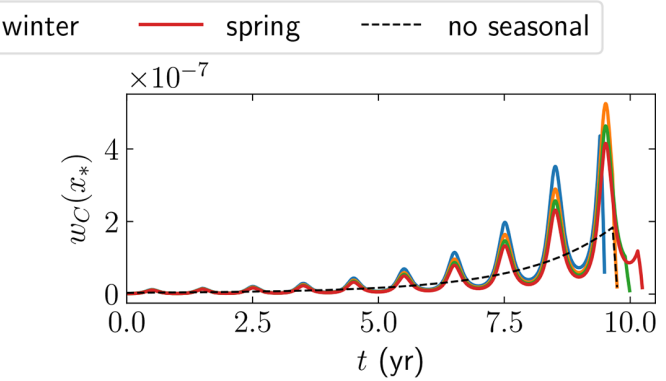

(c)

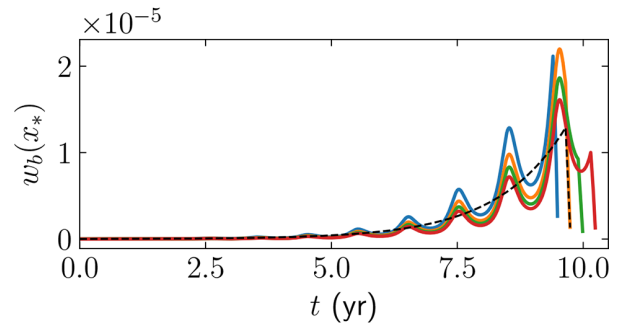

(d)

Figure 9. The adjoint weights at $x_{*}$ in the four seasons of the 10th year with seasonally varying friction coefficient. The black dashed line is a reference solution without seasonal variations which is observed at $t_{*}=9.75$. (a) $w_{C}$ for the observation of $u$. (b) $w_{b}$ for the observation of $u$. (c) $w_{C}$ for the observation of $h$. (d) $w_{b}$ for the observation of $h$.

A reference adjoint solution at $x_{*}$ observed during the fall season $\left(t_{*}=9.75\right)$ with time-independent $C$ and $b, \kappa=0$ in (49), as parameters in the forward equations is shown in black dashed lines in all the four panels of Fig. 9. The weight $w_{b}$ at $x_{*}$ for a constant $b$ is well approximated by $w_{b *} \exp (-(T-t) / \tau)$ in time with $\tau=1.4$ years for some $w_{b *}$ for the observation of both $u$ and $h$ in Fig. $9 \mathrm{~b}$ and d. For the weight $w_{C}$, the same exponential function holds with weight $w_{C *}$, but the time constant $\tau=1.8$ years for the observation of $h_{*}$ in Fig. 9c and $\tau=2.2$ years for the $u_{*}$ case.

Suppose that the temporal perturbation is oscillatory with frequency $f$ and located in space at $x_{*}$ with

$\delta C(x, t)=\delta C_{0} \cos (2 \pi f t) \delta\left(x-x_{*}\right)$.

A low-frequency $f$ with $f \ll 1$ corresponds to decennial or centennial variations and a high-frequency $f$ with $f \gg 1$ corresponds to diurnal or weekly variations. Then the perturbation in $h$ at $t=t_{*}$ is

$$
\begin{aligned}
\delta h_{*} & =\int_{0}^{t_{*}} w_{C *} \exp \left(-\left(t_{*}-t\right) / \tau\right) \delta C_{0} \cos (2 \pi f t) \mathrm{d} t \\
& =\left(\frac{\cos \left(2 \pi f t_{*}\right)+2 \pi \tau f \sin \left(2 \pi f t_{*}\right)-e^{-t_{*} / \tau}}{4 \pi^{2} \tau f^{2}+\tau^{-1}}\right) w_{C *} \delta C_{0}
\end{aligned}
$$

cf. (45). If the frequency is high, $f \gg 1$, then $\delta h_{*} \propto 1 / f$ and high-frequency perturbations are damped efficiently. At certain times of observation $t_{*}$ when $\sin \left(2 \pi f t_{*}\right)=0$, the damping is even stronger with $\delta h_{*} \propto 1 / f^{2}$. If the frequency is low, $f \ll 1$, then $\delta h_{*} \propto \tau$ and the change in $h_{*}$ is insensitive to the frequency. The same conclusions hold true for $\delta b$ where decennial perturbations seem more realistic.

\section{Conclusions}

The adjoint equations are derived in the FS and the SSA frameworks including time and the surface elevation equation. Time-dependent perturbations $\delta C$ and $\delta b$ in basal friction coefficient $C$ and basal topography $b$ are introduced, and their effect on observations of the velocity $\boldsymbol{u}$ and the surface elevation $h$ at the top surface of the grounded ice is studied. With the solution of the adjoint equations, we can determine the perturbation at a given point in space and time on the surface due to all basal perturbations. By solving the forward equations twice with $C$ and $C+\delta C$ or $b$ and $b+\delta b$, we can compute the perturbation in all points in space and time on the surface, e.g., $\delta u_{1}=u_{1}(C+\delta C)-u_{1}(C)$ in the first velocity component, for a given $\delta C$ or $\delta b$.

The perturbations in the observations are determined numerically in Cheng and Lötstedt (2020) either using the adjoint equations and their solutions in Sect. 3 or by solving the forward equations with unperturbed and perturbed parameters to obtain $\delta u_{*}$ and $\delta h_{*}$. The numerical solutions are compared to each other and to analytical solutions for SSA. The agreement is good in the comparisons.

In Sect. 3.1.3, a relation is established between the inverse problem (aiming to infer parameters from data) and the sensitivity problem (aiming to quantify the effect of variations in 
parameters): the same adjoint equations are solved. However, the forcing functions differ and are specific to the inverse problem and the sensitivity problem, respectively. Common to both problems is that the adjoint equations tell how perturbations in the parameters at the ice base are propagated to perturbations in the velocity and the elevation of the surface.

For steady-state problems, and in an FS setting where $\boldsymbol{u}$ is observed, we find (cf. Sect. 3.1.4) that the contribution of the solution of the adjoint elevation equation (32) is small and that it therefore suffices to solve only the adjoint stress equations - see, e.g., Gillet-Chaulet et al. (2016), Isaac et al. (2015), and Petra et al. (2012) - in order to be able to draw conclusions regarding perturbations of $\boldsymbol{u}$. For steady-state problems in a two-dimensional SSA setting for a vertical ice, (43), (47), and Figs. 6 and 7 show that the sensitivity of the velocity and elevation increases (because the velocity increases and the ice thickness decreases) as the observation point $x_{*}$ approaches the grounding line.

In this setting, a non-local effect of a perturbation in $C$ has been observed, in the sense that $\delta C(x)$ affects both $u\left(x_{*}\right)$ and $h\left(x_{*}\right)$ even if $x \neq x_{*}$, but a perturbation $\delta b$ in $b$ has a strong local effect concentrated at $x_{*}$. Nevertheless, the shapes of the two sensitivity functions (or weights) for $\delta b$ and $\delta C$ are very similar except for the neighborhood of $x_{*}$, which makes it difficult to separate their respective contribution in an observation. Different combinations of the perturbations in the basal friction and bedrock elevation can produce the same effect on the velocity and surface elevation changes at one observation point.

In the inverse problems based on time-dependent simulations of FS and SSA, it is necessary to include the adjoint elevation equation. If the perturbations in the basal conditions are time dependent and $h$ is observed (see Figs. 3, 9c, and d), then time cannot be ignored in the inversion. If time dependence is ignored, wrong conclusions concerning the conditions at the ice base may be drawn from observations of $h$, in both the FS and the SSA model. In the time-dependent solution of SSA, a perturbation of the basal condition at $x_{*}$ has the strongest impact at $x_{*}$ on the surface, possibly with a time delay. Such a time delay occurs when a perturbation at the ice base is visible at the surface in $h$, but in $u$ it is observed immediately (Fig. 9). The effect of a perturbation disappears more quickly the older the perturbation is.

Perturbations in the friction coefficient at the base observed in the surface velocity determined by SSA are damped inversely proportional to the wave number and the frequency of the perturbations in (45) and (50), thus making very oscillatory perturbations in space and time difficult to register at the ice sheet surface. In such a case, there is no need to have a fine mesh and a small time step in a numerical solution to resolve the rapid oscillations in $C$ at the base. 


\section{Appendix A: Derivation of the adjoint equations}

\section{A1 Adjoint viscosity and friction in SSA}

The adjoint viscosity $\tilde{\boldsymbol{\eta}}(\boldsymbol{u})$ in SSA in (20) is derived as follows. The SSA viscosity for $\boldsymbol{u}$ and $\boldsymbol{u}+\delta \boldsymbol{u}$ is

$$
\begin{aligned}
& \eta(\boldsymbol{u}+\delta \boldsymbol{u}) \approx \eta(\boldsymbol{u})\left(1+\frac{1-n}{2 n \hat{\eta}}\left(\left(2 u_{1 x}+u_{2 y}\right) \delta u_{1 x}\right.\right. \\
& +\frac{1}{2}\left(u_{1 y}+u_{2 x}\right) \delta u_{2 x}+\left(2 u_{2 y}+u_{1 x}\right) \delta u_{2 y} \\
& \left.\left.+\frac{1}{2}\left(u_{1 y}+u_{2 x}\right) \delta u_{1 y}\right)\right) .
\end{aligned}
$$

Determine $\mathcal{B}(\boldsymbol{u})$ such that

$\varrho(\boldsymbol{u}, \delta \boldsymbol{u}) \mathbf{B}(\boldsymbol{u})=\mathcal{B}(\boldsymbol{u}) \star \mathbf{B}(\delta \boldsymbol{u})$.

First note that

$$
\begin{aligned}
& \mathbf{B}(\boldsymbol{u}): \mathbf{D}(\delta \boldsymbol{u})=(\mathbf{D}(\boldsymbol{u})+\nabla \cdot \boldsymbol{u I}): \mathbf{D}(\delta \boldsymbol{u})=\mathbf{D}(\boldsymbol{u}): \mathbf{D}(\delta \boldsymbol{u}) \\
& \quad+(\nabla \cdot \boldsymbol{u})(\nabla \cdot \delta \boldsymbol{u})=\mathbf{D}(\boldsymbol{u}):(\mathbf{B}(\delta \boldsymbol{u})-\nabla \cdot \delta \boldsymbol{u} \mathbf{I}) \\
& \quad+(\nabla \cdot \boldsymbol{u})(\nabla \cdot \delta \boldsymbol{u})=\mathbf{D}(\boldsymbol{u}): \mathbf{B}(\delta \boldsymbol{u}) .
\end{aligned}
$$

Then use the $\star$ operator to define $\mathcal{B}$ :

$$
\begin{aligned}
& \frac{1-n}{2 n \hat{\eta}} \sum_{k l} B_{k l}(\boldsymbol{u}) D_{k l}(\delta \boldsymbol{u}) B_{i j}(\boldsymbol{u}) \\
& =\frac{1-n}{2 n \hat{\eta}} \sum_{k l} D_{k l}(\boldsymbol{u}) B_{k l}(\delta \boldsymbol{u}) B_{i j}(\boldsymbol{u}) \\
& =\sum_{k l} \mathcal{B}_{i j k l}(\boldsymbol{u}) D_{k l}(\delta \boldsymbol{u})=(\mathcal{B} \star D)_{i j}
\end{aligned}
$$

Thus, let

$\mathcal{B}_{i j k l}=\frac{1-n}{2 n \hat{\eta}} B_{i j}(\boldsymbol{u}) D_{k l}(\boldsymbol{u})$,

$\tilde{\eta}_{i j k l}(\boldsymbol{u})=\eta(\boldsymbol{u})\left(\mathcal{I}_{i j k l}+\mathcal{B}_{i j k l}(\boldsymbol{u})\right)$,

or in tensor form

$$
\mathcal{B}=\frac{1-n}{n \mathbf{B}(\boldsymbol{u}): \mathbf{D}(\boldsymbol{u})} \mathbf{B}(\boldsymbol{u}) \otimes \mathbf{D}(\boldsymbol{u}),
$$$$
\tilde{\boldsymbol{\eta}}(\boldsymbol{u})=\eta(\boldsymbol{u})(\mathcal{I}+\mathcal{B}) \text {. }
$$

Replacing $\mathbf{B}$ in (A2) by $\mathbf{D}$, we obtain the adjoint FS viscosity in (20).

The adjoint friction in SSA in $\omega$ and at $\gamma_{g}$ in (34) with a Weertman law is derived as in the adjoint FS equations (19) and (20). Then in $\omega$ with $\boldsymbol{\xi}=\boldsymbol{u}, \boldsymbol{\zeta}=\boldsymbol{v}, c=C$, and $\mathbf{F}=\mathbf{F}_{\omega}$ and at $\gamma_{g}$ with $\boldsymbol{\xi}=\boldsymbol{t} \cdot \boldsymbol{u}, \boldsymbol{\zeta}=\boldsymbol{t} \cdot \boldsymbol{v}, c=C_{\gamma}, f=f_{\gamma}$, and $\mathbf{F}=$ $F_{\gamma}$, we arrive at the adjoint friction term $c f(\xi)(\mathbf{I}+\mathbf{F}(\xi)) \zeta$, where

$\mathbf{F}(\boldsymbol{\xi})=\frac{m-1}{\xi \cdot \xi} \boldsymbol{\xi} \otimes \xi$

\section{A2 Adjoint equations in SSA}

The Lagrangian for the SSA equations is with the adjoint variables $\psi, \boldsymbol{v}, q$

$$
\begin{aligned}
& \mathcal{L}\left(\boldsymbol{u}, h ; \boldsymbol{v}, \psi ; b, C_{\gamma}, C\right)= \\
& \int_{0}^{T} \int_{\omega} F(\boldsymbol{u}, h)+\psi\left(h_{t}+\nabla \cdot(\boldsymbol{u} H)-a\right) \mathrm{d} \boldsymbol{x} \mathrm{d} t \\
& +\int_{0}^{T} \int_{\omega} \boldsymbol{v} \cdot \nabla \cdot(2 H \eta \mathbf{B}(\boldsymbol{u}))-C f(\boldsymbol{u}) \boldsymbol{v} \cdot \boldsymbol{u}-\rho g H \boldsymbol{v} \cdot \nabla h \mathrm{~d} \boldsymbol{x} \mathrm{d} t \\
& =\int_{0}^{T} \int_{\omega} F(\boldsymbol{u}, h)+\psi\left(h_{t}+\nabla \cdot(\boldsymbol{u} H)-a\right) \mathrm{d} \boldsymbol{x} \mathrm{d} t \\
& +\int_{0}^{T} \int_{\omega}-2 H \eta(\boldsymbol{u})(\mathbf{D}(\boldsymbol{v}): \mathbf{D}(\boldsymbol{u})+\nabla \cdot \boldsymbol{u} \nabla \cdot \boldsymbol{v}) \\
& -C f(\boldsymbol{u}) \boldsymbol{v} \cdot \boldsymbol{u}-\rho g H \boldsymbol{v} \cdot \nabla h \mathrm{~d} \boldsymbol{x} \mathrm{d} t-\int_{0}^{T} \int_{\gamma_{g}} C_{\gamma} f_{\gamma}(\boldsymbol{t} \cdot \boldsymbol{u}) \boldsymbol{t} \\
& \cdot \boldsymbol{u t} \cdot \boldsymbol{v} \mathrm{d} s \mathrm{~d} t
\end{aligned}
$$

after partial integration and using the boundary conditions. The perturbed SSA Lagrangian is split into the unperturbed Lagrangian and three integrals:

$\mathcal{L}\left(\boldsymbol{u}+\delta \boldsymbol{u}, h+\delta h ; \boldsymbol{v}+\delta \boldsymbol{v}, \psi+\delta \psi ; b+\delta b, C_{\gamma}+\delta C_{\gamma}\right.$,

$$
\begin{aligned}
& C+\delta C)=\int_{0}^{T} \int_{\omega} F(\boldsymbol{u}+\delta \boldsymbol{u}, h+\delta h) \mathrm{d} \boldsymbol{x} \mathrm{d} t+\int_{0} \int_{\omega}(\psi+\delta \psi) \\
& \left(h_{t}+\delta h_{t}+\nabla \cdot((\boldsymbol{u}+\delta \boldsymbol{u})(H+\delta H))-a\right) \mathrm{d} \boldsymbol{x} \mathrm{d} t \\
& \quad+\int_{0}^{T} \int_{\omega}-2(H+\delta H) \eta(\boldsymbol{u}+\delta \boldsymbol{u}) \mathbf{D}(\boldsymbol{v}+\delta \boldsymbol{v}): \mathbf{B}(\boldsymbol{u}+\delta \boldsymbol{u}) \\
& -(C+\delta C) f(\boldsymbol{u}+\delta \boldsymbol{u})(\boldsymbol{u}+\delta \boldsymbol{u}) \cdot(\boldsymbol{v}+\delta \boldsymbol{v})-\rho g(H+\delta H) \\
& \nabla(h+\delta h) \cdot(\boldsymbol{v}+\delta \boldsymbol{v}) \mathrm{d} \boldsymbol{x} \mathrm{d} t-\int_{0}^{T} \int_{\gamma_{g}}\left(C_{\gamma}+\delta C_{\gamma}\right) f_{\gamma} \\
& (\boldsymbol{t} \cdot(\boldsymbol{u}+\delta \boldsymbol{u})) \boldsymbol{t} \cdot(\boldsymbol{u}+\delta \boldsymbol{u}) \boldsymbol{t} \cdot(\boldsymbol{v}+\delta \boldsymbol{v}) \mathrm{d} s \mathrm{~d} t \\
& =\mathcal{L}\left(\boldsymbol{u}, h ; \boldsymbol{v}, \psi ; b, C_{\gamma}, C\right)+I_{1}+I_{2}+I_{3} .
\end{aligned}
$$

The perturbation in $\mathcal{L}$ is

$\delta \mathcal{L}=I_{1}+I_{2}+I_{3}$. 
Terms of order 2 or more in $\delta \mathcal{L}$ are neglected. Then the first term in $\delta \mathcal{L}$ satisfies

$$
\begin{aligned}
I_{1} & =\int_{0}^{T} \int_{\omega} F(\boldsymbol{u}+\delta \boldsymbol{u}, h+\delta h)-F(\boldsymbol{u}, h) \mathrm{d} \boldsymbol{x} \mathrm{d} t \\
& =\int_{0}^{T} \int_{\omega} F_{\boldsymbol{u}} \delta \boldsymbol{u}+F_{h} \delta h \mathrm{~d} \boldsymbol{x} \mathrm{d} t .
\end{aligned}
$$

Using partial integration, Gauss' formula, and the initial and boundary conditions on $\boldsymbol{u}$ and $H$ and $\psi(\boldsymbol{x}, T)=0, \boldsymbol{x} \in \omega$, and $\psi(\boldsymbol{x}, t)=0, \boldsymbol{x} \in \gamma_{\mathrm{w}}$, in the second integral we have

$$
\begin{aligned}
I_{2} & =\int_{0}^{T} \int_{\omega} \delta \psi\left(h_{t}+\nabla \cdot(\boldsymbol{u} H)-a\right) \\
& +\psi\left(\delta h_{t}+\nabla \cdot(\delta \boldsymbol{u} H)+\nabla \cdot(\boldsymbol{u} \delta H)\right) \mathrm{d} \boldsymbol{x} \mathrm{d} t \\
& =\int_{0}^{T} \int_{\omega} \delta \psi\left(h_{t}+\nabla \cdot(\boldsymbol{u} H)-a\right) \mathrm{d} \boldsymbol{x} \mathrm{d} t+\int_{0}^{T} \int_{\omega} \\
& -\psi_{t} \delta h-H \nabla \psi \cdot \delta \boldsymbol{u}-\nabla \psi \cdot \boldsymbol{u} \delta H \mathrm{~d} \boldsymbol{x} \mathrm{d} t .
\end{aligned}
$$

The first integral after the second equality vanishes since $h$ is a weak solution and $I_{2}$ is

$$
\begin{aligned}
I_{2} & =\int_{0}^{T} \int_{\omega}-\left(\psi_{t}+\boldsymbol{u} \cdot \nabla \psi\right) \delta h-H \nabla \psi \cdot \delta \boldsymbol{u} \\
& +\boldsymbol{u} \cdot \nabla \psi \delta b \mathrm{~d} \boldsymbol{x} \mathrm{d} t .
\end{aligned}
$$

Using the weak solution of (9), the adjoint viscosity (35), (A2), the friction coefficient (A3), Gauss' formula, and the boundary conditions, as well as neglecting the second-order terms, the third and fourth integrals in (A5) are

$$
\begin{aligned}
I_{3}= & I_{31}+I_{32}, \\
I_{31}= & \int_{0}^{T} \int_{\omega}-2(H+\delta H) \eta(\boldsymbol{u}+\delta \boldsymbol{u}) \mathbf{D}(\boldsymbol{v}+\delta \boldsymbol{v}) \\
& : \mathbf{B}(\boldsymbol{u}+\delta \boldsymbol{u})-(C+\delta C) f(\boldsymbol{u}+\delta \boldsymbol{u})(\boldsymbol{u}+\delta \boldsymbol{u}) \\
& \cdot(\boldsymbol{v}+\delta \boldsymbol{v}) \\
& -\rho g(H+\delta H) \nabla(h+\delta h) \cdot(\boldsymbol{v}+\delta \boldsymbol{v})) \mathrm{d} \boldsymbol{x} \mathrm{d} t \\
& -\int_{0}^{T} \int_{\gamma}\left(C_{\gamma}+\delta C_{\gamma}\right) f_{\gamma}(\boldsymbol{t} \cdot(\boldsymbol{u}+\delta \boldsymbol{u})) \boldsymbol{t} \\
& \cdot(\boldsymbol{u}+\delta \boldsymbol{u}) \boldsymbol{t} \cdot(\boldsymbol{v}+\delta \boldsymbol{v}) \mathrm{d} s \mathrm{~d} t \\
= & I_{311}+I_{312}-I_{313},
\end{aligned}
$$

where

$$
\begin{aligned}
I_{311}= & \int_{0}^{T} \int_{\omega}-2 H \mathbf{D}(\boldsymbol{v}):(\eta(\boldsymbol{u}+\delta \boldsymbol{u}) \mathbf{B}(\boldsymbol{u}+\delta \boldsymbol{u}))+2 H \mathbf{D}(\boldsymbol{v}) \\
& :(\eta(\boldsymbol{u}) \mathbf{B}(\boldsymbol{u})) \mathrm{d} \boldsymbol{x} \mathrm{d} t \\
= & \int_{0}^{T} \int_{\omega}-2 H \mathbf{D}(\boldsymbol{v}):(\tilde{\boldsymbol{\eta}}(\boldsymbol{u}) \star \mathbf{B}(\delta \boldsymbol{u})) \mathrm{d} \boldsymbol{x} \mathrm{d} t \\
I_{312}= & \int_{0}^{T} \int_{\omega_{g}}-\delta C f(\boldsymbol{u}) \boldsymbol{u} \cdot \boldsymbol{v} \mathrm{d} \boldsymbol{x} \mathrm{d} t+\int_{0}^{T} \int_{\omega_{g}}-C(f(\boldsymbol{u}+\delta \boldsymbol{u}) \boldsymbol{v} \\
& \cdot(\boldsymbol{u}+\delta \boldsymbol{u})-f(\boldsymbol{u}) \boldsymbol{v} \cdot \boldsymbol{u}) \mathrm{d} \boldsymbol{x} \mathrm{d} t \\
= & \int_{0}^{T} \int_{\omega_{g}}-\delta C f(\boldsymbol{u}) \boldsymbol{u} \cdot \boldsymbol{v}+C f(\boldsymbol{u})\left(\mathbf{I}+\mathbf{F}_{\omega}(\boldsymbol{u})\right) \delta \boldsymbol{u} \cdot \boldsymbol{v} \mathrm{d} \boldsymbol{x} \mathrm{d} t \\
I_{313}= & \int_{0}^{T} \int_{\gamma_{g}}\left(C_{\gamma}+\delta C_{\gamma}\right)\left(f_{\gamma}(\boldsymbol{t} \cdot(\boldsymbol{u}+\delta \boldsymbol{u})) \boldsymbol{t} \cdot \boldsymbol{v} \boldsymbol{t} \cdot(\boldsymbol{u}+\delta \boldsymbol{u})\right. \\
& \left.-f_{\gamma}(\boldsymbol{t} \cdot \boldsymbol{u}) \boldsymbol{t} \cdot \boldsymbol{v} \boldsymbol{t} \cdot \boldsymbol{u}\right) \mathrm{d} s \mathrm{~d} t \\
& \int_{T} \int_{0}\left(C_{\gamma}+\delta C_{\gamma}\right)\left(f_{\gamma}(\boldsymbol{t} \cdot \boldsymbol{u}) \boldsymbol{t} \cdot \boldsymbol{u} \boldsymbol{t} \cdot \boldsymbol{v}+C_{\gamma} f_{\gamma}(\boldsymbol{t} \cdot \boldsymbol{u})\right. \\
& \left(\mathbf{I}+\mathbf{F}_{\gamma}(\boldsymbol{t} \cdot \boldsymbol{u})\right) \boldsymbol{t} \cdot \delta \boldsymbol{u} \boldsymbol{t} \cdot \boldsymbol{v} \mathrm{d} s \mathrm{~d} t \\
I_{32}= & \int_{0}^{T} \int_{\omega}-\rho g H \nabla h \cdot \boldsymbol{v}-2 \eta \mathbf{D}(\boldsymbol{v}): \mathbf{B}(\boldsymbol{u}) \delta H-\rho g \nabla h \\
& \cdot \boldsymbol{v} \delta H-\rho g H \boldsymbol{v} \cdot \nabla \delta h \mathrm{~d} \boldsymbol{x} \mathrm{d} t \\
& \int_{0}^{T} \int_{\omega}-\rho g H \nabla h \cdot \boldsymbol{v}-(2 \eta \mathbf{D}(\boldsymbol{v}): \mathbf{B}(\boldsymbol{u})+\rho g \nabla h \cdot \boldsymbol{v}) \\
& \\
& \\
&
\end{aligned}
$$


Collecting all the terms in (A7), (A9), and (A10), the first variation of $\mathcal{L}$ is

$$
\begin{aligned}
\delta \mathcal{L}= & I_{1}+I_{2}+I_{3} \\
= & \int_{0}^{T} \int_{\omega} F_{\boldsymbol{u}} \delta \boldsymbol{u}-2 H D(\boldsymbol{v}):(\tilde{\boldsymbol{\eta}}(\boldsymbol{u}) \star \mathbf{B}(\delta \boldsymbol{u}))-H \nabla \psi \\
& \cdot \delta \boldsymbol{u} \mathrm{d} \boldsymbol{x} \mathrm{d} t-\int_{0}^{T} \int_{\omega_{g}} C f(\boldsymbol{u})\left(\mathbf{I}+\mathbf{F}_{\omega}(\boldsymbol{u})\right) \boldsymbol{v} \cdot \delta \boldsymbol{u} \mathrm{d} \boldsymbol{x} \mathrm{d} t \\
& -\int_{0}^{T} \int_{\gamma_{g}} C_{\gamma} f_{\gamma}(\boldsymbol{t} \cdot \boldsymbol{u})\left(\mathbf{I}+\mathbf{F}_{\gamma}(\boldsymbol{t} \cdot \boldsymbol{u})\right) \boldsymbol{t} \cdot \boldsymbol{v} \boldsymbol{t} \cdot \delta \boldsymbol{u} \mathrm{d} s \mathrm{~d} t \\
& -\int_{0}^{T} \int_{\gamma_{g}} \delta C_{\gamma} f_{\gamma}(\boldsymbol{t} \cdot \boldsymbol{u}) \boldsymbol{t} \cdot \boldsymbol{u} \boldsymbol{t} \cdot \boldsymbol{v} \mathrm{d} s \mathrm{~d} t \\
& +\int_{0}^{T} \int_{\omega}\left(F_{h}-\left(\psi_{t}+\boldsymbol{u} \cdot \nabla \psi+2 \eta \mathbf{D}(\boldsymbol{v}): \mathbf{B}(\boldsymbol{u})\right.\right. \\
& -\rho g \nabla b \cdot \boldsymbol{v}+\rho g H \nabla \cdot \boldsymbol{v})) \delta h \mathrm{~d} \boldsymbol{x} \mathrm{d} t \\
& +\int_{0}^{T} \int_{\omega}-\delta C f(\boldsymbol{u}) \boldsymbol{v} \cdot \boldsymbol{u}+(2 \eta \mathbf{D}(\boldsymbol{v}): \mathbf{B}(\boldsymbol{u}) \\
& +\rho g \nabla h \cdot \boldsymbol{v}+\boldsymbol{u} \cdot \nabla \psi) \delta b \mathrm{~d} \boldsymbol{x} \mathrm{d} t .
\end{aligned}
$$

The forward solution $\left(\boldsymbol{u}^{*}, p^{*}, h^{*}\right)$ and adjoint solution $\left(\boldsymbol{v}^{*}, q^{*}, \psi^{*}\right)$ satisfying (9) and (34) are inserted into (A4) resulting in

$$
\begin{gathered}
\mathcal{L}\left(\boldsymbol{u}^{*}, p^{*} ; \boldsymbol{v}^{*}, q^{*} ; h^{*}, \psi^{*} ; b, C_{\gamma}, C\right) \\
=\int_{0}^{T} \int_{\omega} F\left(\boldsymbol{u}^{*}, h^{*}\right) \mathrm{d} \boldsymbol{x} \mathrm{d} t .
\end{gathered}
$$

Then (A12) yields the variation in $\mathcal{L}$ in (A13) with respect to perturbations $\delta b, \delta C_{\gamma}$, and $\delta C$ in $b, C_{\gamma}$, and $C$ :

$$
\begin{aligned}
\delta \mathcal{L}= & \int_{0}^{T} \int_{\omega}\left(2 \eta \mathbf{D}\left(\boldsymbol{v}^{*}\right): \mathbf{B}\left(\boldsymbol{u}^{*}\right)+\rho g \nabla h^{*} \cdot \boldsymbol{v}^{*}\right. \\
& \left.+\boldsymbol{u}^{*} \cdot \nabla \psi^{*}\right) \delta b \mathrm{~d} \boldsymbol{x} \mathrm{d} t-\int_{0}^{T} \int_{\gamma_{g}} \delta C_{\gamma} f_{\gamma}\left(\boldsymbol{t} \cdot \boldsymbol{u}^{*}\right) \boldsymbol{t} \\
& \cdot \boldsymbol{u}^{*} \boldsymbol{t} \cdot \boldsymbol{v}^{*} \mathrm{~d} s \mathrm{~d} t-\int_{0}^{T} \int_{\omega} \delta C f\left(\boldsymbol{u}^{*}\right) \boldsymbol{v}^{*} \cdot \boldsymbol{u}^{*} \mathrm{~d} \boldsymbol{x} \mathrm{d} t .
\end{aligned}
$$

\section{A3 Adjoint equations in FS}

The FS Lagrangian is as in (15):

$$
\begin{gathered}
\mathcal{L}(\boldsymbol{u}, p, h ; \boldsymbol{v}, q, \psi ; C)=\int_{0}^{T} \int_{\Gamma_{\mathrm{s}}} F(\boldsymbol{u}, h)+\psi\left(h_{t}+\boldsymbol{h}\right. \\
\left.\cdot \boldsymbol{u}-a_{\mathrm{S}}\right) \mathrm{d} \boldsymbol{x} \mathrm{d} t+\int_{0}^{T} \int_{\omega} \int_{b}^{h}-\boldsymbol{v} \cdot(\nabla \cdot \boldsymbol{\sigma}(\boldsymbol{u}, p))-q \nabla \\
\cdot \boldsymbol{u}-\rho \boldsymbol{g} \cdot \boldsymbol{v} \mathrm{d} \boldsymbol{x} \mathrm{d} t=\int_{0}^{T} \int_{\Gamma_{\mathrm{S}}} F(\boldsymbol{u}, h)+\psi\left(h_{t}+\boldsymbol{h}\right. \\
\cdot \boldsymbol{u}-a) \mathrm{d} \boldsymbol{x} \mathrm{d} t+\int_{0}^{T} \int_{\omega}^{h} \int_{b}^{h} 2 \eta(\boldsymbol{u}) \mathbf{D}(\boldsymbol{v}): \mathbf{D}(\boldsymbol{u})-p \nabla \\
\cdot \boldsymbol{v}-q \nabla \cdot \boldsymbol{u}-\rho \boldsymbol{g} \cdot \boldsymbol{v} \mathrm{d} \boldsymbol{x} \mathrm{d} t+\int_{0}^{T} \int_{\Gamma_{\mathrm{b}}} C f(\mathbf{T} \boldsymbol{u}) \mathbf{T} \boldsymbol{u}
\end{gathered}
$$

$\cdot \mathbf{T} v \mathrm{~d} \boldsymbol{x} \mathrm{d} t$.

In the same manner as in (A5), the perturbed FS Lagrangian is

$$
\begin{gathered}
\mathcal{L}(\boldsymbol{u}+\delta \boldsymbol{u}, p+\delta p ; \boldsymbol{v}+\delta \boldsymbol{v}, q+\delta q ; h+\delta h, \psi+\delta \psi ; \\
C+\delta C)=\mathcal{L}(\boldsymbol{u}, p, h ; \boldsymbol{v}, q, \psi ; C)+I_{1}+I_{2}+I_{3} .
\end{gathered}
$$

Terms of order 2 or more in $\delta \boldsymbol{u}, \delta \boldsymbol{v}$, and $\delta h$ are neglected. The first integral $I_{1}$ in (A16) is

$$
\begin{aligned}
I_{1} & =\int_{0}^{T} \int_{\Gamma_{\mathrm{S}}} F(\boldsymbol{u}(\boldsymbol{x}, h+\delta h, t)+\delta \boldsymbol{u}, h+\delta h) \\
& -F(\boldsymbol{u}(\boldsymbol{x}, h, t), h) \mathrm{d} \boldsymbol{x} \mathrm{d} t=\int_{0}^{T} \int_{\Gamma_{\mathrm{s}}} F_{\boldsymbol{u}}\left(\delta \boldsymbol{u}+\boldsymbol{u}_{z} \delta h\right) \\
& +F_{h} \delta h \mathrm{~d} \boldsymbol{x} \mathrm{d} t .
\end{aligned}
$$

Partial integration, the conditions $\psi(\boldsymbol{x}, T)=0$ and $\psi(\boldsymbol{x}, t)=$ 0 at $\Gamma_{\mathrm{s}}$, and the fact that $h$ is a weak solution simplify the second integral:

$$
\begin{aligned}
I_{2} & =\int_{0}^{T} \int_{\Gamma_{\mathrm{s}}} \delta \psi\left(h_{t}+\boldsymbol{h} \cdot \boldsymbol{u}-a_{\mathrm{S}}\right)+\psi\left(\delta h_{t}+\boldsymbol{u} \cdot \delta \boldsymbol{h}\right. \\
& \left.+\boldsymbol{u}_{z} \cdot \boldsymbol{h} \delta h+\boldsymbol{h} \cdot \delta \boldsymbol{u}\right) \mathrm{d} \boldsymbol{x} \mathrm{d} t=\int_{0}^{T} \int_{\Gamma_{\mathrm{s}}} \delta \psi\left(h_{t}+\boldsymbol{h} \cdot \boldsymbol{u}\right. \\
& \left.-a_{\mathrm{s}}\right) \mathrm{d} \boldsymbol{x} \mathrm{d} t+\int_{0}^{T} \int_{\Gamma_{\mathrm{s}}}\left(-\psi_{t}-\nabla \cdot(\boldsymbol{u} \psi)\right. \\
& \left.+\boldsymbol{h} \cdot \boldsymbol{u}_{z} \psi\right) \delta h+\boldsymbol{h} \cdot \delta \boldsymbol{u} \psi \mathrm{d} \boldsymbol{x} \mathrm{d} t .
\end{aligned}
$$


Define $\Xi, \xi$, and $\Upsilon$ to be

$$
\begin{aligned}
\Theta(\boldsymbol{u}, p ; \boldsymbol{v}, q ; C)= & 2 \eta(\boldsymbol{u}) \mathbf{D}(\boldsymbol{v}): \mathbf{D}(\boldsymbol{u})-p \nabla \cdot \boldsymbol{v} \\
& -q \nabla \cdot \boldsymbol{u}-\rho \boldsymbol{g} \cdot \boldsymbol{v}, \\
\theta(\boldsymbol{u} ; \boldsymbol{v} ; C)= & C f(\mathbf{T} \boldsymbol{u}) \mathbf{T} \boldsymbol{u} \cdot \mathbf{T} \boldsymbol{v}, \\
\Upsilon(\boldsymbol{u}, p ; \boldsymbol{v}, q)= & -\boldsymbol{v} \cdot(\nabla \cdot \boldsymbol{\sigma}(\boldsymbol{u}, p))-q \nabla \\
& \cdot \boldsymbol{u}-\rho \boldsymbol{g} \cdot \boldsymbol{v} .
\end{aligned}
$$

Then a weak solution, $(\boldsymbol{u}, p)$, for any $(\boldsymbol{v}, q)$ satisfying the boundary conditions, fulfills

$$
\begin{aligned}
& \int_{0}^{T} \int_{\omega} \int_{b}^{h} \Theta(\boldsymbol{u}, p ; \boldsymbol{v}, q ; C) \mathrm{d} \boldsymbol{x} \mathrm{d} t-\int_{0}^{T} \int_{\Gamma_{\mathrm{b}}} \theta(\boldsymbol{u} ; \boldsymbol{v} ; C) \\
& \mathrm{d} \boldsymbol{x} \mathrm{d} t=0 .
\end{aligned}
$$

The third integral in (A16) is

$$
\begin{aligned}
I_{3}= & I_{31}+I_{32}, \\
I_{31}= & \int_{0}^{T} \int_{\omega} \int_{b}^{h} \Theta(\boldsymbol{u}+\delta \boldsymbol{u}, p+\delta p ; \boldsymbol{v}+\delta \boldsymbol{v}, q+\delta q ; \\
& C+\delta C) \mathrm{d} \boldsymbol{x} \mathrm{d} t-\int_{0}^{T} \int_{\Gamma_{\mathrm{b}}} \theta(\boldsymbol{u}+\delta \boldsymbol{u} ; \boldsymbol{v}+\delta \boldsymbol{v} ; \\
& C+\delta C) \mathrm{d} \boldsymbol{x} \mathrm{d} t, \\
I_{32}= & \int_{0}^{T} \int_{\omega}^{h+\delta h} \int_{h}^{\Upsilon} \Upsilon(\boldsymbol{u}, p ; \boldsymbol{v}, q) \mathrm{d} \boldsymbol{x} \mathrm{d} t .
\end{aligned}
$$

The integral $I_{31}$ is expanded as in (A10) and (A11) or Petra et al. (2012) using the weak solution, Gauss' formula, and the definitions of the adjoint viscosity and adjoint friction coefficient in Appendix A1. When $b<z<h$ we have $\Upsilon(\boldsymbol{u}, p ; \boldsymbol{v}, q)=0$. If $\Upsilon$ is extended smoothly in the positive $z$ direction from $z=h$, then with $z \in[h, h+\delta h]$ for some constant $c>0$ we have $|\Upsilon| \leq c \delta h$. Therefore,

$$
\begin{aligned}
& \left|\int_{h}^{h+\delta h(x, t)} \Upsilon(\boldsymbol{u}, p ; \boldsymbol{v}, q) d z\right| \leq \int_{h}^{h+\delta h(x, t)} \sup |\Upsilon| d z \\
& \leq c\left|\delta h(x, t)^{2}\right|,
\end{aligned}
$$

and the bound on $I_{32}$ in (A21) is

$\left|I_{32}\right| \leq c t|\omega| \max |\delta h(x, t)|^{2}$,

where $|\omega|$ is the area of $\omega$. This term is a second variation in $\delta h$ which is neglected and $I_{3}=I_{31}$.
The first variation of $\mathcal{L}$ is then

$$
\begin{aligned}
\delta \mathcal{L}= & I_{1}+I_{2}+I_{3} \\
= & \int_{0}^{T} \int_{\Gamma_{\mathrm{s}}}\left(F_{\boldsymbol{u}}+\psi \boldsymbol{h}\right) \cdot \delta \boldsymbol{u} \mathrm{d} \boldsymbol{x} \mathrm{d} t+\int_{0}^{T} \int_{\Gamma_{\mathrm{s}}}\left(F_{h}+F_{\boldsymbol{u}} \boldsymbol{u}_{z}\right. \\
& \left.-\left(\psi_{t}+\nabla \cdot(\boldsymbol{u} \psi)-\boldsymbol{h} \cdot \boldsymbol{u} \psi\right)\right) \delta h \mathrm{~d} \boldsymbol{x} \mathrm{d} t \\
& +\int_{0}^{T} \int_{\omega} \int_{b}^{h} 2 \mathbf{D}(\boldsymbol{v}):(\tilde{\boldsymbol{\eta}}(\boldsymbol{u}) \star \mathbf{D}(\delta \boldsymbol{u}))-\delta p \nabla \cdot \boldsymbol{v} \\
& -q \nabla \cdot \delta \boldsymbol{u} \mathrm{d} \boldsymbol{x} \mathrm{d} t+\int_{0}^{T} \int_{\Gamma_{\mathrm{b}}} C f(\mathbf{T} \boldsymbol{u})\left(\mathbf{I}+\mathbf{F}_{b}(\boldsymbol{u})\right) \mathbf{T} \boldsymbol{v} \\
& \cdot \mathbf{T} \delta \boldsymbol{u} \mathrm{d} \boldsymbol{x} \mathrm{d} t+\int_{0}^{T} \int_{\Gamma_{\mathrm{b}}} \delta C f(\mathbf{T} \boldsymbol{u}) \mathbf{T} \boldsymbol{u} \cdot \mathbf{T} \boldsymbol{v} \mathrm{d} \boldsymbol{x} \mathrm{d} t .
\end{aligned}
$$

With the forward solution $\left(\boldsymbol{u}^{*}, p^{*}, h^{*}\right)$ and the adjoint solution $\left(\boldsymbol{v}^{*}, q^{*}, \psi^{*}\right)$ satisfying (4) and (19), the first variation with respect to perturbations $\delta C$ in $C$ is (cf. A14)

$$
\delta \mathcal{L}=\int_{0}^{T} \int_{\Gamma_{\mathrm{b}}} f\left(\mathbf{T} \boldsymbol{u}^{*}\right) \mathbf{T} \boldsymbol{u}^{*} \cdot \mathbf{T} \boldsymbol{v}^{*} \delta C \mathrm{~d} \boldsymbol{x} \mathrm{d} t .
$$

\section{Appendix B: The adjoint solution in the inverse and sensitivity problems}

Assume that $\left(\boldsymbol{v}^{i}, q^{i}, \psi^{i}\right), i=1, \ldots, d$, solves adjoint FS equation (19) in the steady state with observation of $u_{i}$ with $d=2$ or 3

$\mathcal{F}=u_{i}(\boldsymbol{x})=\int_{\omega} u_{i} \delta(\boldsymbol{x}-\overline{\boldsymbol{x}}) \mathrm{d} \overline{\boldsymbol{x}}, F_{\boldsymbol{u}}=\boldsymbol{e}^{i} \delta(\boldsymbol{x}-\overline{\boldsymbol{x}})$,

$i=1, \ldots, d$,

or observation of $h$ with $d=1$

$\mathcal{F}=h(\boldsymbol{x})=\int_{\omega} h \delta(\boldsymbol{x}-\overline{\boldsymbol{x}}) \mathrm{d} \overline{\boldsymbol{x}}, F_{h}=\delta(\boldsymbol{x}-\overline{\boldsymbol{x}})$.

Introduce the weight functions $w_{i}(\boldsymbol{x}), i=1, \ldots, d$. It follows from (19) that $\left(w_{i}(\overline{\boldsymbol{x}}) \boldsymbol{v}^{i}(\boldsymbol{x}), w_{i}(\overline{\boldsymbol{x}}) q^{i}(\boldsymbol{x}), w_{i}(\overline{\boldsymbol{x}}) \psi^{i}(\boldsymbol{x})\right)$ is a solution with $F_{\boldsymbol{u}}=w_{i}(\overline{\boldsymbol{x}}) \boldsymbol{e}^{i} \delta(\boldsymbol{x}-\overline{\boldsymbol{x}})$ or $F_{h}=w(\overline{\boldsymbol{x}}) \delta(\boldsymbol{x}-\overline{\boldsymbol{x}})$. Therefore, also

$$
\left(\int_{\omega} w_{i}(\overline{\boldsymbol{x}}) \boldsymbol{v}^{i} \mathrm{~d} \overline{\boldsymbol{x}}, \int_{\omega} w_{i}(\overline{\boldsymbol{x}}) q^{i} \mathrm{~d} \overline{\boldsymbol{x}}, \int_{\omega} w_{i}(\overline{\boldsymbol{x}}) \psi^{i} \mathrm{~d} \overline{\boldsymbol{x}}\right)
$$

is a solution with $F_{\boldsymbol{u}}=\int_{\omega} w_{i}(\overline{\boldsymbol{x}}) \boldsymbol{e}^{i} \delta(\boldsymbol{x}-\overline{\boldsymbol{x}}) \mathrm{d} \overline{\boldsymbol{x}}=w_{i}(\boldsymbol{x}) \boldsymbol{e}^{i}$ or $F_{h}=\int_{\omega} w(\overline{\boldsymbol{x}}) \delta(\boldsymbol{x}-\overline{\boldsymbol{x}}) \mathrm{d} \overline{\boldsymbol{x}}=w(\boldsymbol{x})$. A sum over $i, i=1, \ldots, d$, of each integral in (B3) is also a solution. 
Consider a target functional $\mathcal{F}$ for the steady-state solution with a weight vector $\boldsymbol{w}(\overline{\boldsymbol{x}})$ with components $w_{i}(\overline{\boldsymbol{x}})$ multiplying $\delta u^{i}$ in the first variation of $\mathcal{F}$. Using (22), $\delta \mathcal{F}$ is

$$
\begin{aligned}
\delta \mathcal{F} & =\int_{\omega} \boldsymbol{w}(\overline{\boldsymbol{x}}) \cdot \delta \boldsymbol{u} \mathrm{d} \overline{\boldsymbol{x}} \\
& =\int_{\omega} \sum_{i=1}^{d} w_{i}(\overline{\boldsymbol{x}}) \delta u^{i} \mathrm{~d} \overline{\boldsymbol{x}} \\
& =\int_{\omega} \sum_{i=1}^{d} w_{i}(\overline{\boldsymbol{x}}) \int_{\Gamma_{\mathrm{b}}} f(\mathbf{T} \boldsymbol{u}) \mathbf{T} \boldsymbol{u} \cdot \mathbf{T} \boldsymbol{v}^{i} \delta C \mathrm{~d} \boldsymbol{x} \mathrm{d} \overline{\boldsymbol{x}} \\
& =\int_{\Gamma_{\mathrm{b}}} f(\mathbf{T} \boldsymbol{u}) \mathbf{T} \boldsymbol{u} \cdot \mathbf{T}\left(\int_{\omega} \sum_{i=1}^{d} w_{i}(\overline{\boldsymbol{x}}) \boldsymbol{v}^{i} \mathrm{~d} \overline{\boldsymbol{x}}\right) \delta C \mathrm{~d} \boldsymbol{x} .
\end{aligned}
$$

\section{Appendix C: Steady-state solution of the adjoint height equation in the FS model}

In a two-dimensional vertical ice with $\boldsymbol{u}(x, z)=\left(u_{1}, u_{3}\right)^{T}$, the stationary equation for $\psi$ in (19) is

$\left(u_{1} \psi\right)_{x}=F_{h}+\left(\boldsymbol{h} \psi+F_{\boldsymbol{u}}\right) \cdot \boldsymbol{u}_{z}, z=h, 0 \leq x \leq L$.

When $x>x_{*}$, where $F_{h}=0$ and $F_{\boldsymbol{u}}=0$, we have $\psi(x)=0$ since the right boundary condition is $\psi(L)=0$.

If $u_{1}$ is observed at $\Gamma_{\mathrm{s}}$ then $F(\boldsymbol{u}, h)=u_{1}(x) \chi(x), F_{\boldsymbol{u}}=$ $(\chi(x), 0)^{T}$, and $F_{h}=0$. The weight $\chi$ on $u_{1}$ may be a Dirac delta, a Gaussian, or a constant in a limited interval. On the other hand, if $F(\boldsymbol{u}, h)=h(x) \chi(x)$ then $F_{h}=\chi(x)$ and $F_{\boldsymbol{u}}=$ 0.

Let $g(x)=u_{1 z}(x)$ when $F_{\boldsymbol{u}} \neq \mathbf{0}$ and let $g(x)=1$ when $F_{h} \neq 0$. Then by (32),

$\left(u_{1} \psi\right)_{x}-\boldsymbol{h} \cdot \boldsymbol{u}_{z} \psi=g(x) \chi(x)$.

The solution to $(\mathrm{C} 2)$ is

$\psi(x)=-\frac{1}{u_{1}(x)} \int_{x}^{x_{*}} \exp \left(-\int_{x}^{\xi} \frac{\boldsymbol{h} \cdot \boldsymbol{u}_{z}(y)}{u_{1}(y)} \mathrm{d} y\right) g(\xi) \chi(\xi) \mathrm{d} \xi$,

$0 \leq x<x_{*}, \psi(x)=0, x_{*}<x \leq L$.

In particular, if $\chi(x)=\delta\left(x-x_{*}\right)$ then $\mathcal{F}=u_{1}\left(x_{*}\right)$ or $\mathcal{F}=$ $h\left(x_{*}\right)$, and the multiplier is

$\psi(x)=-\frac{g\left(x_{*}\right)}{u_{1}(x)} \exp \left(-\int_{x}^{x_{*}} \frac{\boldsymbol{h} \cdot \boldsymbol{u}_{z}(y)}{u_{1}(y)} \mathrm{d} y\right)$,

$0 \leq x<x_{*}$,

which has a jump $-g\left(x_{*}\right) / u_{1}\left(x_{*}\right)$ at $x_{*}$.

\section{Appendix D: Simplified SSA equations}

The forward and adjoint SSA equations in (12) and (40) are solved analytically. The conclusion from the thickness equation in (12) is that

$u(x) H(x)=u(0) H(0)+a x=a x$,

since $u(0)=0$. Solve the second equation in (12) for $u$ on the bedrock with $x \leq x_{\mathrm{GL}}$ and insert into (D1) using the assumptions for $x>0$ that $b_{x} \ll H_{x}$ and $h_{x} \approx H_{x}$ to have

$\frac{\rho g}{C} H^{m+1} H_{x}=\frac{\rho g}{C(m+2)}\left(H^{m+2}\right)_{x}=-(a x)^{m}$.

The equation for $H^{m+2}$ for $x \leq x_{\mathrm{GL}}$ is integrated from $x$ to $x_{\mathrm{GL}}$ such that

$$
\begin{aligned}
& H(x)=\left(H_{\mathrm{GL}}^{m+2}+\frac{m+2}{m+1} \frac{C a^{m}}{\rho g}\left(x_{\mathrm{GL}}^{m+1}-x^{m+1}\right)\right)^{\frac{1}{m+2}}, \\
& u(x)=\frac{a x}{H}, \quad H_{x}=-\frac{C a^{m}}{\rho g} \frac{x^{m}}{H^{m+1}} .
\end{aligned}
$$

For the floating ice at $x>x_{\mathrm{GL}}, \rho g H h_{x}=0$, implying that $h_{x}=0$ and $H_{x}=0$. Hence, $H(x)=H_{\mathrm{GL}}$. The velocity increases linearly beyond the grounding line

$u(x)=a x / H(x)=a x / H_{\mathrm{GL}}, x>x_{\mathrm{GL}}$.

By including the viscosity term in (11) and assuming that $H(x)$ is linear in $x$, a more accurate formula is obtained for $u(x)$ on the floating ice in (6.77) of Greve and Blatter (2009).

\section{Appendix E: Jumps in $\psi$ and $v$ in SSA}

Multiply the first equation in (40) by $H$ and the second equation by $u$ to eliminate $\psi_{x}$. We get

$-C m u^{m} v-\rho g H^{2} v_{x}=H F_{h}-u F_{\mathrm{u}}$.

Use the expression for $u$ and $H_{x}$ in (D3). Then

$\rho g H\left(m H_{x} v-H v_{x}\right)=H F_{h}-u F_{\mathrm{u}}$,

or equivalently

$\left(\frac{v}{H^{m}}\right)_{x}=-\frac{1}{\rho g H^{m+2}}\left(H F_{h}-u F_{\mathrm{u}}\right)$.

The solutions $\psi(x)$ and $v(x)$ of the adjoint SSA equation (37) have jumps at the observation point $x_{*}$. For $x$ close to $x_{*}$ in a short interval $\left[x_{*}^{-}, x_{*}^{+}\right]$with $x_{*}^{-}<x_{*}<x_{*}^{+}$, integrate (E3) to receive

$\int_{x_{*}^{-}}^{x_{*}^{+}}\left(\frac{v}{H^{m}}\right)_{x} \mathrm{~d} x=-\int_{x_{*}^{-}}^{x_{*}^{+}} \frac{H F_{h}-u F_{\mathrm{u}}}{\rho g H^{m+2}} \mathrm{~d} x$. 
Since $H$ is continuous and $u$ and $v$ are bounded, when $x_{*}^{-} \rightarrow$ $x_{*}^{+}$, then

$$
\begin{aligned}
& v\left(x_{*}^{+}\right)-v\left(x_{*}^{-}\right)= \\
& -\frac{1}{\rho g H_{*}^{2}}\left(H_{*} \int_{x_{*}^{-}}^{x_{*}^{+}} F_{h} \mathrm{~d} x-u_{*} \int_{x_{*}^{-}}^{x_{*}^{+}} F_{\mathrm{u}} \mathrm{d} x\right) .
\end{aligned}
$$

A similar relation for $\psi$ can be derived:

$$
\psi\left(x_{*}^{+}\right)-\psi\left(x_{*}^{-}\right)=\frac{1}{H_{*}} \int_{x_{*}^{-}}^{x_{*}^{+}} F_{\mathrm{u}} \mathrm{d} x .
$$

With $F_{\mathrm{u}}=0$ and $F_{h}=0$ for $x<x_{*}$ and $v(0)=\psi_{x}(0)=0$, we find that

$v(x)=\psi_{x}(x)=0, \psi(x)=\psi\left(x_{*}^{-}\right), 0 \leq x<x_{*}$.

If $F(u, h)=u \delta\left(x-x_{*}\right)$, then by (E5) and (E6)

$v\left(x_{*}^{+}\right)=\frac{u_{*}}{\rho g H_{*}^{2}}, \psi\left(x_{*}^{+}\right)-\psi\left(x_{*}^{-}\right)=\frac{1}{H_{*}}$,

and if $F(u, h)=h \delta\left(x-x_{*}\right)$, then

$v\left(x_{*}^{+}\right)=-\frac{1}{\rho g H_{*}}, \psi\left(x_{*}^{+}\right)-\psi\left(x_{*}^{-}\right)=0$.

\section{Appendix F: Analytical solutions in SSA}

By Appendix E, $v(x)=0$ for $0 \leq x<x_{*}$. Use equations in (40) with $H_{x}$ in (D3) for $x_{*}<x \leq x_{\mathrm{GL}}$ to have

$\frac{v_{x}}{v}=-\frac{a x C m u^{m-1}}{\rho g H^{3}}=-\frac{C m u^{m}}{\rho g H^{2}}=\frac{m H_{x}}{H}$.

Let $\mathcal{H}\left(x-x_{*}\right)=\int_{-\infty}^{x-x_{*}} \delta(s) d s$ be the Heaviside step function at $x_{*}$. Then

$v(x)=C_{v} H(x)^{m} \mathcal{H}\left(x-x_{*}\right), 0 \leq x \leq x_{\mathrm{GL}}$.

To satisfy the jump condition in (E8) and (E9), the constant $C_{v}$ is

$C_{v}= \begin{cases}\frac{a x_{*}}{\rho g H_{*}^{m+3}}, & F(u, h)=u \delta\left(x-x_{*}\right), \\ -\frac{1}{\rho g H_{*}^{m+1}}, & F(u, h)=h \delta\left(x-x_{*}\right) .\end{cases}$

Combine (F1) with the relation $\psi_{x}=\left(F_{\mathrm{u}}-C m u^{m-1} v\right) / H$ and integrate from $x$ to $x_{\mathrm{GL}}$ to obtain

$\psi(x)=C_{v} a^{m-1} C\left(x_{\mathrm{GL}}^{m}-x^{m}\right), x_{*}<x \leq x_{\mathrm{GL}}$.
With the jump condition in (E8) and (E9), $\psi(x)$ at $0 \leq x<$ $x_{*}$ is

$\psi(x)=\left\{\begin{array}{l}-\frac{1}{H_{*}}+\frac{C a^{m} x_{*}}{\rho g H_{*}^{m+3}}\left(x_{\mathrm{GL}}^{m}-x_{*}^{m}\right), \\ F(u, h)=u \delta\left(x-x_{*}\right), \\ -\frac{C a^{m-1}}{\rho g H_{*}^{m+1}}\left(x_{\mathrm{GL}}^{m}-x_{*}^{m}\right), \\ F(u, h)=h \delta\left(x-x_{*}\right) .\end{array}\right.$

The weight for $\delta C$ in the functional $\delta \mathcal{L}$ in (38) is non-zero for $x_{*}<x \leq x_{\mathrm{GL}}$ :

$-v u^{m}=-C_{v}(a x)^{m}$.

Use (F1) and (40) in (38) to determine the weight for $\delta b$ in $\delta \mathcal{L}$

$\psi_{x} u+v_{x} \eta u_{x}+v \rho g h_{x}=\rho g(H v)_{x}+F_{h}$

$=C_{v} \rho g H^{m}\left[(m+1) H_{x} \mathcal{H}\left(x-x_{*}\right)+H \delta\left(x-x_{*}\right)\right]$

$+F_{h}$. 
Code availability. The FS equations are solved using Elmer/Ice version 8.4 (rev. f6bfdc9) with the scripts at https://doi.org/10.5281/zenodo.3611158 (Cheng, 2020a). The forward and adjoint SSA solvers are implemented in MATLAB. The code is available at https://doi.org/10.5281/zenodo.3611154 (Cheng, 2020b).

Author contributions. GC and PL designed the study. GC did the numerical computations. GC, PL, and NK discussed and wrote the manuscript.

Competing interests. The authors declare that they have no conflict of interest.

Acknowledgements. We are grateful to Lina von Sydow for reading a draft of the paper and helping us improve the presentation with her comments. We would also like to thank the editor Olivier Gagliardini and the two anonymous reviewers.

Financial support. This research has been supported by a Svenska Forskningsrådet Formas grant (no. 2017-00665) to Nina Kirchner and the Swedish strategic research program eSSENCE.

Review statement. This paper was edited by Olivier Gagliardini and reviewed by two anonymous referees.

\section{References}

Ahlkrona, J., Lötstedt, P., Kirchner, N., and Zwinger, T.: Dynamically coupling the non-linear Stokes equstions with the shallow ice approximation in glaciology: Description and first applications of the ISCAL method, J. Comput. Phys., 308, 1-19, 2016.

Brondex, J., Gagliardini, O., Gillet-Chaulet, F., and Durand, G.: Sensitivity of grounding line dynamics to the choice of the friction law, J. Glaciology, 63, 854-866, 2017.

Brondex, J., Gillet-Chaulet, F., and Gagliardini, O.: Sensitivity of centennial mass loss projections of the Amundsen basin to the friction law, The Cryosphere, 13, 177-195, https://doi.org/10.5194/tc-13-177-2019, 2019.

Cheng, G.: Numerical experiments for FS adjoint, Zenodo, https://doi.org/10.5281/zenodo.3611158, 2020a.

Cheng, G.: Numerical experiments for SSA adjoint, Zenodo, https://doi.org/10.5281/zenodo.3611154, 2020b.

Cheng, G. and Lötstedt, P.: Parameter sensitivity analysis of dynamic ice sheet models - numerical computations, The Cryosphere, 14, 673-691, https://doi.org/10.5194/tc-14-6732020, 2020.

Farinotti, D., Longuevergne, L., Moholdt, G., Duethmann, D., Mölg, T., Bolch, T., Vorogushyn, S., and Güntner, A.: Substantial glacier mass loss in the Tien Shan over the past 50 years, Nat. Geosci., 8, 716-722, https://doi.org/10.1038/ngeo2513, 2015.
Fisher, A. T., Mankoff, K. D., Tulaczyk, S. M., Tyler, S. W., and Foley, N.: High geothermal heat flux measured below the West Antarctic Ice Sheet, Science Advances, 1, e1500093, https://doi.org/10.1126/sciadv.1500093, 2015.

Fowler, A. C.: Weertman, Lliboutry and the development of sliding theory, J. Glaciol., 56, 965-972, https://doi.org/10.3189/002214311796406112, 2011.

Gagliardini, O., Zwinger, T., Gillet-Chaulet, F., Durand, G., Favier, L., de Fleurian, B., Greve, R., Malinen, M., Martín, C., Råback, P., Ruokolainen, J., Sacchettini, M., Schäfer, M., Seddik, H., and Thies, J.: Capabilities and performance of Elmer/Ice, a newgeneration ice sheet model, Geosci. Model Dev., 6, 1299-1318, https://doi.org/10.5194/gmd-6-1299-2013, 2013.

Gillet-Chaulet, F., Durand, G., Gagliardini, O., Mosbeux, C., Mouginot, J., Rémy, F., and Ritz, C.: Assimilation of surface velocities acquired between 1996 and 2010 to constrain the form of the basal friction law under Pine Island Glacier, Geophys. Res. Lett., 43, 10311-10321, 2016.

Gladstone, R. M., Warner, R. C., Galton-Fenzi, B. K., Gagliardini, O., Zwinger, T., and Greve, R.: Marine ice sheet model performance depends on basal sliding physics and sub-shelf melting, The Cryosphere, 11, 319-329, https://doi.org/10.5194/tc-11319-2017, 2017.

Glen, J. W.: The creep of polycrystalline ice, P. Roy. Soc. Lond. A Mat., 228, 519-538, 1955.

Goldberg, D. N., Heimbach, P., Joughin, I., and Smith, B.: Committed retreat of Smith, Pope, and Kohler Glaciers over the next 30 years inferred by transient model calibration, The Cryosphere, 9, 2429-2446, https://doi.org/10.5194/tc-9-2429-2015, 2015.

Greve, R. and Blatter, H.: Dynamics of Ice Sheets and Glaciers, Advances in Geophysical and Environmental Mechanics and Mathematics (AGEM ${ }^{2}$ ), Springer, Berlin, 287 pp., https://doi.org/10.1007/978-3-642-03415-2, 2009.

Gudmundsson, G. H.: Transmission of basal variability to glacier surface, J. Geophys. Res., 108, 2253, https://doi.org/10.1029/2002JB002107, 2003.

Gudmundsson, G. H.: Analytical solutions for the surface response to small amplitude perturbations in boundary data in the shallow-ice-stream approximation, The Cryosphere, 2, 77-93, https://doi.org/10.5194/tc-2-77-2008, 2008.

Gudmundsson, G. H. and Raymond, M.: On the limit to resolution and information on basal properties obtainable from surface data on ice streams, The Cryosphere, 2, 167-178, https://doi.org/10.5194/tc-2-167-2008, 2008.

Heimbach, P. and Losch, M.: Adjoint sensitivities of sub-ice-shelf melt rates to ocean circulation under the Pine Island Ice Shelf, West Antarctica, Ann. Glaciol., 53, 59-69, 2012.

Hutter, K.: Theoretical Glaciology, D. Reidel Publishing Company, Terra Scientific Publishing Company, Dordrecht, the Netherlands, https://doi.org/10.1007/978-94-015-1167-4, 510 pp., 1983.

Iken, A.: The effect of the subglacial water pressure on the sliding velocity of a glacier in an idealized numerical model, J. Glaciol., 27, 407-421, https://doi.org/10.1017/S0022143000011448, 1981.

Isaac, T., Petra, N., Stadler, G., and Ghattas, O.: Scalable and efficient algorithms for the propagation of uncertainty from data through inference to prediction for large-scale problems with ap- 
plication to flow of the Antarctic ice sheet, J. Comput. Phys., 296, 348-368, 2015

Jay-Allemand, M., Gillet-Chaulet, F., Gagliardini, O., and Nodet, M.: Investigating changes in basal conditions of Variegated Glacier prior to and during its 1982-1983 surge, The Cryosphere, 5, 659-672, https://doi.org/10.5194/tc-5-659-2011, 2011.

Key, K. and Siegfried, M. R.: The feasibility of imaging subglacial hydrology beneath ice streams with ground-based electromagnetics, J. Glaciol., 63, 755-771, https://doi.org/10.1017/jog.2017.36, 2017.

Kyrke-Smith, T. M., Gudmundsson, G. H., and Farrell, P. E.: Relevance of detail in basal topography for basal slipperiness inversions: a case study on Pine Island Glacier, Antarctica, Frontiers Earth Sci., 6, 33, 2018.

Lliboutry, L.: General Theory of Subglacial Cavitation and Sliding of Temperate Glaciers, J. Glaciol., 7, 21-58, https://doi.org/10.3189/s0022143000020396, 1968.

MacAyeal, D. R.: Large-scale ice flow over a viscous basal sediment: Theory and application to Ice Stream B, Antarctica., J. Geophys. Res., 94, 4071-4078, 1989.

MacAyeal, D. R.: A tutorial on the use of control methods in ice sheet modeling, J. Glaciol., 39, 91-98, 1993.

Maier, N., Humphrey, N., Harper, J., and Meierbachtol, T.: Sliding dominates slow-flowing margin regions, Greenland Ice Sheet, Science Advances, 5, eaaw5406, https://doi.org/10.1126/sciadv.aaw5406, 2019.

Martin, N. and Monnier, J.: Adjoint accuracy for the full Stokes ice flow model: limits to the transmission of basal friction variability to the surface, The Cryosphere, 8, 721-741, https://doi.org/10.5194/tc-8-721-2014, 2014.

Minchew, B., Simons, M., Björnsson, H., Pálsson, F., Morlighem, M., Seroussi, H., Larour, E., and Hensley, S.: Plastic bed beneath Hofsjökull Ice Cap, central Iceland, and the sensitivity of ice flow to surface meltwater flux, J. Glaciol., 62, 147-158, 2016.

Minchew, B. M., Meyer, C. R., Pegler, S. S., Lipovsky, B. P., Rempel, A. W., Gudmundsson, G. H., and Iverson, N. R.: Comment on "Friction at the bed does not control fast glacier flow", Science, 363, eaau6055, https://doi.org/10.1126/science.aau6055, 2019

Monnier, J. and des Boscs, P.-E.: Inference of the bottom properties in shallow ice approximation models, Inverse Probl., 33, 115001, https://doi.org/10.1088/1361-6420/aa7b92, 2017.

Morland, L. W.: Unconfined Ice-Shelf Flow, in: Dynamics of the West Antarctic Ice Sheet, edited by: van der Veen, C. J. and Oerlemans, J., Springer, Netherlands, 99-116, 1987.

Morlighem, M., Seroussi, H., Larour, E., and Rignot, E.: Inversion of basal friction in Antarctica using exact and incomplete adjoints of a high-order model, J. Geophys. Res.-Earth Surf., 118, $1-8,2013$.

Mouginot, J., Rignot, E., Bjørk, A. A., van den Broeke, M., Millan, R., Morlighem, M., Noël, B., Scheuchl, B., and Wood, M.: Forty-six years of Greenland Ice Sheet mass balance from 1972 to 2018, P. Natl. Acad. Sci. USA, 116, 9239-9244, https://doi.org/10.1073/pnas.1904242116, 2019.

Nye, J. F.: The motion of ice sheets and glaciers, J. Glaciol., 3, 493507,1959

Nye, J. F.: A calculation on the sliding of ice over a wavy surface using a Newtonian viscous approximation, P. R. Soc. A, 311, 445467, 1969.
Pattyn, F. and Morlighem, M.: The uncertain future of the Antarctic Ice Sheet, Science, 367, 1331-1335, 2020.

Pattyn, F., Schoof, C., Perichon, L., Hindmarsh, R. C. A., Bueler, E., de Fleurian, B., Durand, G., Gagliardini, O., Gladstone, R., Goldberg, D., Gudmundsson, G. H., Huybrechts, P., Lee, V., Nick, F. M., Payne, A. J., Pollard, D., Rybak, O., Saito, F., and Vieli, A.: Results of the Marine Ice Sheet Model Intercomparison Project, MISMIP, The Cryosphere, 6, 573-588, https://doi.org/10.5194/tc-6-573-2012, 2012.

Perego, M., Price, S. F., and Stadler, G.: Optimal initial conditions for coupling ice sheet models to Earth system models, J. Geophys. Res.-Earth Surf., 119, 1894-1917, 2014.

Petra, N., Zhu, H., Stadler, G., Hughes, T. J. R., and Ghattas, O.: An inexact Gauss-Newton method for inversion of basal sliding and rheology parameters in a nonlinear Stokes ice sheet model, J. Glaciol., 58, 889-903, 2012.

Petra, N., Martin, J., Stadler, G., and Ghattas, O.: A computational framework for infinite-dimensional Bayesian inverse problems, Part II: Newton MCMC with application to ice sheet flow inverse problems, SIAM J. Sci. Comput., 36, A1525-A1555, 2014.

Pörtner, H.-O., Roberts, D. C., Masson-Delmotte, V., Zhai, P., Poloczanska, E., Mintenbeck, K., Tignor, M., Alegría, A., Nicolai, M., Okem, A., Petzold, J., Rama, B., and Weyer, N. M.: IPCC, 2019: Technical Summary, in: IPCC Special Report on the Ocean and Cryosphere in a Changing Climate, in press, 2019.

Rignot, E., Mouginot, J., Scheuchl, B., van den Broeke, M., van Wessem, M. J., and Morlighem, M.: Four decades of Antarctic ice sheet mass balance from 1979-2017, P. Natl. Acad. Sci. USA, 116, 1095-1103, https://doi.org/10.1073/pnas.1812883116, 2019.

Ritz, C., Edwards, T. L., Durand, G., Payne, A. J., Peyaud, V., and Hindmarsh, R. C.: Potential sea level rise from Antarctic icesheet instability constrained by observations, Nature, 528, 115$118,2015$.

Schannwell, C., Drews, R., Ehlers, T. A., Eisen, O., Mayer, C., and Gillet-Chaulet, F.: Kinematic response of ice-rise divides to changes in ocean and atmosphere forcing, The Cryosphere, 13, 2673-2691, https://doi.org/10.5194/tc-13-2673-2019, 2019.

Schoof, C.: The effect of cavitation on glacier sliding, P. R. Soc. A, 461, 609-627, 2005.

Schoof, C.: Ice sheet grounding line dynamics: Steady states, stability and hysteresis, J. Geophys. Res.-Earth Surf., 112, F03S28, https://doi.org/10.1029/2006JF000664, 2007.

Schoof, C.: Ice-sheet acceleration driven by melt supply variability, Nature, 468, 803-806, 2010.

Sergienko, O. and Hindmarsh, R. C. A.: Regular patterns in frictional resistance of ice-stream beds seen by surface data inversion, Science, 342, 1086-1089, 2013.

Seroussi, H., Morlighem, M., Larour, E., Rignot, E., and Khazendar, A.: Hydrostatic grounding line parameterization in ice sheet models, The Cryosphere, 8, 2075-2087, https://doi.org/10.5194/tc-8-2075-2014, 2014.

Smith, R. C.: Uncertainty Quantification: Theory, Implementation, and Applications, Society for Industrial and Applied Mathematics, Philadelphia, ISBN 9781611973211, 382 pp., 2014.

Sole, A. J., Mair, D. W. F., Nienow, P. W., Bartholomew, I. D., King, I. D., Burke, M. A., and Joughin, I.: Seasonal speedup of a Greenland marine-terminating outlet glacier forced by surface 
melt-induced changes in subglacial hydrology, J. Geophys. Res., 116, F03014, https://doi.org/10.1029/2010JF001948, 2011.

Stearn, L. A. and van der Veen, C. J.: Friction at the bed does not control fast glacier flow, Science, 361, 273-277, 2018.

Steffen, W., Rockström, J., Richardson, K., Lenton, T. M., Folke, C., Liverman, D., Summerhayes, C. P., Barnosky, A. D., Cornell, S. E., Crucifix, M., Donges, J. F., Fetzer, I., Lade, S. J., Scheffer, M., Winkelmann, R., and Schellnhuber, H. J.: Trajectories of the Earth System in the Anthropocene, P. Natl. Acad. Sci. USA, 115, 8252-8259, https://doi.org/10.1073/pnas.1810141115, 2018.

Thorsteinsson, T., Raymond, C. F., Gudmundsson, G. H., Bindschadler, R. A., Vornberger, P., and Joughin, I.: Bed topography and lubrication inferred from surface measurements on fastflowing ice streams, J. Glaciol., 49, 481-490, 2003.

Tsai, V. C., Stewart, A. L., and Thompson, A. F.: Marine ice-sheet profiles and stability under Coulomb basal conditions, J. Glaciol., 61, 205-215, 2015.

Vallot, D., Pettersson, R., Luckman, A., Benn, D. I., Zwinger, T., van Pelt, W. J. J., Kohler, J., Schäfer, M., Claremar, B., and Hulton, N. R. J.: Basal dynamics of Kronebreen, a fast-flowing tidewater glacier in Svalbard: non-local spatio-temporal response to water input, J. Glaciol., 11, 179-190, 2017.

van der Veen, C. J.: Tidewater calving, J. Glaciol., 42, 375-385, 1996.

van Pelt, W. J. J., Oerlemans, J., Reijmer, C. H., Pettersson, R., Pohjola, V. A., Isaksson, E., and Divine, D.: An iterative inverse method to estimate basal topography and initialize ice flow models, The Cryosphere, 7, 987-1006, https://doi.org/10.5194/tc-7987-2013, 2013. van Vuuren, D. P., Edmonds, J., Kainuma, M., Riahi, K., Thomson, A., Hibbard, K., Hurtt, G. C., Kram, T., Krey, V., Lamarque, J.F., Masui, T., Meinshausen, M., Nakicenovic, N., Smith, S. J., and Rose, S. K.: The representative concentration pathways: an overview, Climatic Change, 109, 5, 2011.

Weertman, J.: On the sliding of glaciers, J. Glaciol., 3, 33-38, 1957.

Weertman, J.: Equilibrium profile of ice caps, J. Glaciol., 3, 953964, 1961.

Wilkens, N., Behrens, J., Kleiner, T., Rippin, D., Rückamp, M., and Humbert, A.: Thermal structure and basal sliding parametrisation at Pine Island Glacier - a 3D full-Stokes model study, The Cryosphere, 9, 675-690, https://doi.org/10.5194/tc-9-675-2015, 2015.

Yu, H., Rignot, E., Seroussi, H., and Morlighem, M.: Retreat of Thwaites Glacier, West Antarctica, over the next 100 years using various ice flow models, ice shelf melt scenarios and basal friction laws, The Cryosphere, 12, 3861-3876, https://doi.org/10.5194/tc-12-3861-2018, 2018.

Zhu, H., Petra, N., Stadler, G., Isaac, T., Hughes, T. J. R., and Ghattas, O.: Inversion of geothermal heat flux in a thermomechanically coupled nonlinear Stokes ice sheet model, The Cryosphere, 10, 1477-1494, https://doi.org/10.5194/tc-10-1477-2016, 2016.

Zoet, L. K. and Iverson, N. R.: A slip law for glaciers on deformable beds, Science, 368, 76-78, 2020. 